\title{
In vivo modulation of leukocyte-endothelium interactions
}

Citation for published version (APA):

Tromp, S. C. (1999). In vivo modulation of leukocyte-endothelium interactions. [Doctoral Thesis, Maastricht University]. Universiteit Maastricht. https://doi.org/10.26481/dis.19991203st

Document status and date:

Published: 01/01/1999

DOI:

10.26481/dis.19991203st

Document Version:

Publisher's PDF, also known as Version of record

\section{Please check the document version of this publication:}

- A submitted manuscript is the version of the article upon submission and before peer-review. There can be important differences between the submitted version and the official published version of record.

People interested in the research are advised to contact the author for the final version of the publication, or visit the DOI to the publisher's website.

- The final author version and the galley proof are versions of the publication after peer review.

- The final published version features the final layout of the paper including the volume, issue and page numbers.

Link to publication

\footnotetext{
General rights rights.

- You may freely distribute the URL identifying the publication in the public portal. please follow below link for the End User Agreement:

www.umlib.nl/taverne-license

Take down policy

If you believe that this document breaches copyright please contact us at:

repository@maastrichtuniversity.nl

providing details and we will investigate your claim.
}

Copyright and moral rights for the publications made accessible in the public portal are retained by the authors and/or other copyright owners and it is a condition of accessing publications that users recognise and abide by the legal requirements associated with these

- Users may download and print one copy of any publication from the public portal for the purpose of private study or research.

- You may not further distribute the material or use it for any profit-making activity or commercial gain

If the publication is distributed under the terms of Article $25 \mathrm{fa}$ of the Dutch Copyright Act, indicated by the "Taverne" license above, 


\section{IN VIVO MODULATION OF \\ LEUKOCYTE-ENDOTHELIUM INTERACTIONS}


(C) S.C. Tromp, Maastricht 1999

ISBN 90-9013280-5

Druk: Datawyse| Universitaire Pers Maastricht 


\section{IN VIVO MODULATION OF LEUKOCYTE-ENDOTHELIUM INTERACTIONS}

\section{Proefschrift}

ter verkrijging van de graad van doctor aan de Universiteit Maastricht, op gezag van de Rector Magnificus, Prof. Dr. A.C. Nieuwenhuijzen Kruseman, volgens het besluit van het College van Dekanen in het openbaar te verdedigen op vrijdag 3 december 1999 om 14.00 uur

door

Selma Corine Tromp

geboren op 6 april 1971 te Hellendoorn 


\section{Promotores:}

Prof. Dr. R.S. Reneman

Prof. Dr. G.J. Tangelder (Vrije Universiteit, Amsterdam)

Prof. Dr. D.W. Slaaf

\section{Co-promotor:}

Dr. M.G.A. oude Egbrink

\section{Beoordelingscommissie:}

Prof. Dr. H.A.J. Struijker Boudier (voorzitter)

Prof. Dr. J.W. Arends

Prof. Dr. P.J.E.H.M. Kitslaar

Prof. Dr. D. Vestweber (Universität Münster, Duitsland)

Prof. Dr. G.J. van der Vusse 
"Tevreden liet hij zich achteroverzakken, nam een patriarchale houding aan, en wijdde zich sterk zwetend aan de polynucleaire leucocyten, deze geheimzinnige en nijvere wezentjes, die zoveel in het menselijk lichaam op te knappen hadden."

Simon Vestdijk - De vrije vogel en zijn kooien

"Men doet er niets aan af, men voegt er niets aan toe: de wonderdaden van de Heer zijn niet te doorgronden."

Wijsheid van Jezus Sirach 18:6 



\section{CONTENTS}

\section{GENERAL INTRODUCTION}

1. Introduction 11

2. Leukocyte-endothelium interactions; a literature survey 15

2.1 Leukocytes 15

2.2.1 Development and differentiation 15

2.2.2 Functions 16

2.2 Endothelium 18

2.3 Leukocyte-endothelium interactions 18

$\begin{array}{ll}2.3 .1 & \text { Leukocyte margination } \\ & 19\end{array}$

2.3.2 Leukocyte rolling 20

2.3.3 Leukocyte activation 24

2.3.4 Leukocyte adhesion 25

2.3.5 Diapedesis and migration 26

2.3.6 Sequential contribution of adhesion molecules 27

2.3.7 In vitro versus in vivo 27

2.3.8 Venules versus arterioles 29

2.3.9 Leukocyte adhesion deficiency 29

2.4 Modulation of leukocyte-endothelium interactions 30

3. Methodological survey 37

3.1 Animals and experimental setup 37

3.2 Assessment of microvascular hemodynamic parameters 39

3.3 Evaluation of leukocyte-endothelium interactions 41

3.4 Statistics and data presentation 45

4. How to administer agents to study their local effect on leukocyte-endothelium interactions in mesenteric venules 49

4.1 Introduction 50

4.2 Materials and methods $\quad 50$

4.3 Results 52

4.4 Discussion 55

\section{INDUCTION OF LEUKOCYTE-ENDOTHELIUM INTERACTIONS 57}

5. Are mast cells involved in regulation of leukocyte rolling in rabbit mesenteric venules?

5.1 Introduction 60

5.2 Materials and methods 60

5.3 Results and discussion 61 
6. The role of mast cells and histamine in leukocyte-endothelium interactions in four rat strains

6.1 Introduction 66

6.2 Materials and methods 66

6.3 Results 69

6.4 Discussion 76

INHIBITION OF LEUKOCYTE-ENDOTHELIUM INTERACTIONS $\quad 79$

7. The role of selectins in the thromboembolism induced reduction in leukocyte rolling $\quad 81$

$\begin{array}{lll}7.1 & \text { Introduction } & 82\end{array}$

$\begin{array}{ll}7.2 & \text { Materials and methods } \\ 7.32\end{array}$

$\begin{array}{lll}7.3 & \text { Results } & 86\end{array}$

7.4 Discussion 90

8. The influence of prostaglandins on leukocyte-endothelium interactions 93

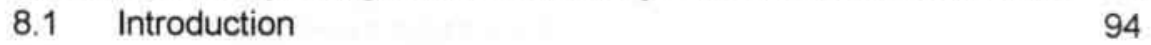

8.2 Materials and methods 95

$\begin{array}{lll}8.3 & \text { Results } & 97\end{array}$

8.4 Discussion 100

9. Tumor angiogenesis factors reduce leukocyte adhesion in vivo 105

9.1 Introduction 106

$\begin{array}{lll}9.2 & \text { Materials and methods } & 107\end{array}$

9.3 Results 109

9.4 Discussion 111

CONCLUSIONS 115

10. General discussion 117

References 123

$\begin{array}{ll}\text { Summary } & 137\end{array}$

Samenvatting 141

List of abbreviations $\quad 147$

$\begin{array}{ll}\text { Publications } & 149\end{array}$

Curriculum vitae $\quad 151$

Dankwoord 153 

The major function of white blood cells (leukocytes) is to protect the body against 'foreign' invaders, like infectious bacteria, viruses, and toxic agents. To be able to exert this function leukocytes have to be available throughout the body. To this purpose leukocytes move from their site of formation (bone marrow and lymph tissue) into the vascular system. When passing an inflamed area, the leukocytes have to move through the vessel wall into the surrounding tissue. This process, called diapedesis or extravasation can be observed with the use of intravital microscopy and has been described as one of the first by Cohnheim (1839-1884) in fascinating detail:

" $\{$ In the veins of the frog mesentery\} a very characteristic condition immediately begins to develop, slowly, under the eye of the observer. The peripheral zone of the blood stream, the original plasma layer, becomes filled with innumerable colorless blood corpuscles. $\{\ldots\}$ At the outer border of the wall of the vein there arise a few small colorless button-shaped elevations $\{\ldots\}$. These excrescences slowly and very gradually become larger. $\{\ldots\}$ Finally $\{$ it $\}$ becomes separated from the point on the wall where it had been attached and we now have before us a colorless somewhat shiny contractile corpuscle $\{\ldots\}$. The size of the corpuscle corresponds completely with that of a white blood corpuscle. $\{\ldots\}$ Consequently it is in no way distinguishable from a colorless blood corpuscle." (Cohnheim cited in Jarcho, 1972)

In the next decades the different steps between leukocytes freely flowing in the blood stream and leukocyte migration into the tissue have been unravelled. It appears to be a sequence of steps, in which each step has to be completed for the next step to occur (Butcher, 1991; von Andrian et al., 1991; Springer, 1994). First, leukocytes move in the venular blood stream towards the vessel wall (margination). When the endothelial cells of the vessel wall are stimulated to express adhesion molecules, leukocytes are able to start to roll along the endothelial cell layer at a significantly lower rate than the velocity of the free flowing blood cells. Due to their presence near the vessel wall they can become activated by substances released by vascular cells. Subsequently, activated leukocytes can adhere to the endothelial cells and move through the vessel wall into the surrounding inflamed tissue. Various adhesion molecules on leukocytes and on endothelial cells that mediate different steps in the cascade have been identified (Springer, 1994; Gahmberg et al., 1997; Vestweber and Blanks, 1999). It is highly probable that more adhesion molecules as well as their ligands are still to be discovered.

In the last decades, it became clear that the basis for diseases such as arthritis, asthma, or psoriasis is an overactivity of leukocytes which may lead to an attack against the host itself (Lasky, 1992; Brown, 1997). Inhibition of leukocyteendothelium interactions might have therapeutic potential in such diseases. Therefore, many studies have focussed on the mechanisms behind the normal induction of adhesion molecule expression and, hence, leukocyte-endothelium 
interactions or on ways to modulate these. Most of these studies have been performed in vitro, with the aim to control the experimental conditions, and to test effects of changes in one variable. However, data obtained in vitro are not necessarily representative of the in vivo situation, in which factors like fluid dynamic conditions, blood contents, and various agents, originating from the inflamed tissue, the leukocytes, or the vessel wall, influence the process under study.

The studies described in the present thesis aim to provide more insight into the effect(s) of agents from cells of the vessel wall and/or the surrounding tissue on leukocyte-endothelium interactions in vivo. To this purpose, leukocyte-endothelium interactions, in particular leukocyte rolling and adhesion, were studied with the help of intravital videomicroscopy. The effects of various interventions were quantified. In this way, we investigated the involvement of several agents in the induction and the inhibition of leukocyte-endothelium interactions.

The thesis is structured as follows. The first part of the thesis is a general introduction. In chapter $\mathbf{2}$ a brief overview is given of literature on leukocyteendothelium interactions and of present knowledge of the way these interactions can be induced or inhibited by environmental factors. Emphasis will be on studies performed in vivo. Chapter 3 provides information about the animals and the experimental setups used in the various studies. Furthermore, in this chapter an explanation of and motivation for the experimental parameters used in the studies is given as well as an evaluation of the way data are presented and statistically evaluated. One of the aims of the present thesis is to investigate local effects of agents on leukocyte-endothelium interactions. Therefore, we studied with the use of a frequently used vehicle, i.e., saline, the best route of administration of various agents (chapter 4). To this purpose we compared the effects of local intravascular administration of saline with those of topical application.

The second part of the thesis consists of two studies on the induction of leukocyte-endothelium interactions, more in particular on the involvement of mast cells in the induction of leukocyte rolling in rabbits (chapter 5) and four different rat strains (chapter 6). Mast cells are present in the direct environment of blood vessels and can also be found disseminated throughout tissues.

The third part of the thesis focusses on the inhibition of leukocyte-endothelium interactions. Previous studies in our laboratory have shown that a thromboembolic reaction, i.e., the interaction between platelets and the vessel wall, decreases the level of leukocyte rolling downstream of the thrombus (oude Egbrink et al., 1992). In chapter 7 the influence of a thromboembolic reaction on leukocyte rolling is further elaborated with emphasis on the involvement of selectins, i.e., adhesion molecules necessary for the rolling of leukocytes along the endothelium. Since prostaglandins were suggested to be involved in the inhibition of leukocyte rolling (oude Egbrink et al., 1992), we investigated the effects of the prostaglandins $E_{1}, E_{2}, I_{2}$, and a cocktail of these three prostaglandins on leukocyte rolling and adhesion (chapter $\mathbf{8}$ ). In malignant tumors leukocyte-endothelium interactions are only infrequently found. Therefore, we investigated the effect of tumor derived angiogenic factors on leukocyte rolling and adhesion in the last experimental chapter (chapter 9). 
The concluding part of the thesis consists of a general discussion (chapter 10) in which the findings of the various chapters are discussed in an integral way, followed by a summary of the main conclusions. 



\section{LEUKOCYTE-ENDOTHELIUM INTERACTIONS; A LITERATURE SURVEY}

In the following chapter a literature survey will be presented about the development, differentiation and functions of leukocytes, about the functions of endothelial cells and about the interaction between leukocytes and the endothelium. Emphasis will be on the adhesion molecules involved in leukocyte-endothelium interactions and on the way these interactions can be modulated.

\subsection{Leukocytes}

When asking anyone about the function of white blood cells, the answer will have great resemblance to "they have something to do with defending our body against invading agents such as bacteria, viruses, and tumor cells." Cancer patients that are given the instruction to draw their leukocytes being at work in their body, make drawings of, for instance, little 'pacmans' eating their tumor, brushes cleaning a stain in a washbowl, or small police agents taking thiefs and burglers out of the body (Siegel, 1989). In fact, many kinds of white cells are morphologically and functionally distinguishable, cooperating with each other very cleverly and effectively in order to perform their various deeds or misdeeds (Van Arman, 1974). In the following paragraphs the various subtypes of leukocytes and their functions are summarized.

\subsubsection{Development and differentiation}

Leukocytes found in the blood can be morphologically divided into two groups: a) polymorphonuclear cells (PMNs), also called granulocytes, with as subtypes neutrophils, eosinophils and basophils, and b) monomorphonuclear cells (MMNs), with as subtypes lymphocytes and monocytes. Leukocytes as well as the red blood cells and blood platelets differentiate from pluripotent hematopoietic stem cells (see Figure 2.1).

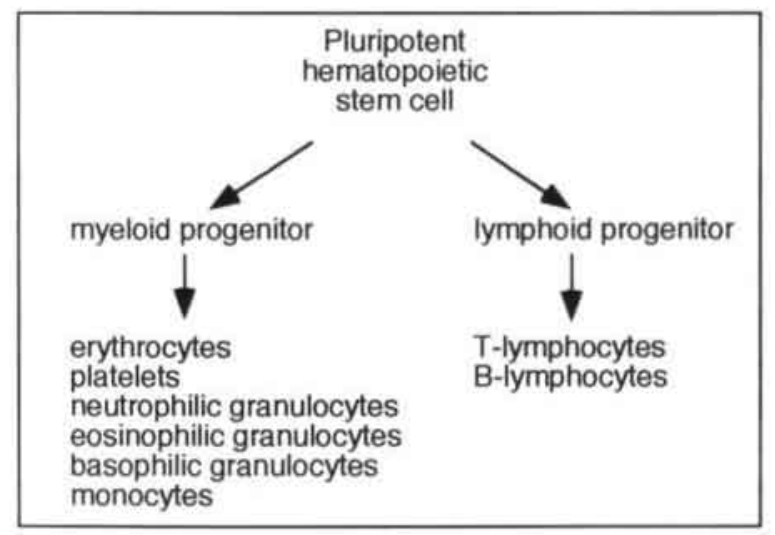

Figure 2.1. Differentiation of pluripotent stem cells into various leukocytes and other blood cells. 
These cells may give rise to restricted progenitor cells, among which those committed to the formation of myeloid cells and lymphoid cells. Myeloid stem cells give rise to red blood cells, platelets, PMNs as well as monocytes; lymphoid stem cells differentiate into various types of lymphocytes (see Figure 2.1). PMNs and monocytes are formed only in bone marrow, whereas lymphocytes are also produced in lymph glands, spleen, thymus and other lymphoid tissues (Haynes and Fauci, 1991; Guyton and Hall, 1996).

During maturation of PMNs specific cytoplasmic granules appear. Their neutrophilic, eosinophilic or basophilic characteristics allow histological identification of the three subtypes of granulocytes (Haynes and Fauci, 1991).

The total number of leukocytes per volume of blood as well as the percentages of the various subtypes vary per species. Table 2.1 shows the normal ranges found in humans and in the species used in this thesis. It is clear that a higher total number of leukocytes is found in the rat versus the other species and that in rodents a larger percentage of lymphocytes can be found as compared to humans. Within a few hours after the onset of acute inflammation, the number of leukocytes circulating in the blood will increase. In particular the relative proportion of neutrophils increases substantially, due to mobilization of stored neutrophils from the bone marrow into the circulation (Guyton and Hall, 1996).

Table 2.1. Ranges of total number of circulating leukocytes and percentages of leukocyte subtypes in several species.

\begin{tabular}{lllll}
\hline & Human & Rabbit & Rat & Mouse \\
total number $\left(\times 10^{6} / \mathrm{ml}\right.$ blood) & $3.5-11$ & $3-12$ & $6-18$ & $5-12$ \\
neutrophils (\%) & $40-70$ & $12-55$ & $14-20$ & $23-28$ \\
eosinophils (\%) & $0-5$ & $0-2.6$ & $1-4$ & $3-4$ \\
basophils (\%) & $0-1$ & $0-6.4$ & $0-0.5$ & 0 \\
monocytes (\%) & $1-10$ & $0-5.4$ & $1-6$ & $4-6$ \\
lymphocytes (\%) & $20-50$ & $40-85$ & $69-90$ & $61-67$ \\
\hline
\end{tabular}

References: Kozma et al., 1974; Ringler and Dabich, 1979; Rugh, 1991; van Zutphen et al., 1995; den Ottolander, 1997.

\subsubsection{Functions}

Each type of leukocyte described above is responsible for the mediation of a specific part of the host defence. In short, granulocytes and monocytes are involved in nonspecific destroying of invading agents by phagocytosis, the so-called innate immunity. Their reaction is not affected by prior contact with the infectious agent. Lymphocytes, on the other hand, are involved in the production of antibodies against specific invaders, the so-called acquired or adaptive immunity (Roitt, 1997).

\section{Polymorphonuclear leukocytes}

The most important function of neutrophilic granulocytes is phagocytosis of non-specific invaders. To this purpose, the cells have to leave the blood stream and move toward an inflamed area. There they can bind to receptors on the cells or particles to be 
destroyed and form pseudopodia in all directions around them. When the pseudopodia meet each other on the opposite side of the particle they fuse and create an enclosed vesicle containing the phagocytized particle. The vesicle eventually fuses with lysosomes, i.e., granules containing proteolytic enzymes, leading to digestion of the phagocytized particle. In addition, granulocytes can produce oxidizing agents such as superoxide $\left(\mathrm{O}_{2}{ }^{\circ}\right)$ and hydroxyl $\left(\mathrm{OH}^{\circ}\right)$ radicals, which are lethal to most bacteria. After having phagocytized 5 to 25 bacteria the granulocyte is killed by its own enzymes. The mixture of dead neutrophils and necrotic tissue is known as pus. Neutrophils usually circulate in the blood for 4 to 8 hours after recruitment from the bone marrow. They can live another 4 to 5 days in the tissue. During serious infections, however, their life span can be reduced to only a few hours (Guyton and Hall, 1996; Roitt, 1997).

Eosinophils are weak phagocytes; one of their main functions is to destroy various parasitic targets by releasing several enzymes. They are also found in large numbers in case of allergic reactions (Haynes and Fauci, 1991).

Basophils are able to release mediators like histamine, leukotrienes, platelet activating factor (PAF), and eosinophil chemotactic factor. Some of these mediators are released from granules, others are newly synthesized upon activation. Basophils and their related tissue mast cells are important in some types of allergic reactions, when they are stimulated to release their granule contents, causing local vascular and tissue reactions (Clark et al., 1977).

\section{Monomorphonuclear leukocytes}

Monocytes are immature cells with little capability to destroy invading agents. However, especially in cases of inflammation, they enter the tissue after a short time within the circulation, begin to swell and develop into macrophages, which are extremely defensive. In this form these cells can live for months or even years. Macrophages can phagocytize as many as 100 bacteria. The antigens of the bacteria are then presented to lymphocytes, activating those lymphocytes that recognize the antigen. Furthermore, macrophages secrete substances leading to the activation and proliferation of lymphocytes (Guyton and Hall, 1996; Roitt, 1997).

Lymphocytes can be divided into two major groups: T-lymphocytes and Blymphocytes. These subpopulations are thus classified, since T-lymphocytes mainly differentiate within the Thymus gland, whereas B-lymphocytes differentiate in the Bone marrow. Both are involved in the specific acquired immunity. T-lymphocytes can differentiate into various effector cells, among which T-helper and T-suppressor cells that are responsible for the regulation of the immune system. Cytotoxic T-cells, also called natural killer cells, directly attack cells that present specific foreign antigens; this is done through the release of cytotoxic substances. B-lymphocytes become activated through the presentation of antigens by macrophages. With the support of cytokines released by T-helper cells a B-lymphocyte can enlarge and proliferate into a plasma cell, which produces numerous antibodies against one specific agent (Roitt, 1997).

Lymphocytes are released into the circulation by drainage of lymph from lymph nodes. Lymphocytes are present in the blood stream for only a few hours. However, after they have migrated from the blood into tissues they will re-enter the blood stream 
through the lymph vessels. This process is called recirculation. Life spans of lymphocytes range from 100 days to years (Roitt, 1997).

\subsection{Endothelium}

The endothelium is the thin layer of single cells covering the luminal side of the wall of all blood vessels throughout the body. In an adult human being the endothelial cell surface is composed of approximately 1 to $6 \times 10^{13}$ cells, weighs approximately $1 \mathrm{~kg}$ and covers a vessel surface area of approximately 1 to $7 \mathrm{~m}^{2}$ (Cines et al., 1998). The various physiological functions of endothelial cells as well as their role in the pathophysiology of vascular disorders have been thoroughly reviewed (see for example Kirkpatrick et al., 1997; Cines et al., 1998). In short, endothelial cells are of utmost importance in the regulation of vascular permeability, vasoactivity (vasodilation as well as vasoconstriction), angiogenesis, coagulation (antithrombotic as well as prothrombotic properties have been described), and the interaction with blood cells (Kirkpatrick et al., 1997; Cines et al., 1998). One should realize, however, that the endothelium comprises a heterogeneous population of cells (Gerritsen, 1987; Bicknell, 1993; Cines et al., 1998). Morphological and/or functional differences have been described between macrovascular and microvascular endothelium, between arterial and venous endothelium, between arteriolar and venular endothelium, and between endothelium in different vascular beds. Therefore, data obtained from only one type of endothelium should not be generalized.

\subsection{Leukocyte-endothelium interactions}

For granulocytes to exert their phagocytic function in inflamed tissue, for monocytes to become tissue macrophages, and for lymphocytes to recirculate, these cells have to move via the blood to the area in the body where they are needed and, subsequently, they have to leave the circulation. The process of leukocyte-endothelium interactions that underlies this migration appears to be a multistep cascade: each step has to be completed for the next step to occur (Butcher, 1991; von Andrian and Arfors, 1993; Springer, 1994). The general process with its various steps is illustrated in Figure 2.2. First, substances released at the site of inflammation activate the local endothelial cells to express adhesion molecules. The release is rather instantaneous, but the expression may take some time. In case leukocytes marginate from the (center of the) blood stream in the direction of the vessel wall, they weakly bind to the activated endothelium and start to roll along the vessel wall. Due to their longer transit time in the inflamed area, the leukocytes have the time to become activated if necessary, which marks the next step: they stop rolling and firmly adhere to the endothelium. Thereupon they may change shape, and migrate through the vessel wall into the inflamed tissue. This cascade of leukocyte-endothelium interactions normally occurs in the microcirculation, and more specifically in the postcapillary venules.

In the following paragraphs the various steps and the adhesion molecules involved in the processes from leukocyte margination to diapedesis will be described more 


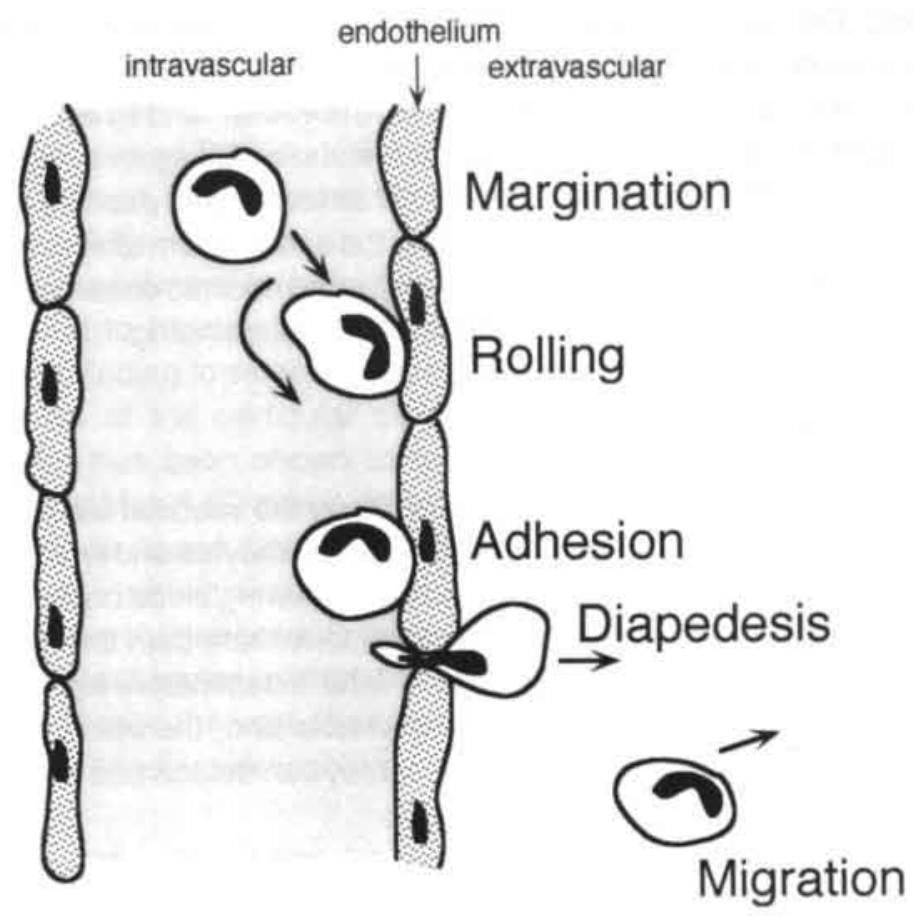

Figure 2.2. The various steps leading to leukocyte infiltration into inflamed tissue. Leukocytes marginating from the blood stream toward the vessel wall may weakly bind to activated endothelium and start rolling along the vessel wall. When the leukocytes become activated they can firmly adhere to the endothelium, change shape, and migrate through the vessel wall (diapedesis) into the surrounding tissue.

extensively. Focus will be on the adhesion molecules involved in the interaction between granulocytes and endothelium, because in the mesentery, i.e., the tissue most frequently used in this thesis, $98-100 \%$ of the leukocytes rolling in postcapillary venules are granulocytes (Tangelder et al., 1995).

\subsubsection{Leukocyte margination}

Margination of leukocytes in venules is supposed to be based on a hydrodynamic interaction between red and white cells (Schmid-Schönbein et al., 1980). In capillaries leukocytes tend to flow at a slower rate than erythrocytes, among others due to their larger mass. Erythrocytes accumulate behind a leukocyte, leaving a cell depleted region in front of it. When this leukocyte then enters a wider venule, the red blood cells will pass and drive the slower leukocyte away from the central blood stream toward the wall. These collisions may bring leukocytes in close proximity to the vascular lining (SchmidSchönbein et al., 1980). Another mechanism could be that leukocytes that inevitably interact with the endothelium of the small capillaries, stay in touch with the endothelium from the moment they enter the larger postcapillary venules due to adhesion molecule 
binding. On the other hand, various forces tend to displace the leukocytes back to the central blood stream: a) larger particles flowing in the blood tend to stream where the shear forces are low, i.e., in the center of the vessel, and b) leukocytes and endothelial cells both have negatively charged surfaces, resulting in a repulsive force between them (von Andrian and Arfors, 1993). Of all leukocytes passing a postcapillary venule a substantial number actually rolls along the endothelium after endothelial activation by, for example, exteriorization of the tissue for microscopic observation (Ley et al., 1991a). This indicates the strength of adhesion molecule binding.

\subsubsection{Leukocyte rolling}

The rotational movement of leukocytes along the vascular wall that is called rolling, is the result of a weak adhesive force between leukocytes and endothelial cells on the one hand and the propulsive force exerted by the flowing blood on the other. Leukocytes roll on vascular endothelium at a significantly lower rate than the free flowing blood cells. It seems that they explore the vessel wall for inflammatory signs. The weak binding of leukocytes with the endothelium is reversible and, therefore, cells can be observed rolling as well as jumping or saltating, or they can detach and return into the main blood stream.

Leukocyte rolling is mediated by adhesion molecules of the selectin family (extensively reviewed in McEver, 1991; Lasky, 1992; Crockett-Torabi, 1998; Vestweber and Blanks, 1999). Up till now, three selectins are known: L-selectin on Leukocytes, Eselectin on Endothelial cells, and P-selectin on Platelets and endothelial cells. The structure of these three selectins is roughly similar (Figure 2.3): they contain an $\mathrm{N}$ -

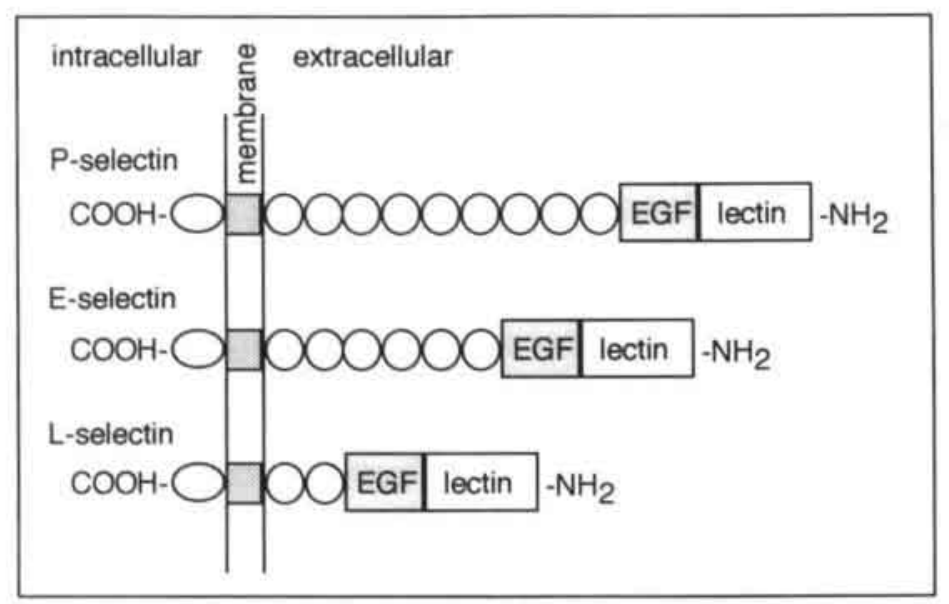

Figure 2.3. Structure of the three human selectins. All selectins have a short carboxyl terminal cytoplasmic sequence, a transmembrane domain, a variable number of complement regulatory-like modules, an epidermal growth factor (EGF)-like domain, and an amino terminal domain which is homologous to C-type lectins. 
terminal $\mathrm{Ca}^{2+}$-dependent lectin domain, an epidermal growth factor (EGF)-like domain, a series of complement-like repeat domains (in humans: 2 repeats in L-selectin, 6 in Eselectin, and 9 in P-selectin), and a transmembrane region followed by a short cytoplasmic tail (Vestweber and Blanks, 1999). Cell-cell interactions are mediated by the N-terminal lectin domain in a calcium dependent manner (Geng et al., 1991; Anostario and Huang, 1995).

In addition to their involvement in leukocyte rolling, recently selectins and their ligands have been found to function as signaling receptors as well (see for review Crockett-Torabi, 1998). Binding to selectins can initiate intracellular signals leading to enhanced adhesiveness of the particular cell involved. For instance, binding of antibodies to L-selectin has been shown to upregulate in leukocytes the surface expression of the integrin Mac-1 (Crockett-Torabi et al., 1995); this also holds for the binding of L-selectin to its ligand GlyCAM-1. The biochemical signal transduction pathways that play a role in selectin-mediated leukocyte activation have not yet been elucidated, although recent studies have demonstrated that an increase in intracellular calcium concentration in leukocytes is involved (Crockett-Torabi, 1998).

In the following paragraphs the way of expression of the three selectins, and the ligands to which they can bind are described.

\section{L-selectin}

L-selectin (CD62L), also formerly known as lymphocyte homing receptor, leukocyte adhesion molecule-1 (LAM-1), or lectin-like cell adhesion molecule-1 (LECAM-1 or LECCAM-1), is constitutively expressed on the cell surface of myeloid cells and a large subset of lymphocytes (Lewinsohn et al., 1987). It was first reported to mediate the binding of lymphocytes to high endothelial venules (HEV) of peripheral lymph nodes (Gallatin et al., 1983), but soon it was shown to mediate neutrophil-endothelium interactions in vitro (Spertini et al., 1991) and in vivo (Ley et al., 1991b; von Andrian et al., 1991) as well. L-selectin appears to mediate leukocyte rolling independent of $E$-and P-selectin (Ley et al., 1993), but it also presents oligosaccharide ligands to E- and Pselectin (Picker et al., 1991). This has led to the concept that L-selectin acts as an adhesion molecule in two ways: by recognizing endothelial ligands and at the same time by presenting ligands to endothelial selectins (von Andrian et al., 1993b).

On human neutrophils, on average $78 \%$ of $\mathrm{L}$-selectin is clustered on the microvilli (Bruehl et al., 1996). This topography is necessary for the ability of a cell to interact with other cells under flow conditions (von Andrian et al., 1995).

Upon activation of leukocytes L-selectin is shed from the cell surface by proteolytic cleavage within 4 minutes (Kishimoto et al., 1989). At the same time adhesion molecules involved in firm adhesion of leukocytes are upregulated, promoting adhesion and subsequent migration into tissues (Kishimoto et al., 1989; McEver, 1991; Neeley et al., 1993). It has been shown that shedding of L-selectin is not required for leukocyte migration across endothelial monolayers (Allport et al., 1997).

\section{P-selectin}

P-selectin (CD62P), also known as granule membrane protein of molecular weight 140 
kD (GMP140), or platelet activation-dependent granule external membrane protein (PADGEM), is stored in alpha-granules in platelets (Stenberg et al., 1985) and in Weibel-Palade bodies in endothelial cells (Hattori et al., 1989). Upon activation these storage granules fuse with the plasma membrane, thus redistributing P-selectin to the cell surface (Stenberg et al., 1985). This can occur very rapidly: in vitro, maximal expression was found at 3 minutes after stimulation. Expression declines to baseline levels in 20 minutes (Hattori et al., 1989). The reduction is most likely the result of endocytosis. Due to this so-called internalization P-selectin returns to the storage granules from where it can be reused (Subramaniam et al., 1993).

Several endogenous and exogenous mediators have been shown to stimulate endothelial cells to express P-selectin, among which are histamine (Hattori et al., 1989; Jones et al., 1993), thrombin (Hattori et al., 1989; Zimmerman et al., 1994), plasmin (Montrucchio et al., 1996), oxidized low-density lipoprotein (ox-LDL) (Gebuhrer et al., 1995), oxygen radicals (Patel et al., 1991), phorbol esters (Hattori et al., 1989), calcium ionophores (Hattori et al., 1989), and leukotriene $\mathrm{C}_{4}$ and $\mathrm{D}_{4}$ (Datta et al., 1995). Some mediators have been shown to regulate P-selectin expression at the transcriptional level. For instance, nitric oxide (NO) has been suggested to diminish endothelial Pselectin expression, since NO-synthase inhibitors increase P-selectin mRNA with a peak effect after 2 to $4 \mathrm{~h}$ (Davenpeck et al., 1994; Armstead et al., 1997). Evenso, the cytokine IL-3, produced by T-lymphocytes, has been shown to stimulate long-term expression of $\mathrm{P}$-selectin that is accompanied by an increase in P-selectin mRNA (KhewGoodall et al., 1996).

The role of P-selectin in leukocyte rolling has been demonstrated first in vitro on activated human endothelial monolayers (Geng et al., 1990; Jones et al., 1993) and later in vivo in the dog mesentery (Doré et al., 1993). Important support for its role was given by the observation that leukocyte rolling was severely reduced in mesenteric venules of P-selectin deficient mice (Mayadas et al., 1993). In these same mice, recruitment of neutrophils to the peritoneal cavity upon inflammation was delayed, demonstrating the importance of rolling as a prerequisite for subsequent extravasation (Mayadas et al., 1993). The role of P-selectin has been shown to be greatest in initial leukocyte rolling, while at later time points L-selectin is involved more prominently (Ley et al., 1995).

\section{E-selectin}

E-selectin (CD62E) was identified as inducible endothelial-leukocyte adhesion molecule-1 (ELAM-1) (Bevilacqua et al., 1987). In contrast to P-selectin, E-selectin is not stored in granules, but requires de novo mRNA and protein synthesis to be expressed on the cell surface. Expression of E-selectin requires stimulation of the endothelial cells with such cytokines as interleukin-1 (IL-1), tumor necrosis factor-alpha (TNF $\alpha$ ), and lipopolysaccharide (LPS) (Bevilacqua et al., 1987). In vitro, the expression reaches its maximum between 2 and 6 hours and it declines within 24 hours (Bevilacqua et al., 1989). The decline is due to the release of E-selectin, as indicated by the presence of soluble E-selectin upon activation of the endothelial cells (Leeuwenberg et al., 1992), or to internalization after which E-selectin is degraded in lysosomes 
(Subramaniam et al., 1993). E-selectin expression can also be upregulated in vivo by for example interleukin-1 (IL-1) (Olofsson et al., 1994). In some tissues it appears to be constitutively present (Keelan et al., 1994; Janssen, 1999).

Involvement of E-selectin in leukocyte rolling has been established in vitro under flow conditions (Abbassi et al., 1993; Lawrence and Springer, 1993), as well as in vivo in IL-1 stimulated rabbit mesenteric venules (Olofsson et al., 1994). Rolling on E-selectin appears to be slower than rolling on P-selectin, indicating a stronger interaction between leukocytes and endothelium in case of involvement of the former selectin. The slower rolling results in longer leukocyte transit times, a prerequisite for efficient leukocyte adhesion (Kunkel and Ley, 1996; Ley et al., 1998).

\section{Selectin ligands}

Selectins bind to ligands, i.e., counterreceptors (Table 2.2), on opposing cells which contain sialylated oligosaccharides; these oligosaccharides are recognized by the lectin-like domains of the selectins (McEver, 1991). The first ligands discovered were sialyl Lewis X $\left(s^{x} e^{X}\right)$ (Phillips et al., 1990) and its related sialyl Lewis a $\left(s L e^{a}\right)$. Both sialic acid and $\mathrm{Le}^{\mathrm{x}}$ are components of all selectin ligands. For each selectin high affinity ligands have been identified with distinct structures, but overlapping specificities (Larsen et al., 1992; Crockett-Torabi, 1998). Binding of oligosaccharides to selectins appears to be $\mathrm{Ca}^{2+}$ - and pH-dependent (Anostario et al., 1994).

Table 2.2. Adhesion molecules involved in leukocyte-endothelium interactions: selectins and their counterreceptors

\begin{tabular}{|c|c|c|}
\hline Selectin & Distribution & Counterreceptors \\
\hline L-selectin & $\begin{array}{l}\text { granulocytes, monocytes, } \\
\text { subset of lymphocytes }\end{array}$ & $\begin{array}{l}\text { GlyCAM-1, CD34, MadCAM-1, Sgp } 200 \\
\text { P-selectin, E-selectin }\end{array}$ \\
\hline P-selectin & $\begin{array}{l}\text { activated endothelial cells, } \\
\text { activated platelets }\end{array}$ & L-selectin, PSGL-1, CD24 \\
\hline E-selectin & activated endothelial cells & $\begin{array}{l}\text { L-selectin, PSGL-1, ESL-1, CD66, } \\
\beta_{2} \text {-integrins }\end{array}$ \\
\hline
\end{tabular}

As mentioned above, L-selectin can bind to the two endothelial selectins, $\mathrm{P}$ - and $\mathrm{E}$ selectin. In addition, four specific ligands for $L$-selectin have been described. Two of them were first discovered as the sulfated glycoproteins Sgp ${ }^{50}$ and Sgp ${ }^{90}$ (Rosen, 1993), and are now designated glycosylation-dependent cell adhesion molecule-1 (GlyCAM-1) and CD34, respectively (Rosen, 1993; Crockett-Torabi, 1998). GlyCAM-1 is constitutively expressed on the endothelium of high endothelial venules (HEV) in peripheral lymph nodes, but can also be released into the circulation where it may inhibit L-selectin mediated leukocyte rolling (Tedder et al., 1995). CD34 is present on most endothelial cells as well as on hematopoietic stem cells (Young et al., 1995). A third L-selectin ligand is the mucosal addressin cell adhesion molecule-1 (MadCAM-1). which also serves as a ligand for specific integrin molecules (Berg et al., 1993); this adhesion molecule is expressed on HEV in mucosal lymph nodes. Recently, a fourth Lselectin binding sulfated glycoprotein, designated as $\mathrm{Sgp}^{200}$, has been discovered, but this molecule has not been cloned yet (Kawashima et al., 1998; Vestweber and Blanks, 
1999). Potential ligands for L-selectin have been demonstrated on other than endothelial cells as well. An example are the myelin sheaths in the central nervous system. This may explain the targeting of leukocytes to certain tissues in immunopathological reactions such as multiple sclerosis (Rosen, 1993; Kawashima et al., 1998).

P-selectin binds to oligosaccharides, as presented by L-selectin (Picker et al., 1991), and to a high affinity ligand, called P-selectin glycoprotein ligand-1 (PSGL-1 or CD162) (Crockett-Torabi, 1998; Vestweber and Blanks, 1999). PSGL-1 is constitutively expressed by neutrophils (where it is localized on the microvilli; Moore et al., 1995), lymphocytes and monocytes. However, a structural modification is required for ligand function (see for review Moore, 1998). The importance of PSGL-1 in vivo has been demonstrated by the fact that administration of an antibody against PSGL-1 reduces the rolling of human PMNs in rat mesenteric venules (Norman et al., 1995). PSGL-1 also functions as a ligand for E-selectin. Recently, CD24 on myeloid cells has also been described as a ligand for P-selectin (Vestweber and Blanks, 1999).

Beside its affinity for L-selectin and PSGL-1, E-selectin binds to a specific ligand on myeloid cells, termed E-selectin ligand-1 (ESL-1), which has a large homology with a receptor for fibroblast growth factor (Steegmaier et al., 1995). Other glycoproteins on leukocytes, that bind to E-selectin, have been identified, but as yet not cloned (Vestweber and Blanks, 1999). E-selectin has also been shown to bind to CD66 (Kuijpers et al., 1992b) and to a subpopulation of $\beta 2$-integrins which carry sLe ${ }^{x}$ (Kotovuori et al., 1993).

\subsubsection{Leukocyte activation}

The initial rolling of leukocytes is transient, unless the leukocytes are activated by chemoattractants or cell contact-mediated signals capable of triggering secondary adhesion molecules (Butcher, 1991). Substances that can activate leukocytes are, among others, phorbol myristate acetate (PMA), complement factor C5a, TNF $\alpha$, leukotriene $\mathrm{B}_{4}\left(\mathrm{LTB}_{4}\right)$, LPS, IL-1, and PAF (Kishimoto et al., 1989). A study of Lorant and coworkers shows that P-selectin and PAF, which are coexpressed by activated endothelial cells, work together: P-selectin captures a leukocyte, thus facilitating interaction between receptors on the leukocyte and the signaling molecule PAF. This leads to an elevation of intracellular $\mathrm{Ca}^{2+}$ and activation of the leukocyte (Lorant et al. 1993). Subsequently, the leukocytes change shape within seconds and become flattened (Adams and Shaw, 1994). In this way they interact with a larger surface of the vessel wall. Upon activation the affinity and the expression of adhesion molecules of the integrin family on the leukocytes are greatly increased (Kishimoto et al., 1989; see also paragraph 2.3.4). In contrast, L-selectin is shed from the cell surface upon activation. This explains why leukocytes derived from, for example, bronchoalveolar lavage or inflamed tissue, i.e., leukocytes that have migrated through the vessel wall and therefore must have been activated, show only little L-selectin expression (Kishimoto et al., 1989; Mengelers et al., 1993). 


\subsubsection{Leukocyte adhesion}

Firm adhesion of leukocytes to the endothelium of post-capillary venules is mediated by adhesion molecules of two families: the integrins, and the immunoglobulin gene superfamily (see Table 2.3).

\section{Integrins}

Integrins are composed of an $\alpha$-and a $\beta$-subunit, which are non-covalently linked. The integrins with the $\beta_{2}$ subunit (CD18) are solely expressed on leukocytes. At present, four $\beta_{2}$ integrins have been described (for reviews see (Albelda and Buck, 1990; Arnaout, 1990; Gahmberg et al., 1997; Gahmberg et al., 1998): CD11a/CD18 (also known as lymphocyte function-related antigen-1, LFA-1, or $\alpha_{L} \beta_{2}$ ), CD11b/CD18 (membrane attack complex-1, Mac-1, or $\left.\alpha_{M} \beta_{2}\right), \operatorname{CD} 11 \mathrm{c} / \mathrm{CD} 18$ (p150,95 or $\left.\alpha_{x} \beta_{2}\right)$, and CD11d/CD18 $\left(\alpha_{D} \beta_{2}\right)$. These integrins are present on the cell surface of several types of leukocytes, but also in intracellular granules, from which they can be translocated to the cell membrane upon stimulation (Gahmberg et al., 1997). The integrins are not constitutively active, but can be upregulated through an increase in the number of molecules expressed on the cell surface. Their activity can also be enhanced through conformational changes increasing their binding affinity. Under physiological circumstances the trigger for this comes from outside the cell, through binding of an agonist to the integrins or to other receptors on the cell surface, followed by intracellular signal transduction (Crockett-Torabi et al., 1995; Gahmberg et al., 1997; Crockett-Torabi, 1998; Gahmberg et al., 1998).

Other integrins, such as very late activation antigen-4 (VLA-4; $\alpha_{4} \beta_{1}$ ) and lymphocyte Peyer's patch adhesion molecule-1 (LPAM-1; $\alpha_{4} \beta_{7}$ ), are involved in the homing of lymphocytes, but are not expressed on neutrophils (Springer, 1994).

Table 2.3. Adhesion molecules involved in leukocyte adhesion and diapedesis with their counterreceptors: the integrin family and immunoglobulin gene superfamily.

\begin{tabular}{lll}
\hline Name & Distribution & Counterreceptors \\
\hline Integrin family: & all leukocytes & ICAM-1, ICAM-2, ICAM-3 \\
LFA-1 & granulocytes, monocytes & ICAM-1, ICAM-2, ICAM-3 \\
Mac-1 & granulocytes, monocytes & ICAM-1 \\
p150,95 & monocytes, lymphocytes, eosinophils & VCAM-1, fibronectin \\
VLA-4 & subset of lymphocytes & MadCAM-1, VCAM-1, \\
LPAM-1 & & \\
fibronectin & & \\
& & \\
Immunoglobulin gen & superfamily: & \\
ICAM-1 & activated endothelial cells and leukocytes & LFA-1, Mac-1, P150,95 \\
ICAM-2 & endothelial cells, leukocytes & LFA-1, Mac-1 \\
ICAM-3 & resting leukocytes & LFA-1, Mac-1 \\
VCAM-1 & activated endothelial cells, macrophages & VLA-4, LPAM-1 \\
MadCAM-1 & endothelial cells of mucosal HEV & L-selectin, LPAM-1 \\
PECAM-1 & endothelial cells, leukocytes, platelets & PECAM-1 \\
\hline
\end{tabular}




\section{Immunoglobulin gene superfamily}

Counterreceptors for the integrins are members of the immunoglobulin gene superfamily, a family of glycoproteins with multiple immunoglobulin-like domains (the structure and function of this family of adhesion molecules is reviewed in Huang et al., 1997). For leukocyte adhesion to the endothelium the following members of this group are of importance (Gahmberg et al., 1997): the intercellular cell adhesion molecules (ICAM-1, ICAM-2, and ICAM-3), vascular cell adhesion molecule-1 (VCAM-1), MadCAM-1 (one of the ligands for L-selectin, see paragraph 2.3.2.), and plateletendothelial cell adhesion molecule-1 (PECAM-1). ICAM-1 is present at low levels on unstimulated endothelial cells, but VCAM-1 is not. The expression of these molecules is rapidly induced after activation of the endothelial cells by cytokines (Imhof and Dunon, 1997). ICAM-2 is constitutively expressed on the endothelium at high levels and is not upregulated through activation (Imhof and Dunon, 1997). ICAM-3 is absent on normal endothelial cells, but highly present on, for example, lymphoma cells (Gahmberg et al., 1997). The ICAMs serve as counterreceptors for the $\beta_{2}$-integrins; VCAM- 1 for the $\alpha_{4}$-integrins. Apart from binding to $\mathrm{L}$-selectin, MadCAM-1 also functions as a counterreceptor for the integrin $\alpha_{4} \beta_{7}$ (Vestweber and Blanks, 1999). PECAM-1 is constitutively expressed on endothelial cells, leukocytes and platelets and is among others involved in diapedesis of leukocytes (Imhof and Dunon, 1997; see paragraph 2.3.5).

\subsubsection{Diapedesis and migration}

\section{Diapedesis}

Chemoattractants are required for transendothelial migration of leukocytes (Springer, 1994). Several chemoattractants have been studied in in vitro assays as well as in vivo (see for example: Kuijpers et al., 1992a; Bienvenu et al., 1994; Nourshargh et al., 1995). Members of both the integrin and the immunoglobulin family involved in leukocyte adhesion are also involved in leukocyte transmigration (Collins, 1995). An example is PECAM-1, which is present at the intercellular junctions between endothelial cells and is also expressed by leukocytes. One of the characteristics of PECAM-1 is that homophilic interaction can take place: PECAM-1 on the leukocytes can bind to PECAM1 on the endothelial cells (Collins, 1995), thereby facilitating leukocyte transmigration.

\section{Migration}

Once migrated from the blood stream into the tissue, leukocytes have to migrate through the tissue in the direction of the offending agents, such as bacteria or (injected) chemoattractants. It has been proposed that in their movement through the interstitial matrix proteolysis plays a role: enzymes such as elastase that are released by the leukocytes degrade matrix components, thereby facilitating passage through the tissue (Bienvenu et al., 1994). The oriented locomotion, i.e., chemotaxis, is due to sensing of a chemical gradient. The leukocyte senses the concentration of a chemotactic substance at two or more locations on its cell surface at the same time and moves in the direction of the highest concentration (Zigmond, 1974). After arrival at this site the cells can start to exert their function of phagocytosis. 


\subsubsection{Sequential contribution of adhesion molecules}

Figure 2.4 presents a summarizing, schematic view of the most likely sequential contribution of adhesion molecules to leukocyte-endothelium interactions. In short, after activation of endothelial cells, they release P-selectin (within minutes) and express Eselectin (within 2 to 4 hours), and leukocytes can start rolling. Rolling can also be supported by adhesion molecules other than selectins, such as ICAM-1 (Kunkel et al.. 1996; Steeber et al., 1998). The close proximity of the rolling leukocytes to the endothelium facilitates their activation by endothelial cytokines, which is followed by an increase in the number of integrins and a conformational change of these adhesion molecules. Subsequently, the integrins bind tightly to adhesion molecules of the immunoglobulin gene superfamily, which are abundantly present on activated endothelial cells. This is followed by transmigration of leukocytes through intercellular junctions between endothelial cells.

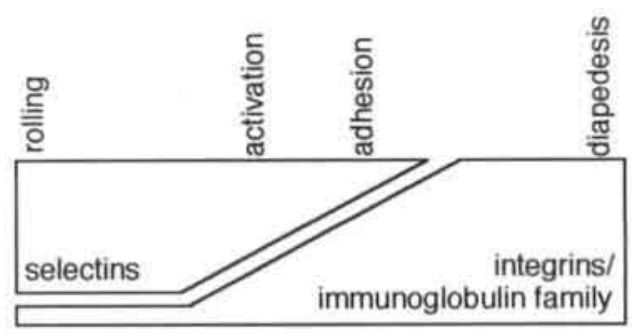

Figure 2.4. Schematic representation of the sequential contribution of selectins, integrins, and adhesion molecules of the immunoglobulin gene superfamily in the various steps leading to rolling, adhesion, activation and diapedesis of leukocytes.

\subsubsection{In vitro versus in vivo}

A considerable number of studies on leukocyte-endothelium interactions has been performed in in vitro models using cultured endothelial cells of various origin and isolated leukocytes of various subtypes. Beside the possibility to control experimental conditions, one of the advantages of in vitro systems is that it allows the use of human tissues or cells. Most in vitro studies use endothelial cells derived from large vessels, such as human umbilical vein endothelial cells (HUVECs). It has been shown, however, that endothelial cells derived from small vessels are phenotypically different from endothelial cells derived from large vessels (Swerlick and Lawley, 1993). Therefore, techniques have been developed for isolation and culture of microvascular endothelial cells. However, a high level of heterogeneity exists among endothelial cells from various vessels (as described in paragraph 2.2). This appears to be mainly due to signals from the environment (Gerritsen, 1987). Functions and activities are modified according to the quantity and/or quality of these signals (Pober and Cotran, 1990). In in vitro systems signals from the environment are rather different as compared to the in vivo situation. Therefore, cells will behave differently under these circumstances. In addition, culturing 
of endothelial cells over several passages will affect their phenotype; long term culturing may induce dedifferentiation. This is illustrated by the fact that on human umbilical artery endothelial cells of early passage the expression of E-selectin, VCAM-1 and ICAM-1 can be induced by cytokines, while on cells of late passage expression of these adhesion molecules could not be induced by cytokines (Bartlett and Parish, 1995).

One of the advantages of using isolated leukocytes in in vitro models is that studies can be performed with several subtypes of leukocytes. However, the procedures used to isolate the cells may induce functional changes themselves. To study the effect of isolation procedures, neutrophil function and adhesion molecule expression was determined in whole blood and in cell suspensions isolated by two different procedures (Watson et al., 1992). Neutrophils isolated by either method showed, among others, increased expression of CD11b compared with those in whole blood, suggesting that the cells were activated by the isolation procedures, possibly by processes analogous to those induced by cytokines (Watson et al., 1992). In vivo research allows us to study the behavior of leukocytes in their natural environment and in their relatively 'normal' activation state.

The possibilities to culture endothelial cells and to isolate leukocytes have led to the development of leukocyte adhesion assays in vitro. These adhesion assays can be performed under static conditions or under flow conditions mimicking the hemodynamic conditions in vivo. In many of these studies, however, leukocytes are perfused through flow chambers with an endothelial cell monolayer at wall shear stresses of about 2 dynes $/ \mathrm{cm}^{2}$ (see for example: Abbassi et al., 1993; Jones et al., 1994), since a higher wall shear stress (of about 4 dynes $/ \mathrm{cm}^{2}$ ) reduces leukocyte-endothelium interactions to virtually zero (Lawrence et al., 1987). In vivo, however, wall shear stresses are estimated to be about $18 \mathrm{dyn} / \mathrm{cm}^{2}$ in arterioles (Reneman et al., 1992) and $7.2 \mathrm{dyn} / \mathrm{cm}^{2}$ in venules (Reneman, unpublished observations) of 15-40 $\mu \mathrm{m}$ diameter. In these venules leukocyte-endothelium interactions do occur. This indicates that the balance between adhesive and propulsive forces is different in the in vitro situation as compared to in vivo situation; in vitro adhesion forces appear to be weaker.

Studies concerning the movement of leukocytes toward a chemotactic gradient are mainly based on in vitro models. In some studies two-dimensional systems were used, but models to study three-dimensional movements have been developed as well (Bienvenu et al., 1994). Endothelial cell monolayers are grown confluently on a filter and leukocytes applied to the 'luminal' side of the layer are observed migrating into the lower component of the chamber, which contains a chemotactic cytokine (Smith, 1993). Although in this manner the movement of leukocytes through a layer of endothelial cells and through physiologically relevant matrices can be studied, these kind of systems do not provide direct assessment of leukocyte kinetics within a true interstitial matrix in vivo (Bienvenu et al., 1994).

Menger and Lehr state that "the petri dish can never adequately model the full dimensions of integrated local and systemic positive and negative feedback loops in the control and regulation of complex physiological or pathophysiological processes" (Menger and Lehr, 1993). The development of intravital microscopic techniques has provided us with means to study leukocyte-endothelium interactions in vivo, where 
leukocytes as well as endothelial cells are in their natural environment and where their interaction can be influenced by environmental factors. In the present thesis these techniques have been used and leukocyte-endothelium interactions were studied in vivo.

\subsubsection{Venules versus arterioles}

Leukocyte rolling and adhesion is normally observed in venules, and almost never in arterioles. Different hemodynamic conditions in venules and arterioles could account for the absence of leukocyte-endothelium interactions in arterioles under normal circumstances. From our observations (Tangelder et al., 1993) and those of others (Ley and Gaehtgens, 1991), however, it may be concluded that in the mesentery this marked difference between venules and arterioles persists even at similar RBC velocities and shear rates. Therefore, hemodynamic differences between arterioles and venules can be ruled out as a major explanation for the absence of leukocyte-endothelium interactions in arterioles. Instead, it has been proposed that a distinct distribution of endothelial adhesion molecules may account for the preferential interaction of leukocytes with venular endothelium (Ley and Gaehtgens, 1991). Indeed, in vitro as well as ex vivo it has been shown that venular and arteriolar endothelial cells differ in their expression of adhesion molecules (Bartlett and Parish, 1995; Jung and Ley, 1997). For example, histological evaluation of the mouse cremaster muscle reveales that P-selectin is present on venular endothelium within 10 minutes after exteriorization and ICAM-1 is constitutively expressed, whereas none of the two adhesion molecules is expressed on the arteriolar endothelium in this tissue. E-selectin is expressed to a limited extent on venules but not on arterioles in mouse cremaster muscle. After cytokine treatment all three adhesion molecules are upregulated on venular endothelial cells. By contrast, after treatment of tissue with TNF $\alpha$, arterioles only express P-selectin, but not Eselectin or ICAM-1 (Jung and Ley, 1997). The latter observation is in accordance with observations in vivo in the same tissue: P-selectin dependent leukocyte rolling in arterioles is only observed following exposure of the tissue to TNF $\alpha$ (Thorlacius et al., 1997).

\subsubsection{Leukocyte adhesion deficiency}

The functional importance of interactions between leukocytes and the vessel wall is demonstrated in patients with a disease called leukocyte adhesion deficiency (LAD). Untill now several subtypes of LAD have been described. LAD-1 is an autosomalrecessive trait characterized by a congenital defect in the adhesion molecules LFA-1 and Mac-1: the subunit CD18 that is shared by these two integrin molecules on leukocytes is deficient (Anderson and Springer, 1987). A mild variant with normal expression of CD11/CD18 but impaired activation of this adhesion molecule has also been described (Kuijpers et al., 1997). In LAD-2 sialyl Lewis $X\left(s L e^{X}\right)$, the ligand for selectins, appears to be absent due to a glycosylation defect (Etzioni et al., 1992). The clinical symptoms of patients with LAD-1 or LAD-2 are similar: patients suffer from 
recurrent episodes of severe bacterial infections. Characteristically, these infections lack the formation of pus despite markedly elevated systemic leukocyte counts (Etzioni et al., 1992; von Andrian et al., 1993a). Patients with severe deficiencies have a high chance to die in infancy, and those surviving the early years demonstrate a susceptibility to severe, life-threatening systemic infections (Anderson and Springer, 1987). The in vivo behavior of neutrophils from LAD-1 and LAD-2 patients has been studied by von Andrian and coworkers (von Andrian et al., 1993a). The CD18-deficient leukocytes appeared to roll normally, but failed to adhere to the vessel wall upon activation, or to migrate in the surrounding tissues when challenged with a chemotactic stimulus. The leukocytes without $s L e^{x}$ were severely affected in both their ability to roll and to adhere to the vessel wall. In conclusion, leukocyte adhesion deficiency syndromes provide tools to study the impaired interactive functions of leukocytes and demonstrate the clinical importance of the ability of leukocytes to roll along and adhere to the vascular endothelium.

\subsection{Modulation of leukocyte-endothelium interactions}

In the following paragraphs cells, substances and/or processes that have been described to modulate (induce or inhibit) leukocyte-endothelium interactions and that are studied in this thesis are presented.

\section{Mast cells}

Mast cells (reviewed in Raud, 1989; Bernstein and Lawrence, 1990; Galli, 1993) were first identified in the frog mesentery by von Recklinghausen in 1863, but they received their name from Ehrlich (1878), who thought they were well-fed cells and assigned them a nutritional role. In fact, mast cells more closely resemble land mines (Levy, 1996). The cells are present throughout the body, especially in loose connective tissue and skin, and can be found predominantly in the neighbourhood of blood vessels. Their granules contain such mediators as histamine and enzymes, that can be released upon activation of the cells. Biologically active factors newly synthesized and released by mast cells include such lipid mediators as prostaglandins, leukotrienes and PAF, and such cytokines as TNF $\alpha$ (Galli, 1993).

Figure 2.5 illustrates the way in which mast cells may contribute to the sequential steps in leukocyte-endothelium interactions. Firstly, leukocyte rolling is supposed to be induced by the release of histamine from activated, degranulated mast cells which may cause redistribution of P-selectin to the endothelial cell surface (see paragraph 2.3.2) (Hattori, et al., 1989). Regarding the histamine receptor that is involved in the induction of leukocyte rolling in vivo, contradictory data have been published. Some studies state that the effect is $\mathrm{H}_{1}$-receptor mediated (Asako et al., 1994; Kubes and Kanwar, 1994; Gaboury et al., 1995), while in another study the $\mathrm{H}_{2}$-receptor was thought to be involved (Ley, 1994). Leukocyte rolling may also be induced by expression of E-selectin on endothelial cells upon stimulation by mast cell derived TNF $\alpha$ (Bevilacqua et al., 1987). Indeed, shortly after surgical preparation of the mouse cremaster muscle, E-selectin can only be immunohistochemically demonstrated in the direct neighbourhood of 


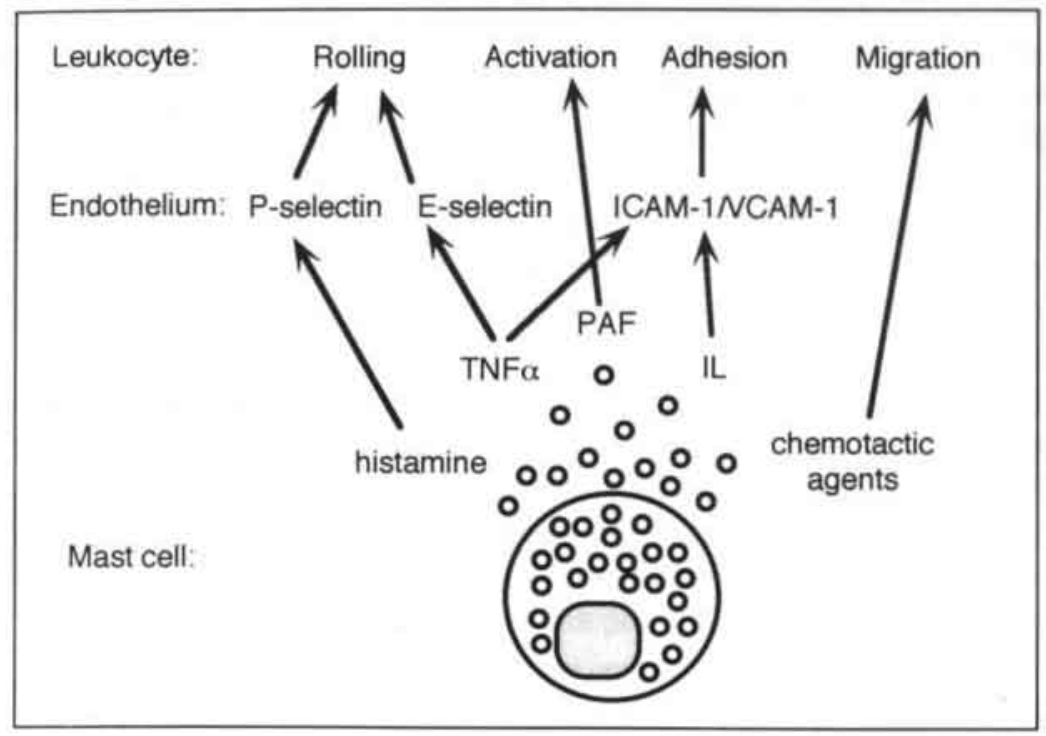

Figure 2.5. Suggested involvement of agents released from mast cells in the various steps leading to leukocyte migration into inflamed tissue. The released agents may stimulate the endothelial cells to express adhesion molecules which may subsequently lead to rolling. activation, adhesion, and, finally, migration of leukocytes.

degranulated mast cells (Jung and Ley, 1997). Secondly, mediators released from mast cells, like PAF, can activate rolling leukocytes (see paragraph 2.3.3). Thirdly, leukocyte adhesion may be increased by various mast cell derived cytokines, like interleukins and TNF $\alpha$, that can stimulate the endothelial cells to express or increase the expression of adhesion molecules. Indeed, it has been shown that degranulation of mast cells induces VCAM-1 and ICAM-1 expression on cultured endothelial cells (Meng et al., 1995; van Haaster et al., 1997). In vivo, leukocyte adhesion induced by a mast cell degranulator could be reduced by a PAF-receptor antagonist (Gaboury et al., 1995). Finally, for the movement of leukocytes into the surrounding tissue chemotactic agents are needed; mast cells are able to release such agents (Springer, 1994). In hamster cheek pouch, for example, induction of degranulation of mast cells with compound $48 / 80$ results in migration of leukocytes from the venules in the direction of the degranulated mast cells, which are mainly situated around the arterioles (Raud et al., 1989b). In summary, mast cells appear to be important cells in the regulation of several steps of the inflammatory reaction of leukocytes and endothelium.

\section{Thromboembolism}

Leukocyte-endothelium interactions can also be modulated by platelet-vessel wall interactions: previous experiments in our laboratory indicate that in the exteriorized 
mesentery of the rabbit leukocyte rolling in venules is influenced by a thromboembolic reaction induced in these vessels (oude Egbrink et al., 1992). The results of this study are summarized in Figure 2.6. Figure 2,6.1 schematically shows a venule with rolling leukocytes. At the site of the arrow the vessel wall was punctured with a glass micropipet to induce a mechanical vessel wall injury. Immediately after puncture the formation of a thrombus started in all vessels. The mere formation of a thrombus by activated blood platelets did not influence leukocyte rolling (Figure 2.6.2). In venules in which adhesion of circulating platelets to the thrombus and subsequent embolization occurred (Figure 2.6.3), the level of leukocyte rolling decreased significantly from the upstream to the downstream vessel segment (median decrease $45 \% ; p \leq 0.001$ ). Inhibition of prostaglandin formation with aspirin $(100 \mathrm{mg} / \mathrm{kg})$ almost completely abolished the influence of the thromboembolic reaction on leukocyte rolling (Figure 2.6.4), but blockade of thromboxane $A_{2}$ receptors with sulotroban ( $30 \mathrm{mg} / \mathrm{kg}$ ) had no such effect. These findings suggest that substances produced by activated platelets and/or damaged vascular cells diminish leukocyte rolling. The identity of these substances is not yet clear, but prostaglandins other than thromboxane $A_{2}$ seem to be involved.

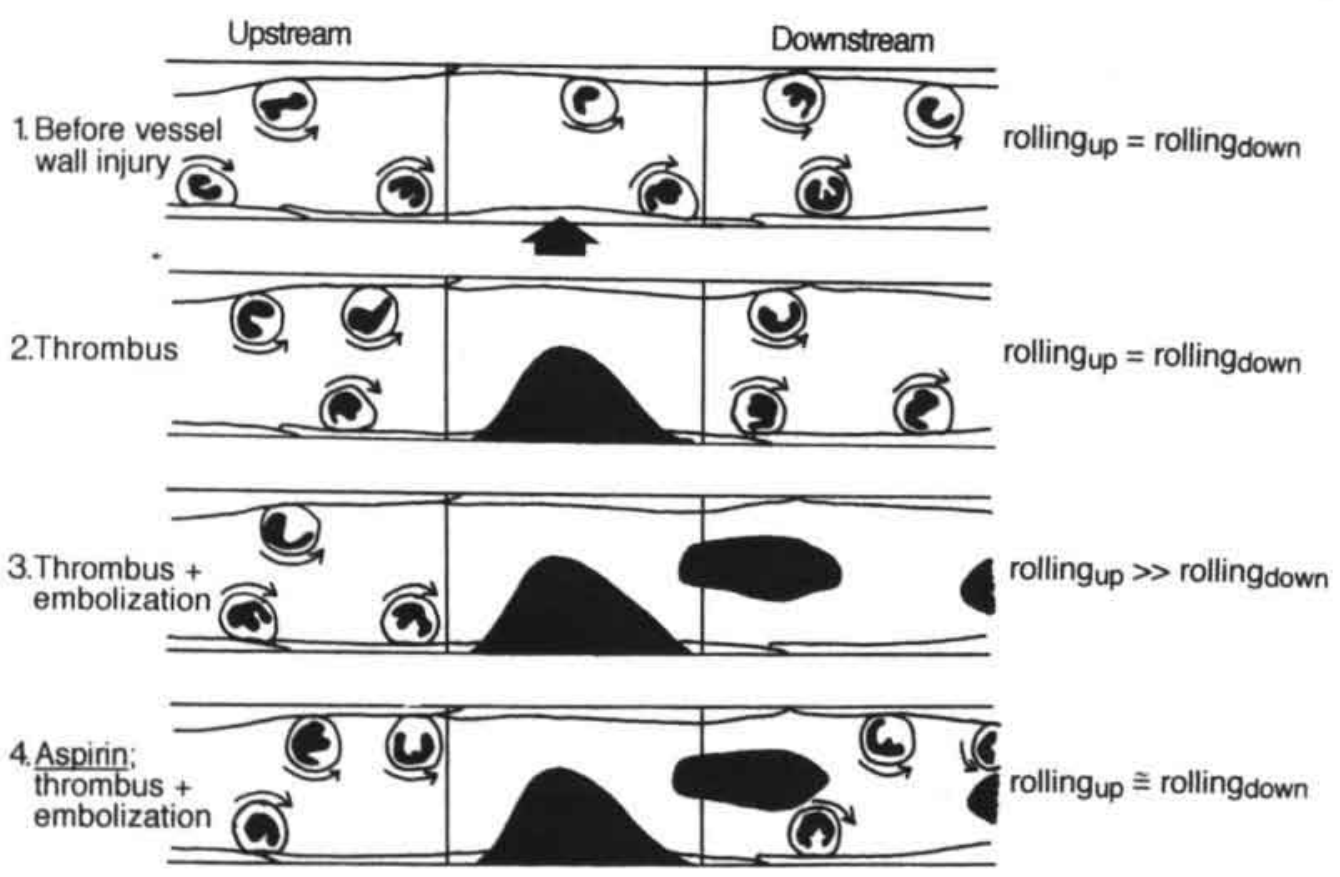

Figure 2.6. Influence of vessel wall injury on leukocyte rolling (oude Egbrink et al., 1992) 1: Venule with rolling leukocytes. At the site of the arrow the vessel wall will be punctured with a glass micropipette to induce an injury. 2: The mere formation of a thrombus following vessel wall injury does not influence leukocyte rolling. 3: If a thromboembolic reaction occurs, the level of leukocyte rolling decreases from the upstream to the downstream vessel segment. 4: Administration of aspirin attenuates the influence of the thromboembolic reaction on leukocyte rolling. 
Other interactions between platelets, endothelium and leukocytes can be divided into two types: adhesion between platelets and leukocytes in flowing blood (a form of aggregation or satellitism, with platelets attached to the surface of leukocytes), and adhesion between platelets and the endothelium, with leukocytes binding to or rolling over the platelets (Nash et al., 1996). These interactions and their possible role in various pathophysiological situations are more and more recognized (Faint, 1992; Nash et al., 1996; Celi et al., 1997).

\section{Prostaglandins}

Prostaglandins (PGs) derive their name from the fact that they were first identified as lipid factors in seminal fluid (thought to be secreted by the prostate gland), which could contract uterine smooth muscle (Gerritsen, 1996). PGs are metabolites of arachidonic acid which, upon activation of a cell, is freed from phospholipid in the plasma membrane by phospholipase $\mathrm{A}_{2}$ or indirectly via phospholipase $\mathrm{C}$. Subsequently, arachidonic acid can be oxygenated by the enzymes cyclooxygenase or lipoxygenase (see Figure 2.7). The cyclooxygenase pathway leads to the production of the following PGs: $P_{G_{2}}, P E_{2}$, $\mathrm{PGF}_{2 \mathrm{a}}$, and $\mathrm{PGI}_{2}$. Thromboxane $\mathrm{A}_{2}\left(\mathrm{TXA}_{2}\right)$ is a product of the cyclooxygenase pathway as well. A fatty acid other than arachidonic acid can be converted to metabolites closely related to PGs such as PGE . $_{1}$ PGs are not stored, but synthesized when they are needed. In the (micro)circulation vascular cells (endothelium, smooth muscle), blood cells (leukocytes, platelets) as well as interstitial cells (mast cells) possess the ability to produce PGs (Gerritsen, 1996).

Two paradoxal theories about the overall role of PGs in inflammation have been proposed: a) PGs are proinflammatory, since inhibition of PG production by aspirin or other non-steroidal antiinflammatory drugs (NSAIDs) diminishes features of inflammation, and b) PGs are antiinflammatory, since their administration in various

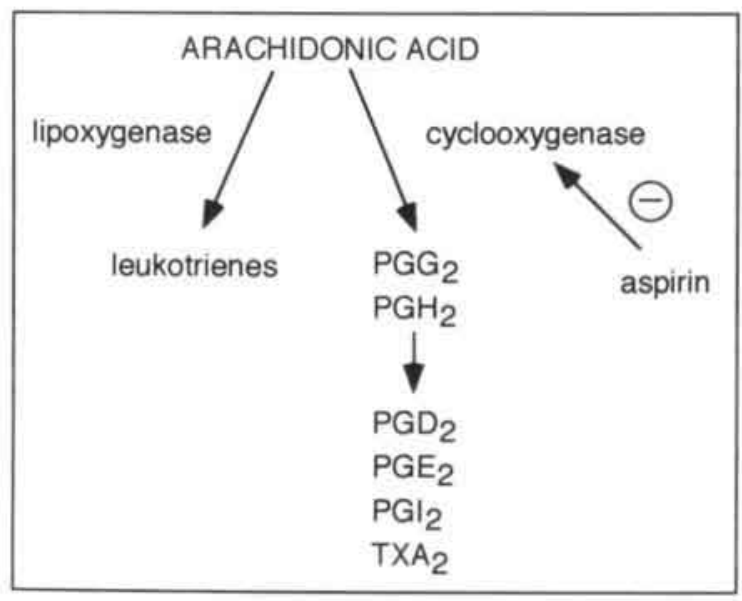

Figure 2.7. Formation of prostaglandins from arachidonic acid via the cyclooxygenase pathway, which is inhibited by aspirin (acetyl salicylic acid). The lipoxygenase pathway lead, among others, to the production of leukotrienes. 
diseases decreases inflammation (Goodwin, 1991; Kitsis et al., 1991). The proinflammatory properties of certain PGs that have been identified, however, are modest and cannot explain the substantial effects of NSAIDs (Goodwin, 1991). In many clinical investigations PGs have been reported to be antiinflammatory and beneficial in many diseases, such as severe chronic heart failure, myocardial ischaemia, and peripheral vascular diseases (see for example Grant and Goa, 1992; Altstaedt et al., 1993; Wigley et al., 1994; Belch et al., 1995; Pacher et al., 1997; Scheeren and Radermacher, 1997). Because PGs have several functions, the exact mechanism behind the beneficial effects is not always clear.

The most important functions of PGs in the microcirculation are regulation of blood flow and permeability, control of hemostasis, and modulation of leukocyte functions (Kaley et al., 1985; Bates, 1995; Gerritsen, 1996). The influences of PGs on leukocyte functions have been extensively studied both in vitro and in vivo; table 2.4 summarizes some of the in vivo studies. It is clear that the effect on leukocyte-endothelium interactions is dependent on the type of PG used and possibly also on the specific situation and tissue under study. In the majority of these studies, however, PGE, and $\mathrm{PGI}_{2}$ seem to inhibit leukocyte-endothelium interactions and leukocyte infiltration into tissues, whereas the $\mathrm{PGE}_{2}$ effect is less clear.

Table 2.4. In vivo effects of various prostaglandins on leukocyte-endothelium interactions or leukocyte infiltration into tissue.

\begin{tabular}{|c|c|c|}
\hline$\overline{P G}$ & effect & reference \\
\hline$\overline{P G E}_{1}$ & rolling/adhesion induced in rat mesentery & Müller et al., 1988 \\
\hline $\mathrm{PGE}_{1}$ & I adhesion following $\mathrm{I} R$ in rat liver & Natori et al., 1997 \\
\hline $\mathrm{PGE}_{1}$ & I infiltration following $1 / R$ in dog myocardium/skin lesions & Simpson et al., 1988 \\
\hline $\mathrm{PGE}_{1}$ & $t$ infiltration into rabbit tear fluid & Srinivasan and Kulkami, 1980 \\
\hline $\mathrm{PGE}_{2}$ & infiltration in rabbit cervix & El Maradny et al., 1996 \\
\hline $\mathrm{PGE}_{2}$ & $=$ rolling in hamster cheek pouch & Higgs et al., 1978 \\
\hline $\mathrm{PGE}_{2}$ & $t$ infiltration into rabbit tear fluid & Srinivasan and Kulkami, 1980 \\
\hline $\mathrm{PGE}_{2}$ & $=$ rolling/adhesion in rat mesentery & Yamaki et al., 1998 \\
\hline $\mathrm{PGI}_{2}$ & I rolling in hamster cheek pouch & Higgs et al., 1978 \\
\hline $\mathrm{PGI}_{2}$ & I infiltration following $\mathrm{l} / \mathrm{R}$ in pig myocardium & Hohlfeld et al., 1993 \\
\hline $\mathrm{PGI}_{2}$ & adhesion induced in rat mesentery & Jones and Hurley, 1984 \\
\hline $\mathrm{PGI}_{2}$ & I infiltration following $\mathrm{I} R$ in rat myocardium & Kanayama et al., 1993 \\
\hline $\mathrm{PGI}_{2}$ & $=$ infiltration following $\mathrm{I} / \mathrm{R}$ in dog muscle & Mohan et al. ,1992 \\
\hline $\mathrm{PGI}_{2}$ & I rolling/adhesion induced in rat mesentery & Müller et al., 1988 \\
\hline $\mathrm{PGI}_{2}$ & I infiltration following $\mathrm{I} R$ in dog myocardium & Simpson et al., 1987 \\
\hline $\mathrm{PGI}_{2}$ & I infiltration into rabbit tear fluid & Srinivasan and Kulkami, 1980 \\
\hline $\mathrm{PGI}_{2}$ & infiltration in damaged spinal cord in rat & Taoka et al., 1997 \\
\hline $\mathrm{PGI}_{2}$ & I rolling/adhesion following $\mid / R$ in rat muscle & Thomson et al., 1994 \\
\hline
\end{tabular}




\section{Angiogenic fators}

Another group of factors or substances with the ability to modulate leukocyteendothelium interactions, is produced in tumors. In animal models it has been observed that, in general, leukocyte rolling as well as adhesion are diminished in tumor microvessels (see Table 2.5). The mechanism behind this observation is not yet elucidated. Two possible causes for diminished leukocyte-endothelium interactions in tumor microvessels have been described. Firstly, it has been shown that less leukocytes are delivered to the newly formed tumor vessels, probably due to a decrease in blood flow in the more tortuous angiogenic vessels (Dellian et al., 1996; Jain et al., 1996). Secondly, the endothelial expression of adhesion molecules appears to be much lower in tumor vessels than in normal vessels (Piali et al., 1995; Griffioen, 1997).

Two important angiogenic factors able to influence leukocyte-endothelium interactions are produced in tumors: basic fibroblast growth factor (bFGF) and vascular endothelial cell growth factor (VEGF) (reviewed in Mason, 1994; Amoroso et al., 1997). In vitro, it has been shown that bFGF reduces the expression of ICAM-1, ICAM-2 and CD34 on HUVECs (Griffioen et al., 1996b). Furthermore, it was demonstrated that the upregulation of ICAM-1, VCAM-1 and E-selectin upon stimulation with inflammatory cytokines, such as TNF $\alpha$ or IL-1, was suppressed by bFGF. In these experiments vascular endothelial cell growth factor (VEGF) had similar, but less pronounced effects (Griffioen et al., 1996a; Griffioen et al., 1996b). The in vivo effects of bFGF and VEGF on leukocyte endothelial interactions are less clear. BFGF has been shown to inhibit leukocyte adhesion (Melder et al., 1996), whereas stimulating (Detmar et al., 1998) as well as inhibiting (Scalia et al., 1999) effects of VEGF on leukocyte adhesion have been reported. 
Table 2.5. Leukocyte rolling and adhesion in venules in normal tissue and in tumor tissue (Griffioen el al., 1998).

Leukocyle rolling Leukocyle adhesion

Tissue

dorsal skinfold chamber in Fischer rats:

rat mammary adenocarcinoma ${ }^{1}$ (Wu et al. 1992)

dorsal skinfold chamber in Fischer rats:

rat mammary adenocarcinoma ${ }^{1}$ (Wu et al, 1994)

dorsal skinfold chamber in Syrian Golden ham-

sters: amelanotic melanoma (Dellian et al, 1995)

dorsal skinfold chamber in $\mathrm{C} 3 \mathrm{H}$ mice ${ }^{2} /$

dorsal skinfold chamber in SCID micez:

murine mammary adenocarcinoma;

cranial window in $\mathrm{C} 3 \mathrm{H}$ mice 1 :

murine mammary adenocarcinoma:

cranial window in SCID mice:

murine mammary adenocarcinoma'/

human glioblastoma' (Fukumura of al., 1995)

dorsal skinfold chamber in nude mice:

Lewis lung carcinoma (Borgstróm et al., 1997)

Means and SEM are presented; "statistically significant difference of lumor venules versus normal venules reported;

'data extracted from figure; '2 data extracted pantly from text, partly from figure; leukocyle rolling is expressed as number of cells per

minute, or as the rolling fraction of all passing leukocytes; leukocyte adhesion is expressed as number of adherent cells per surface area

normal

$5.8 \pm 1.8 / \mathrm{min}$

tumor

$100 \pm 30 / \mathrm{mm}^{2}$

tumor

$16.0 \pm 3.0 / \mathrm{min} \quad 0.6 \pm 0.2 / \mathrm{min}^{\circ} \quad 35 \pm 12 / \mathrm{mm}^{2} \quad 19 \pm 12 / \mathrm{mm}^{2} \cdot$
$63.3 \pm 9.9 \%$

$43 \pm 24 \%$

$29 \pm 16 \%$

$23+12 / \mathrm{min}$
$29 \pm 16 \%$
$14 \pm 4 \%$
$28+21 \%$

$9 \pm 7 \%$

$34 \pm 40 \%$

$11 \pm 14 \%$
$31 \pm 9 / \mathrm{mm}^{2}$

$136.7 \pm 110.9 / \mathrm{mm}^{2}$

$42.2 \pm 4.8 / \mathrm{mm}^{2}$

$103 \pm 72 / \mathrm{mm}^{2}$

$82 \pm 92 / \mathrm{mm}^{2}$

$82 \pm 92 / \mathrm{mm}^{2}$

$185 \pm 113 / \mathrm{mm}^{2}$ $10 \pm 0 / \mathrm{mm}^{2}$
$52 \pm 0 / \mathrm{mm}^{2}$ $186 \pm 176 / \mathrm{mm}^{2}$

$0 / \mathrm{mm}^{2}$ 
In this chapter an overview is presented of the materials and methods used in the studies described in this thesis. Emphasis is given to the basis underlying the choices we have made for quantitation and presentation of leukocyte-endothelium interactions.

\subsection{Animals and experimental setup}

Animal handling was performed according to the Dutch Law on Animal Experimentation (WOD) and The European Directive for the Protection of Vertebrate Animals used for Experimental and other Scientific Purposes (86/609/EU). The protocols were approved by the Animal Experimental Committee of Maastricht University, The Netherlands.

\section{Animals}

Experiments were performed on 3 different species. New Zealand white rabbits of either sex were used in the chapters $4,5,7$, and 8 , since most of these chapters were based on studies previously performed on the same species. Male rats of various strains were used in chapter 6 to study the involvement of mast cells; this could not be done in rabbit mesentery in which hardly any mast cells are present. Male Swiss mice were used in chapter 9 , because in our institution experience existed with regard to the effects of angiogenic factors in this species.

All animals were housed in the central animal facilities and had ad libitum access to water and food. For detailed information about the animals, the anesthetic and surgical procedures followed and the setup of the different experiments, the reader is referred to the various chapters.

\section{Intravital microscopy}

In rabbits and rats the mesentery was used for microscopic observation; this tissue can be exposed without the need of extensive surgery, it is thin and transparent and, therefore, well suited to study leukocyte-endothelium interactions. In mice the cremaster muscle was employed, since the scrotum in which the cremaster muscle is situated, is very suitable for the local insertion of pellets or injection of agents. In this way, small amounts of expensive agents can be locally applied in the direct environment of the vessels under investigation. Microvessels in these tissues were visualized in situ using a Leitz intravital microscope, adapted for telescopic imaging (Slaaf et al., 1982), and a Leitz water-immersion objective (25x). Transillumination was performed with a tungsten lamp. Images were recorded on videotape through either a TV camera or a CCD camera. Final magnification at the front plane of the camera was 52x. For inspection of the preparation and mapping of the microvasculature, a low magnification objective $(4 x)$ was employed.

\section{Mesentery}

In rabbits and rats the mesentery is a thin and transparent layer of peritoneum (see Figure 3.1). It connects the small intestine to the posterior abdominal wall and is 


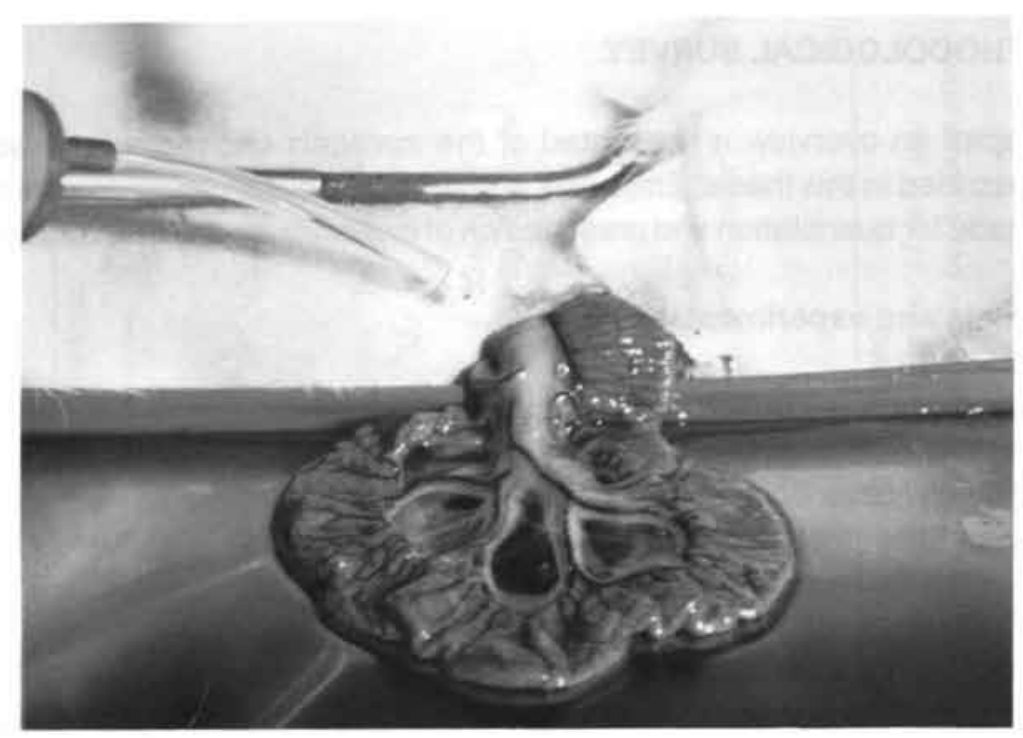

Figure 3.1. Exposed rabbit mesentery (thanks to B. Woldhuis).

composed of loose connective tissue containing vessels and nerves passing to and from the bowel. For microscopic observation a segment of the distal ileum was exteriorized through a small abdominal incision. The mesentery was then carefully spread over a siliconized glass plate mounted in an electrically heated table of an intravital microscope. The tissue was continuously superfused with a buffered Tyrode's solution (composition: $11 \mathrm{mM}$ glucose, $13 \mathrm{mM}$ sucrose, $130 \mathrm{mM} \mathrm{NaCl}, 5.6 \mathrm{mM} \mathrm{KCl}, 2.9$ $\mathrm{mM} \mathrm{CaCl}_{2}, 1.2 \mathrm{mM} \mathrm{MgCl}_{2}, 25 \mathrm{mM} \mathrm{NaHCO}_{3}$, and $1.4 \mathrm{mM} \mathrm{NaH}_{2} \mathrm{PO}_{4}$ ) at $37^{\circ} \mathrm{C}$, saturated with a mixture of $\mathrm{N}_{2}(95 \%)$ and $\mathrm{CO}_{2}(5 \%)$. The bowels were kept moist with overlying wet gauze.

After handling the mesentery, the tissue was allowed to stabilize. To determine the optimal length for a stabilization period, levels of leukocyte rolling were determined in three rabbits (11 venules) during a period of 80 minutes in which no intervention had taken place. The results are shown in Figure 3.2. It is clear that in the first 30 minutes following exteriorization the level of leukocyte rolling decreased in most venules. In the venules in which more than 50 leukocytes were rolling 30 minutes following exteriorization, levels of leukocyte rolling continued to decrease or strongly fluctuated. In the other vessels levels remained rather stable. Therefore, we decided to allow the tissue to stabilize for 30 minutes before baseline recordings were made. In those studies in which no experimental intervention was applied before baseline recordings, only venules with a baseline level of 50 or less rolling leukocytes per minute at 30 minutes following exteriorization were included.

In order to be able to accurately detect decreases in the level of leukocyte rolling over time or as induced by the substances of interest, only venules with a minimal level of 5 to 10 rolling leukocytes per minute were included in the studies. Hardly any mesenteric venule had to be excluded. 


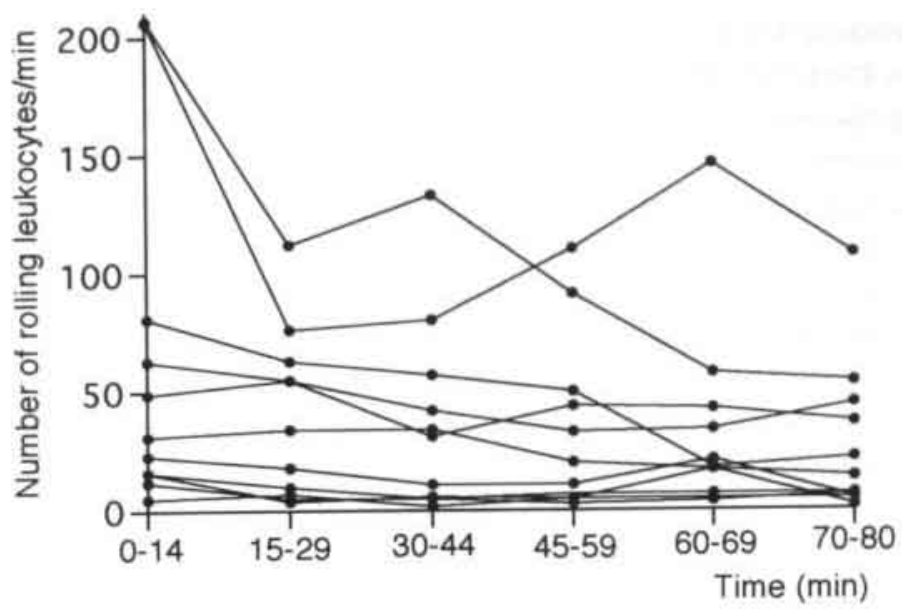

Figure 3.2. Levels of leukocyte rolling in 11 venules at different periods following mesentery exteriorization in 3 rabbits.

\section{Cremaster muscle}

The cremaster muscle is a sack of striated muscle, the contraction of which causes the testis and epididymis to be pulled towards the abdomen. For intravital microscopy the right cremaster muscle sack was opened and the testis and epididymis were advanced into the abdominal cavity. The muscle was spread out with the help of sutures at its periphery and continuously superfused with Krebs' solution (composition: $25 \mathrm{mM}$ $\mathrm{NaHCO}_{3}, 1.2 \mathrm{mM} \mathrm{KH}_{2} \mathrm{PO}_{4}, 118.4 \mathrm{mM} \mathrm{NaCl}, 4.8 \mathrm{mM} \mathrm{KCl}, 2.5 \mathrm{mM} \mathrm{CaCl}_{2}, 1.2 \mathrm{mM} \mathrm{MgSO}_{4}$ ) at $34-36^{\circ} \mathrm{C}$, saturated with a mixture of $\mathrm{O}_{2}(95 \%)$ and $\mathrm{CO}_{2}(5 \%)$. The muscle was covered with clear plastic wrap to prevent dehydration and effects of $\mathrm{O}_{2}$ in the air on fluid dynamic parameters. After handling the cremaster muscle the tissue was allowed to stabilize for 30 minutes, before the experiments started.

\section{Systemic leukocyte counts}

To elucidate a possible influence of the experimental procedure on circulating leukocytes, the systemic number of leukocytes was determined at the beginning and at the end of each experiment. In some studies an additional count was performed at some instant during the experiment. Leukocytes were counted and differentiated as polymorphonuclear (PMN) or monomorphonuclear (MMN) in a counting chamber. Of these two leukocyte subclasses, the PMNs are the ones that are predominantly rolling in venules of the rabbit mesentery (Tangelder et al., 1995).

\subsection{Assessment of microvascular hemodynamic parameters}

\section{Red blood cell velocity}

For on-line measurement of red blood cell (RBC) velocity in microvessels a bidirectional optical (BDO) three-stage prism grating system was used (Slaaf et al., 1981; Slaaf et al., 1984). The microscopic image of the red blood cells is projected onto a grating 
placed transversely to the direction of flow. The light modulated by the moving red blood cells is split by the prism grating into three directions, resulting in three signals detected by three photosensors (spatial filtering). The velocity of the red blood cells can be computed from the frequency of the signal (inversely related to the time between two successive appearances on one and the same photosensor), the optical magnification and the grating constant (Slaaf et al., 1981; Slaaf et al., 1993). For each vessel the sensor width was adjusted to cover the full width of the vessel in order to assess a measure for mean RBC velocity ( $\left.v_{\text {men }}\right)$ over the cross-sectional area; care was taken to ensure that the vessels remained within the sampling area during lateral tissue movements. To obtain actual mean $\mathrm{RBC}$ velocities, the measured velocity values were divided by a conversion factor of 1.1 (Tangelder et al., 1986).

\section{Diameter}

Diameters of the venules and arterioles under investigation were measured off-line, with the use of a home-built video image shearing device (according to Intaglietta and Tompkins, 1973). Care was taken to measure the diameter with focus on the widest part of the vessel and to measure the same vessel site when a vessel was to be measured repeatedly.

\section{Reduced velocity}

The wear and tear exerted on endothelial cells by the blood is related to the shear rate at the vessel wall. In Figure 3.3 the concept of shear rate is elucidated. Two adjacent layers of fluid are depicted schematically. They differ in velocity by $\Delta v$. The velocity gradient $(d v / d x)$, expressed in $s^{-1}$, is called shear rate. The shear rate varies across a microvessel (it is zero in the center and highest at the wall) and depends on the actual velocity profile (Slaaf et al., 1993).

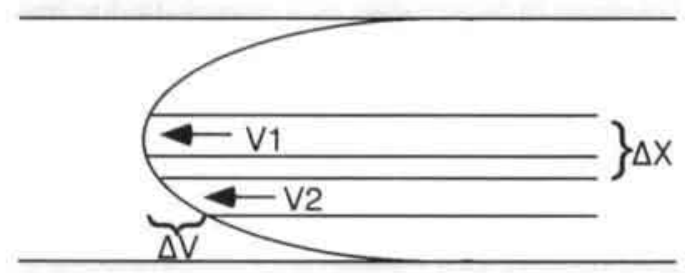

Figure 3.3. Schematic presentation of the concept of shear rate, i.e., the velocity gradient between two adjacent fluid layers. Longitudinal section of a cylindrical tube showing a velocity profile and two adjacent layers of a fluid. 
In case of a Newtonian fluid, the velocity profile is parabolic; the shear rate increases linearly from the center towards the wall. In this case shear rate at the wall of a vessel, called wall shear rate $\left(W S R_{\text {pan }}\right.$ for a parabolic velocity profile), is given by:

$$
W S R_{\text {par }}=4 \frac{v_{\text {mean }}}{r}
$$

However, since blood is a non-Newtonian fluid, velocity profiles in microvessels are not parabolic. In arterioles, the velocity profile was shown to be flattened as compared to a parabola (Tangelder et al., 1986). As a consequence, wall shear rates in these microvessels appeared to be about 2.1 times higher than expected on the basis of a parabolic velocity distribution with the same volume flow (Tangelder et al., 1988). In venules, velocity profiles are also blunted as compared to a parabola (Tangelder and colleagues, unpublished observations). Therefore, also in venules $W S R_{p a r}$ has to be multiplied by a certain correction factor $(\alpha)$ to obtain estimates of the actual wall shear rate $W S R_{\text {ver }}$. Assuming that the velocity profile is invariant under various conditions in one vessel, $W S R_{\text {ven }}$ can be expressed as:

$$
W S R_{\text {ven }}=\alpha \frac{v_{\text {mean }}}{r}
$$

In this thesis, reduced velocity, which is defined as $v_{\text {mean }} / 2 r$, is used as a measure of wall shear rate.

\subsection{Evaluation of leukocyte-endothelium interactions}

A number of parameters can be used to evaluate leukocyte-endothelium interactions. First, we will describe the parameters employed to assess leukocyte rolling and adhesion as described in the literature; subsequently, we will elaborate on the parameters used in this thesis.

\section{Leukocyte rolling and adhesion: literature}

Leukocyte rolling is mostly presented as the number of rolling leukocytes passing a venular segment per unit of time, often called rolling leukocyte flux and expressed as number of rolling cells per minute (see for example: von Andrian et al., 1991; oude Egbrink et al., 1992; Ley, 1994), per 30 seconds (Wu et al., 1992) or per second (Asako et al., 1994).

Some studies present the leukocyte rolling fraction, defined as the percentage of the total number of passing leukocytes that is rolling. To this purpose, all leukocytes are fluorescently stained with, for example, acridine red (Ley and Gaehtgens, 1991; von Andrian et al., 1991) or rhodamine 6 (Nolte et al., 1994). Both freely flowing and rolling leukocytes are observed with (stroboscopic) epi-illumination. The total number of fluorescent cells passing a venular segment is counted and, subsequently, the number of rolling cells in the same segment is determined. The rolling fraction is then calculated 
as the number of rolling leukocytes per 100 leukocytes passing the segment (Ley and Gaehtgens, 1991; von Andrian et al., 1993b). The disadvantage of this method is that a fluorescent dye has to be used to visualize the free flowing leukocytes; such a dye may influence cell functioning. Although it has been demonstrated that the fluorescent dyes used in these studies do not influence systemic leukocyte counts, the interactions of leukocytes with the vessel wall, and microvascular hemodynamics (Ley and Gaehtgens, 1991; von Andrian et al., 1991; Janssen et al., 1994), we prefer not to use such dyes when transparent tissues like mesentery and cremaster muscle are used in which rolling cells can be observed clearly.

An approximation of the leukocyte rolling fraction may be obtained without the use of fluorescent dyes, by estimating the flow in a microvessel and assuming that the systemic concentration of leukocytes is maintained throughout the circulation. Flow in a microvessel is calculated from the mean RBC velocity and the vessel radius $(r)$ : $V_{\text {mean }} \pi r^{2}$. The systemic number of leukocytes per $\mathrm{ml}$ blood is measured in blood obtained from a central ear artery (see paragraph 3.1). Using these two parameters the total number of leukocytes passing the venular segment of observation can be estimated. Combined with the number of rolling cells per minute this information provides an estimate of the rolling fraction.

Taking into account that in mesentery nearly all rolling leukocytes are polymorphonuclear cells (PMNs; Tangelder et al., 1995), the rate of supply of PMNs by the blood stream can be obtained when the total number of leukocytes passing the venular segment is multiplied by the systemic PMN fraction. Then the rolling fraction of PMNs can be calculated. If the rolling fraction of PMNs is high, i.e., almost all passing PMNs are rolling, a possible influence of an agent may be masked by the fact that no more PMNs can be recruited to start rolling.

Leukocyte rolling velocity can be determined from the time required for individual leukocytes to roll along a given distance of the venule (von Andrian et al., 1991; Asako et al., 1994; Janssen et al., 1994; Kubes and Kanwar, 1994), or by measuring the distance that was covered between two or more successive video frames (Ley and Gaehtgens, 1991).

In some studies the number of rolling leukocytes per $100 \mu \mathrm{m}$ vessel length was calculated by dividing leukocyte flux by leukocyte rolling velocity (Asako et al., 1994; Kubes and Kanwar, 1994). This parameter can be considered as a measure of the rolling leukocyte density; this implicates that not only the number of rolling cells but also their transittime is taken into account. A similar parameter is the relative rolling index (Thorlacius et al., 1994), calculated as the leukocyte rolling fraction divided by the rolling velocity. This parameter has also been used to reveal the influence of an intervention on rolling fraction and rolling velocity together.

Leukocytes are considered to be adherent (sticking) when they remain stationary for a period equal to or greater than 30 seconds (von Andrian et al., 1992; Kubes et al., 1993; Beuk et al., 1997). Leukocyte adhesion can be expressed as the number of adherent cells per vessel length or per endothelial surface area.

An overall parameter that includes the number of rolling leukocytes and their velocity, and the number of adherent leukocytes is the total number of leukocytes interacting with 
the wall in a certain vessel segment at a particular moment (Raud et al., 1988; Beuk et al., 1997). In transparent tissues, without the use of a fluorescent dye, this parameter can be determined by counting the number of visible (i.e., interacting with the endothelium) leukocytes within a $100 \mu \mathrm{m}$ vessel segment in a frozen video frame.

For the studies described in the present thesis, we determined the level of leukocyte rolling (identical to leukocyte rolling flux, see above), the leukocyte rolling velocity, as well as the number of adherent leukocytes. Details about the way these parameters were assessed are given below.

\section{Assessment of the level of leukocyte rolling}

The level of leukocyte rolling is quantitated off-line by counting the cells that roll or saltate through an unbranched segment of a venule; the length of the segment is approximately equal to the diameter of the venule. The level of leukocyte rolling is expressed as the number of rolling and saltating leukocytes per minute.

To get more insight into the relative number of saltating and rolling leukocytes, we counted in a sample of venules the number of leukocytes passing the predefined segment of the venule: those leukocytes that stayed in contact with the vessel wall in the whole segment were defined to be rolling, and those that were not in contact with the vessel wall in the whole segment were called saltating. It appeared that of all these passing cells the percentage of rolling cells was $96 \%$ (median; range $81-100 \%$ ). A median of only 1 saltating cell per minute was found (range $0-5 / \mathrm{min}$ ).

Figure 3.4 shows the cumulative number of rolling leukocytes counted in a vessel segment during 10 minutes, i.e., the total number of rolling leukocytes counted between the start of the experiment and the moment indicated on the $x$-axis. The slope of the curve is a measure of the level of leukocyte rolling. It is clear that the level of leukocyte rolling varies with time and that the mean level of leukocyte rolling depends on the period of averaging. The shorter the period of averaging is, the larger the variations over the 10 minutes period will be. By inspecting the curve, one notes that for instance a counting period of 30 seconds will lead to a high variability in the levels of leukocyte rolling: compare a start at 0 with one at 100 seconds. An average over the whole period of 10 minutes provides the best measure of the mean level of leukocyte rolling. However, the protocols of the studies described in this thesis do not allow the recording of periods of more than 2 minutes. We used 3 recordings of 10 minutes to study the variability of leukocyte rolling. In each cumulative distribution, we divided the sequence into $2,4,6,8,12$, and 24 equal periods $(300,150,100,75,50$, and 25 seconds, respectively) and determined during each recording the relative dispersion (also called coefficient of variation: standard deviation/mean of the counts). In all three recordings of 10 minutes the relative dispersion of averaging over a period of at least 100 seconds was less than $20 \%$; the higher the level of leukocyte rolling was, the lower the relative dispersion. Since the relative dispersion of the level of leukocyte rolling of groups of vessels is $50-100 \%$, the variability within measurements is much lower and, therefore, negligible. In our experiments counting periods range from 100 to 120 seconds. 


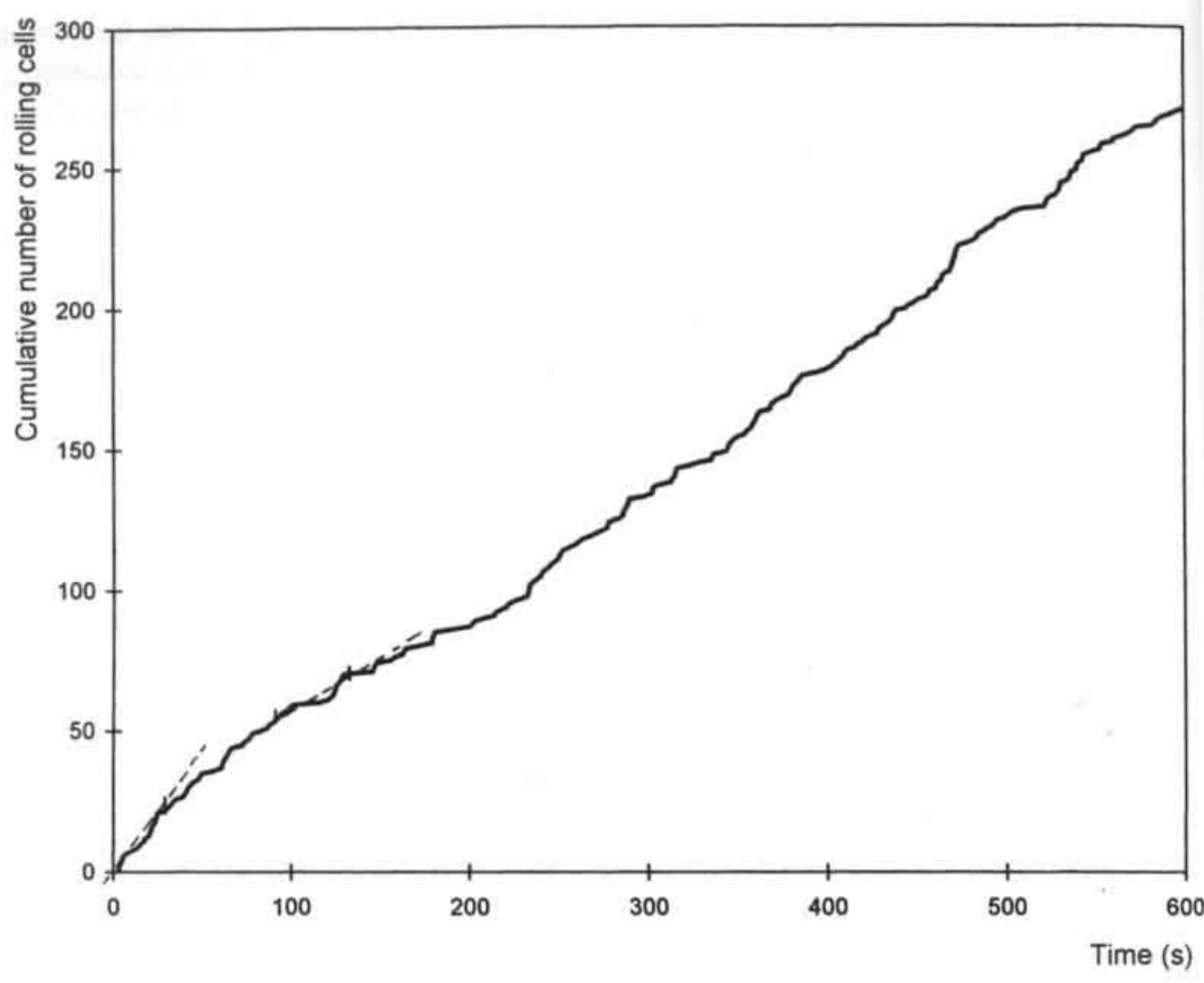

Figure 3.4. Cumulative number of rolling leukocytes counted in one venule during 10 minutes, with average slopes over 30 second periods starting at 0 and at 100 seconds indicated. See text for explanation.

To evaluate the accuracy of the counting technique, five investigators were asked to count rolling leukocytes in taped sequences of the same four venules with levels of leukocyte rolling varying from about 30 to 190 cells per minute. Each investigator performed counts in duplicate with a mean counting period of 110 seconds. If duplicate counts differed by more than $10 \%$, a third count was performed. The average deviation of duplicate counts from their mean average value in the four venules ranged from 4 to $6 \%$ for the various investigators. No relationship between the deviation and the number of rolling leukocytes was found.

Table 3.1 presents the mean levels of rolling leukocytes assessed by the various observers. The variation between the various observers was only 3 to $10 \%$. Therefore, it can be concluded that counting could be performed in an accurate manner. For this thesis, counts were performed mainly by one observer (D), while a control was performed by a second observer $(E)$, who performed a sample of randomly chosen counts. 
Table 3.1. Means from duplicate counts of levels of leukocyte rolling (\#/min) counted in four arbitrary venules by five observers.

\begin{tabular}{lllll}
\hline & \multicolumn{4}{c}{ venule } \\
\cline { 2 - 5 } Observer & 1 & 2 & 3 & 4 \\
\hline A & 31.5 & 82.5 & 113 & 180 \\
B & 37.5 & 94 & 109.5 & 180.5 \\
C & 29.5 & 87 & 117.5 & 192.5 \\
D & 29 & 86 & 122 & 184 \\
E & 32 & 87 & 131 & 193.5 \\
\hline
\end{tabular}

\section{Leukocyte rolling velocity}

Median leukocyte rolling velocity per vessel was determined off-line by measuring the time needed for 10 randomly chosen rolling leukocytes to travel a certain distance along the vessel wall. This distance was about one to three times the diameter of the vessel.

\section{Leukocyte adhesion}

Leukocytes were considered to be adherent when they remained stationary for at least $30 \mathrm{~s}$. The number of adherent leukocytes was determined in a $100 \mu \mathrm{m}$ segment of a venule. The mean number present at any instant was assessed over a period of 2 minutes. In chapter 9 the level of leukocyte adhesion was expressed as the number of adherent cells per endothelial surface area $\left(\right.$ per $\mathrm{mm}^{2}$ ), since the diameters in the various experimental groups were not similar.

\subsection{Statistics and data presentation}

\section{Statistics}

Because of the nonsymmetrical distribution of the majority of the collected data, nonparametric statistic tests were used to compare data groups (Siegel and Castellan, 1988). Paired data groups were compared using the Wilcoxon signed ranks test; for more than two related samples the Friedman two-way analysis of variance was used. Differences between two independent data groups were tested with the Mann-Whitney $U$ test; to compare more than two dependent data groups the Kruskal-Wallis test was used. To find correlations between data groups, the Spearman rank-order correlation coefficient was determined. In all tests, the level of significance was set at 0.05 .

A large variation was found in the various parameters between venules within each animal; this intervenular variation appeared to be similar to the interanimal variation. This may be partly due to the fact that within one animal only venules were selected that were independent of each other for their blood supply or drainage. Furthermore, endothelial functioning may be heterogeneous in different venules, even within one tissue. It is known that vessels exhibit a high level of heterogeneity within the mesenteric microvascular bed (Pries et al., 1995), which may contribute to the heterogeneous effects of interventions. Therefore, we considered it appropriate to perform the statistic tests on parameters measured per venule, rather than per animal. 
Data presentation: medians and interquartile range

Several methods can be used to present data. In most papers in which leukocyteendothelium interactions are studied values are reported as means with standard errors (SE; see for example Kubes et al., 1993; Ley, 1994). Such a representation requires a normal distribution of the data. If mean and SE are used on not normally distributed data, one should realize that they are considerably influenced by outlying observations. For ordinal data and/or data with outliers the median is a better measure of central tendency because it uses ranking information. Data concerning leukocyte-endothelium interactions in vivo are usually distributed nonsymmetrically, and, hence, presentation as medians with interquartile ranges (i.e., the spread from 25 th to 75 th percentile) is more appropriate. In this way $50 \%$ of the data set is represented within bars, and the skewness of the data distribution is preserved. To illustrate differences in the way of presentation two data sets (extracted from chapter 6, Figure 6.2B) are presented in three ways in figure 3.5 : (A) as individual measurements, $(B)$ as medians with interquartile ranges, and $(\mathrm{C})$ as means with standard errors. Comparison of these three ways of presentation makes it clear that medians with interquartile ranges reflect more accurately the distribution of the original data set as compared to means with standard errors. In this thesis data are presented as medians with interquartile ranges, unless indicated otherwise. One has to be aware that this way of data handling entails the presentation of large variation bars, and, therefore, data seem to be more variable as compared to data described in literature where means and standard errors are used.

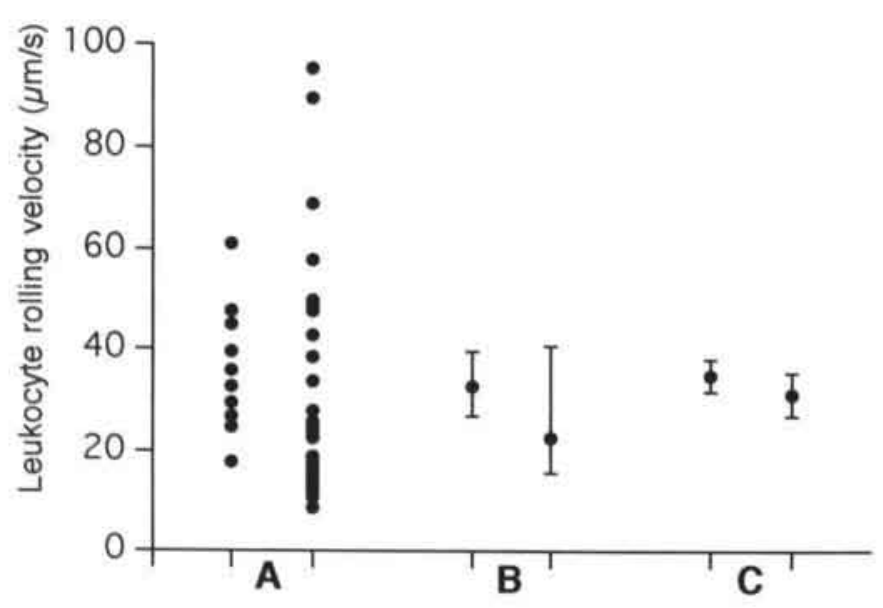

Figure 3.5. Presentation of one data set on the velocity of rolling leukocytes in rabbit mesenteric venules as (A) individual measurements, $(B)$ medians with interquartile ranges, and (C) means with standard errors. 


\section{Data presentation: normalization}

In the studies presented in this thesis, we were interested in changes which occurred in leukocyte-endothelium interactions due to an intervention. Variability in data can be reduced by computing the relative change in, for example, the level of leukocyte rolling. To this purpose, data are normalized to their individual baseline values. In Figure 3.6 this normalization procedure is elucidated. Figure 3.6.A shows for several venules the absolute level of leukocyte rolling at two instants ( $\mathrm{t} 1$ and $\mathrm{t} 2$ ). In Figure 3.6. B changes occuring over time are visualized after normalizing for each individual venule the levels observed at $\mathrm{t} 2$ to those observed at $\mathrm{t} 1$.

If levels of leukocyte rolling decrease with time, the normalized values will be between 1 and 0 , whereas increases in the levels will lead to values from 1 to infinity. This asymmetry implicates that normalization of the levels of leukocyte rolling observed at $\mathrm{t} 1$ to those observed at $\mathrm{t} 2$ (i.e., the reverse procedure as described for Figure 3.6.B) results in a different distribution, shown in Figure 3.6.C. In the non-symmetrical tests used in this thesis and described above, not only the sign of the differences but also their relative magnitude is considered. The Wilcoxon signed ranks test, for example, gives more weight to a pair which exhibits a large difference between the two instants than to a pair which exhibits a small difference (Siegel and Castellan, 1988). Therefore, p-values obtained by testing the values of Figures 3.6. B and C are not similar. This asymmetry can be avoided by computing the logarithm of each value. In this way the normalized values will be negative if the level of leukocyte rolling decreases with time, whereas increases in the levels will lead to positive values (Figure 3.6.D). It is clear from Figure 3.6.E that computing the logarithm of the values of Figure 3.6.C yields a mirror image of Figure 3.6.D (only the signs have changed). Therefore, p-values obtained by testing the values of Figures 3.6.D and $E$ are similar. In this thesis logarithms of normalized values are presented unless otherwise indicated. 

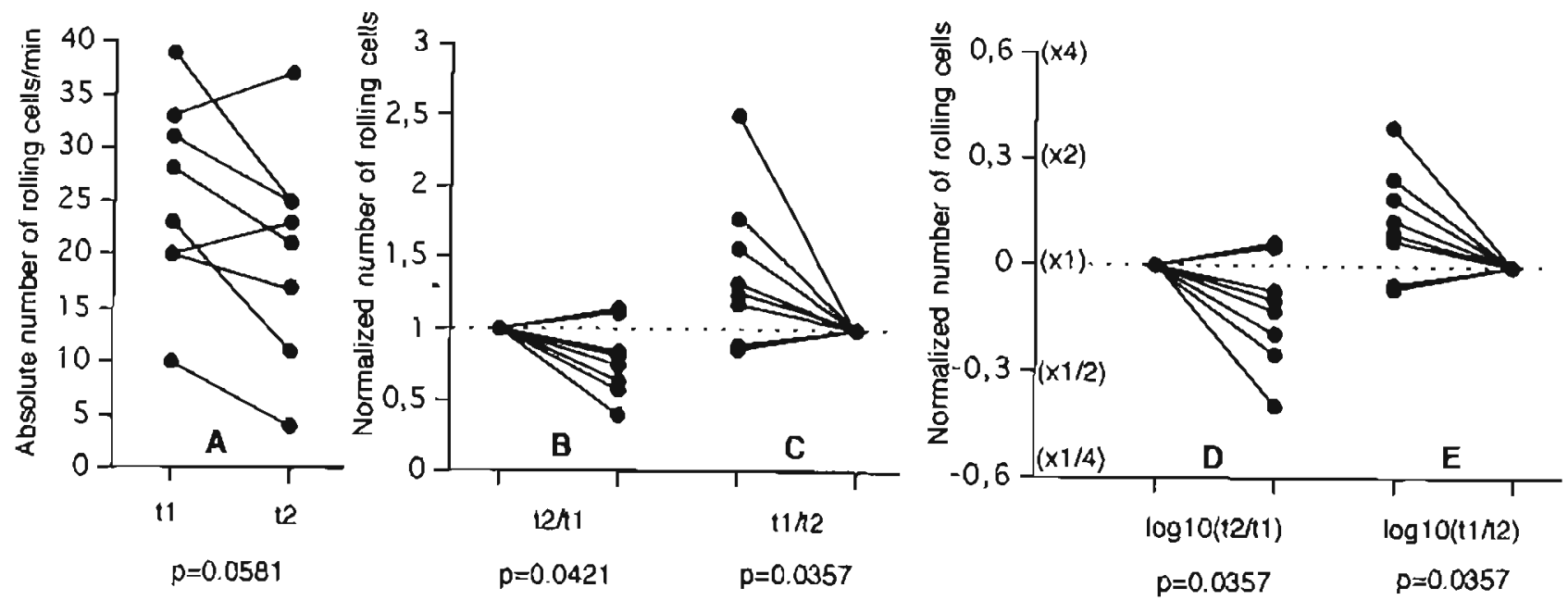

Figure 3.6. Explanation of normalization procedure as exemplified for the levels of leukocyte rolling at two time points in various venules: $(A)$ absolute data, $(B$ and $C$ ) normalized data, and $(D$ and $E$ ) logarithm of normalized data. See text for details.

$\{1, t 2=$ time periods; $p=p$-value obtained by using Wilcoxon signed ranks test 
4 HOW TO ADMINISTER AGENTS TO STUDY THEIR LOCAL EFFECT ON LEUKOCYTE-ENDOTHELIUM INTERACTIONS IN MESENTERIC VENULES

\begin{abstract}
To study local effects, agents are often infused into a small feeding artery or administered topically. Saline is commonly used as the vehicle, and, hence, its infusion or superfusion is employed as control assuming no influence of this procedure itself. When infusing saline into a distal small side-branch of the rabbit cranial mesenteric artery, however, leukocyte-endothelium interactions in mesenteric venules were found to be affected; the number of rolling leukocytes was decreased and leukocyte rolling velocity was significantly increased as compared to superfused saline. This is an unexpected and undesirable finding, that should be kept in mind when planning experiments in which effects of locally administered agents are to be studied.
\end{abstract}




\subsection{Introduction}

Methods of local administration are preferably used to study the effects of substances which are scarce and/or expensive, or which might have detrimental or toxic effects when administered systemically. In our laboratory, methods of local administration are applied to investigate the influence of certain water-soluble agents on leukocyte-vessel wall interactions in rabbit mesenteric venules. To assess whether the route of administration (from the luminal or the abluminal side of the microvasculature) has an influence on the effects of the drugs, substances are either infused through a small distal side-branch of the cranial mesenteric artery, feeding the mesenteric segment under observation, or superfused on the mesentery. In such experiments saline, which serves as vehicle, is infused or superfused as control; effects of the agents under study, if any, are compared to that of saline. This is considered a standard procedure; in general no major effects of the vehicle are expected. To our surprise, however, we observed that local infusion of saline decreased leukocyte-endothelium interactions considerably: the level of leukocyte rolling decreased and leukocyte rolling velocity increased.

Therefore, in the present methodological study the two methods of local administration are compared. To this purpose, the effects of local infusion and of topical administration of saline on leukocyte rolling and on hemodynamic parameters in rabbit mesenteric venules were studied.

\subsection{Materials and methods}

\section{Animal preparation}

All experiments were approved by the local ethical committee on the use of laboratory animals. New Zealand white rabbits of either sex were used $(n=11$; weight 1.6 to $2.5 \mathrm{~kg}$ ). Anesthesia was induced by intramuscular injection of $40 \mathrm{mg} / \mathrm{kg}$ body wt ketamine hydrochloride (Nimatek, Ad Usem Veterinarium, Cuijk, The Netherlands) and $4 \mathrm{mg} / \mathrm{kg}$ body wt xylazine hydrochloride (Sedamun, Ad Usem Veterinarium, Cuijk, The Netherlands). It was maintained by continuous infusion of ketamine (40 $\mathrm{mg} / \mathrm{kg} / \mathrm{h}$ ) and xylazine $(5 \mathrm{mg} / \mathrm{kg} / \mathrm{h}$ ) dissolved in a Lactetrol solution (Aesculaap, Boxtel, The Netherlands; $15 \mathrm{ml} / \mathrm{h}$ ) through a catheter in the femoral vein.

The experimental setup has been described in detail before (oude Egbrink et al., 1992). In short, rabbits were ventilated to maintain systemic arterial $\mathrm{pH}, \mathrm{pCO}_{2}$, and $\mathrm{pO}_{2}$ at normal values. Mean values ( \pm s.d.) were: $\mathrm{pH} 7.48 \pm 0.05, \mathrm{pCO}_{2} 39 \pm 4$ $\mathrm{mmHg}$, and $\mathrm{pO}_{2} 99 \pm 15 \mathrm{mmHg}$. Arterial blood pressure was measured through a catheter in the femoral artery and heart rate was determined from the instantaneous pressure signal. Body temperature was kept between 37 and $38^{\circ} \mathrm{C}$ by means of a servo-controlled infrared heating lamp.

Through an abdominal midline incision, the terminal ileum was exteriorized and carefully spread over a siliconized glass plate on the microscope table. The bowels were kept moist with overlying wet gauze. The mesentery was continuously superfused with buffered Tyrode's solution $\left(37^{\circ} \mathrm{C} ; \mathrm{pH} 7.35-7.40\right)$. With a Leitz 
intravital microscope and a Leitz water-immersion objective (SW25, nummerical aperture 0.60 ) the mesenteric microcirculation was visualized. Transillumination was performed with a tungsten lamp. Video recordings were made through a TV camera (Bosch, Ultricon 4532, 1 inch).

In 4 rabbits (the infusion group) a side-branch of the small artery feeding the microvessels within the mesenteric segment under observation was retrogradely cannulated with a pulled PE-10 catheter filled with sterile physiological saline (according to von Andrian, 1992). The blood flow in the feeding artery was not obstructed. The location and patency of the cannula was verified by the infusion of 5\% FITC-dextran (MW 150,000; Pharmacia Fine Chemicals AB, Uppsala, Sweden) at the end of each experiment. Fluorescence microscopy with an incident illuminator (Leitz Ploemopak 2.2, tube factor 1.6x, interchangeable filter set $\mathrm{I}_{2}$ ) in combination with a Xenon arc $(150 \mathrm{~W})$ and a high-sensitivity TV camera (Bosch, TYC 9 A, 1 inch SIT tube) showed that the infusion solution had reached all venules under study.

In 7 rabbits no local cannulation was performed. In these rabbits, superfusion of the mesentery with Tyrode's solution was stopped and sterile, pyrogen free saline was superfused. This group is referred to as the superfusion group.

\section{Experimental protocol}

After exteriorization of the mesentery and after cannulation in the infusion group, the tissue was allowed to stabilize for 30 minutes before the experiment started. Then 2 baseline video recordings of 2 minutes each were made.

In the infusion group, the infusion rate was set at a minimal level $(0.1 \mu \mathrm{l} / \mathrm{min})$ during the stabilization period to prevent plugging of the tip of the cannula. At the end of the baseline recordings a total volume of only $4 \mu$ l saline had been infused. Taking into account the place of the tip of the cannula and the size of the cannulated vessel, the infused fluid had not yet reached the circulation under observation. After the baseline recordings, sterile pyrogen free saline was infused at two successive rates of 5 and $10 \mu \mathrm{l} / \mathrm{min}$, for on the average 25 minutes, each followed by a period of 15 minutes during which the infusion rate was again minimal $(0.1 \mu \mathrm{l} / \mathrm{min}) ;$ a negligible amount of saline was delivered to the venules under observation during these periods.

In the superfusion group, superfusion with Tyrode's solution was stopped after the baseline recordings were made. Subsequently, superfusion with sterile pyrogen free physiological saline was started for two periods of 25 and 5 minutes, alternated with superfusion with Tyrode's solution for 15 minutes.

In each experiment 2 to 4 venules were selected, within the diameter range of 18 to $39 \mu \mathrm{m}$. In these microvessels, leukocyte-endothelium interactions were evaluated by determining the level of leukocyte rolling and leukocyte rolling velocity. The level of leukocyte rolling was quantitated off-line by counting in duplicate during 1 to 3 minutes the number of cells that rolled through a predefined venule segment of about $50 \mu \mathrm{m}$ length, and was expressed as the number of rolling cells passing per minute. Leukocyte rolling velocity was determined from the time periods that 10 randomly chosen rolling leukocytes needed to travel a certain distance along the 
vessel wall.

In all selected venules red blood cell (RBC) velocity was determined on-line before and during each experimental step using a prism grating system with the slit covering the whole vessel width (Slaaf et al., 1981). The measured values were divided by a conversion factor of 1.1 (Slaaf et al., 1986; Tangelder et al., 1986) to obtain actual mean RBC velocities. Vascular diameters (range: 18-39 $\mu \mathrm{m}$ ) were measured off-line with an image shearing device (Intaglietta and Tompkins, 1973). Reduced velocity, which is a measure of wall shear rate, was calculated from these parameters (mean RBC velocity/vessel diameter).

\section{Statistics}

Because of their nonsymmetrical distribution, data are presented as medians with interquartile ranges. Because the data suffer from a large degree of variability, they are normalized to baseline values. The normalized data in the figures represent results obtained during the last 5 minutes of each period indicated. Data from both corresponding time periods and corresponding interventions were compared. Paired data groups were compared using the Wilcoxon signed-rank test. Differences between two independent data groups were tested with the Mann-Whitney $U$ test. In all tests, the level of significance was set at 0.05 .

\subsection{Results}

\section{Leukocyte rolling level}

In the infusion group, a median number of 45 leukocytes per minute rolled along the wall of the selected venules after 30 minutes of stabilization $(n=10$; interquartile range: $20-67 / \mathrm{min}$ ). In the superfusion group, the baseline level of leukocyte rolling did not differ from that of the infusion group ( $n=22$; median $33 / \mathrm{min}$, interquartile range: $23-46 / \mathrm{min}$ ). The effect of local administration of saline, either by infusion or superfusion, on the level of leukocyte rolling is presented in Figure 4.1. Superfusion with saline did not induce significant changes in the number of rolling leukocytes. No significant differences existed between both groups. However, infusion of saline (5 $\mu \mathrm{l} / \mathrm{min}$ ) resulted in a significant decrease in the level of leukocyte rolling of approximately $45 \%$ (upper panel; $p \leq 0.05$ vs. baseline); the high infusion rate (10 $\mu \mathrm{l} / \mathrm{min}$ ) did not have an additional effect as compared to the low infusion rate. During the periods in which the infusion rate was minimal $(0.1 \mu \mathrm{l} / \mathrm{min})$, the level of leukocyte rolling did not return to baseline values.

\section{Leukocyte rolling velocity}

Median leukocyte rolling velocity in the baseline situation was $47 \mu \mathrm{m} / \mathrm{s}$ in the infusion group (interquartile range: $38-58 \mu \mathrm{m} / \mathrm{s}$ ) and $51 \mu \mathrm{m} / \mathrm{s}$ in the superfusion group (interquartile range: $34-59 \mu \mathrm{m} / \mathrm{s}$ ) with no difference between the two groups. The effect of local administration of saline, either by infusion or superfusion, on leukocyte rolling velocity is shown in Figure 4.2. Superfusion with saline did not consistently influence leukocyte rolling velocity. Local infusion of saline resulted in a significant 

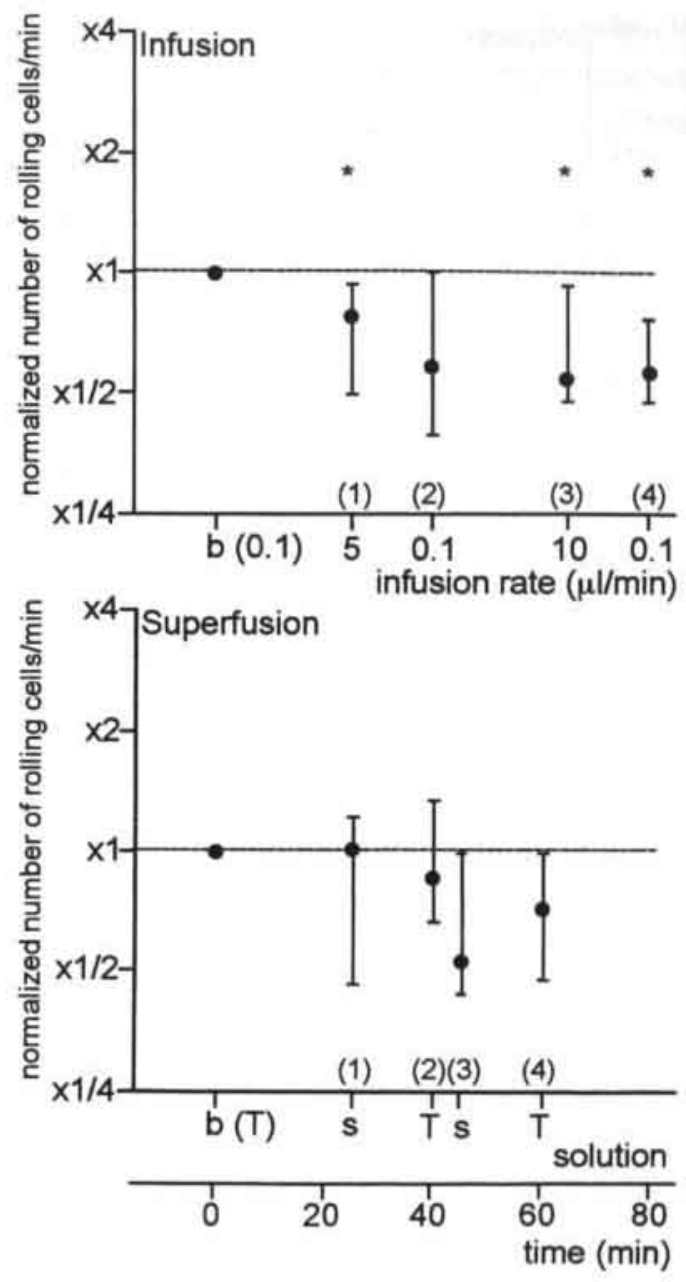

Figure 4.1. Effect of local infusion (top) or superfusion (bottom) of saline on the level of leukocyte rolling in rabbit mesenteric venules. All data are normalized to the baseline values. Medians and interquartile ranges are shown.

* $p \leq 0.05$ as compared to baseline; $b=$ baseline value; $s=s a l i n e ; T=T y r o d e ' s$ solution; $(1)=$ consecutive number of intervention

and persistent increase in leukocyte rolling velocity of about $40 \%(p \leq 0.05$ vs. baseline), even during the periods in which the infusion rate was minimal and a negligible amount of saline was infused. As a result, leukocyte rolling velocity in the infusion group was significantly higher as compared to the velocity in the superfusion group $(p \leq 0.05)$.

\section{Fluid dynamics}

The selected venules in the infusion and superfusion groups had a median diameter 


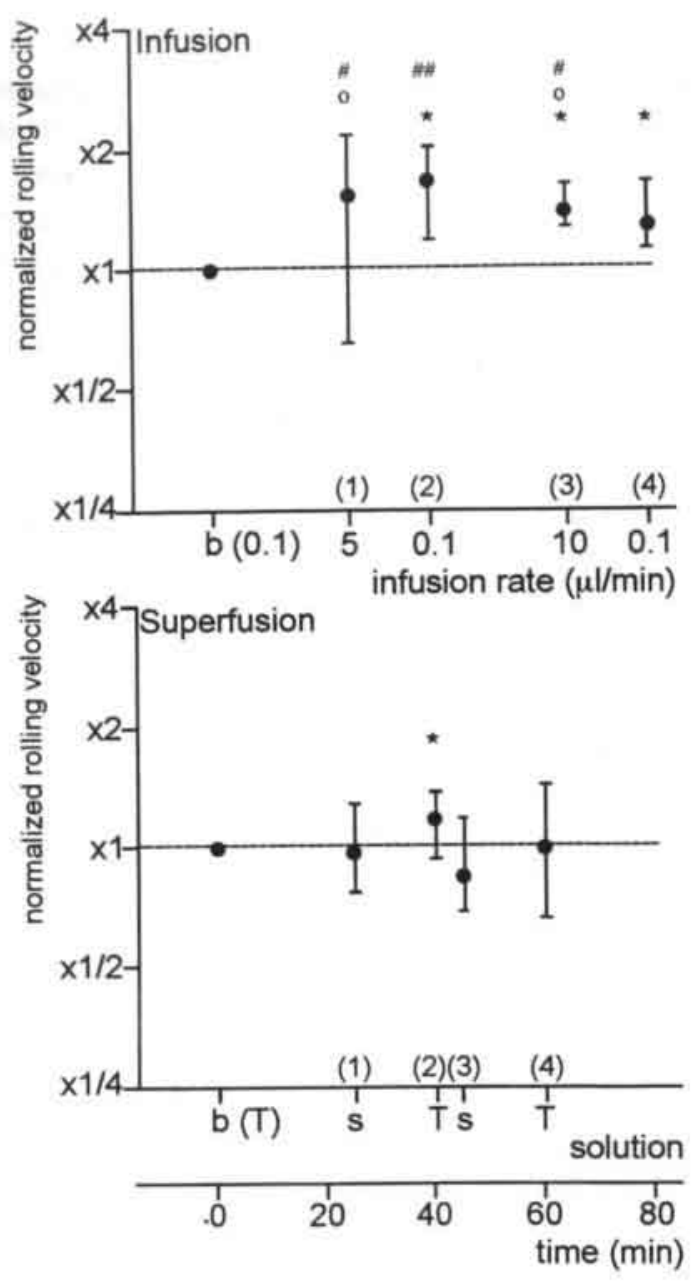

Figure 4.2. Effect of local infusion (top) or superfusion (bottom) of saline on leukocyte rolling velocity in rabbit mesenteric venules. All data are normalized to the baseline values. Medians and interquartile ranges are shown.

* $p \leq 0.05$ as compared to baseline; ${ }^{\circ} \mathrm{p} \leq 0.05$ as compared to corresponding intervention in the superfusion experiments; ${ }^{~} p \leq 0.05$ and ${ }^{\# \prime} p \leq 0.01$ as compared to corresponding time in the superfusion experiments; $b=$ baseline value; $s=s a l i n e ; \quad T=T y r o d e ' s$ solution; (1)=consecutive number of intervention

of 25 and $26 \mu \mathrm{m}$, respectively. In both groups the diameter did not change during the interventions. Baseline median RBC velocity was $1.6 \mathrm{~mm} / \mathrm{s}$ and $1.4 \mathrm{~mm} / \mathrm{s}$ in the infusion and superfusion group, respectively, and reduced velocity, a measure of wall shear rate, was $61 / \mathrm{s}$ and $52 / \mathrm{s}$. These values were not significantly different between both groups. Although significant but small (10-20\%) increases in RBC velocity and reduced velocity were found during the first infusion as well as during superfusion with saline, no differences were found between both groups. 
Baseline mean arterial pressure was $52 \mathrm{mmHg}$ and $63 \mathrm{mmHg}$ in the infusion and the superfusion group, respectively, and baseline heart rate was 120 and 144 beats per minute. In both groups, these parameters did not change in the course of the experiments.

\subsection{Discussion}

Saline is commonly used as the vehicle for water-soluble agents with presumed effects on the microcirculation. Such agents are often administered locally through infusion via a small feeding artery or by topical superfusion. In this kind of studies, it is a standard procedure to perform control experiments with saline alone, and in general no major effects are to be expected. In the present study, however, local infusion of saline appeared to have a clear influence on leukocyte-endothelium interactions, despite a large variability of the data: leukocyte rolling velocity increased significantly and the number of rolling leukocytes decreased. These effects were not found after topical application of saline. This is an unexpected and undesirable finding, that should be kept in mind when planning experiments in which effects of locally administered agents are to be studied.

Thirty minutes after manipulation of the exteriorized mesentery leukocyteendothelium interactions in the infusion and superfusion group were similar. Differences in leukocyte-endothelium interactions between the infusion and the superfusion group were found only after the infusion rate was increased to $5 \mu / \mathrm{min}$. a rate at which saline was, for the first time, actually delivered to the circulation, and, henceforth, to the venules under observation. This indicates that the mere presence of a local cannula in a distal small mesenteric artery, for instance for blood pressure measurements (Fenger-Gron et al., 1995), does not influence leukocyte-endothelium interactions in downstream venules. However, when the cannula is used for infusion of fluids one should be aware that leukocyte-endothelium interactions may be affected. Furthermore, we observed that changes in the level of leukocyte rolling and in rolling velocity persisted, between the two infusion steps of 5 and $10 \mu / \mathrm{min}$, when the infusion rate was minimal; a situation in which leukocytes in the venular blood stream were only very minimally exposed to the infused fluid. This suggests that the findings of the present study are mainly due to a long lasting effect on the venular endothelium.

Since blood flow in the arteries feeding the mesenteric segments under observation was in the order of $0.30-0.35 \mathrm{ml} / \mathrm{min}$ (unpublished data), the infusion rates used in the present study ( 5 and $10 \mu / / \mathrm{min}$ ) will have caused an increase in blood flow and a reduction in hematocrit in the downstream blood vessels of only $2-3 \%$. This explains why local infusion of saline did not induce any changes in venular hemodynamics, such as diameter, RBC velocity or reduced velocity as compared to superfusion of saline. Von Andrian and coworkers (von Andrian et al., 1992) who used the same model, infused on the average $560 \mu$ phosphate-buffered saline during 1 minute and found that venular hemodynamics transiently increased (with on the average 13\%) during injection. In experiments performed by Ley and 
coworkers (Ley et al., 1991a) in which substances were infused into mesenteric venules themselves via glass micropipettes, wall shear rate during microinfusion was increased to 2 times preinfusion values; the changes observed in leukocyte rolling had to be corrected for local dilution of the blood and the accompanying decrease in leukocyte concentration. In our experiments no hemodynamic changes occurrec; therefore, the effects of saline cannot be explained by hemodynamic factors, while no correction for leukocyte concentration is required.

In summary, this study indicates that saline, locally infused into a smal] mesenteric artery, reduces leukocyte-endothelium interactions in downstrean venules, whereas superfused saline has no effect. Such an undesired effect of an infused vehicle like saline may conceal effects of water-soluble agents, if any, on microvascular parameters. Since superfusion of saline did not influence leukocyte: endothelium interactions, topical administration of agents should be the method of choice. When local infusion techniques are to be used, a vehicle control must be performed, preferably within the same experiment. 



\section{ARE MAST CELLS INVOLVED IN REGULATION OF LEUKOCYTE ROLLING IN RABBIT MESENTERIC VENULES?}

Based on: S.C. Tromp, D.W. Slaaf, E. van Breda, G.J. Tangelder, R.S. Reneman, E.V. Rouwet, and M.G.A. oude Egbrink. Are mast cells involved in regulation of leukocyte rolling in mesenteric venules of anesthetized rabbits? In: Proceedings of the sixth world congress for microcirculation. Eds: K.Messmer and W.M. Kuebler. Monduzzi Editore, Bologna, 1996, pp 649-653

\section{Abstract}

Mast cell degranulation and the consequent release of histamine has been shown to induce leukocyte rolling in mesenteric venules of Sprague-Dawley rats. Since it is unknown whether this also applies to rabbits, we investigated in this species the effects of mast cell stabilization, mast cell degranulation, and histamine receptor blockade on leukocyte rolling in mesenteric venules: no effects were found. In an additional histological study, no mast cells appeared to be present in the mesentery of rabbits. It is concluded that mast cells are not involved in induction and regulation of leukocyte rolling in mesenteric venules of the rabbit. 


\subsection{Introduction}

Rolling of leukocytes along the endothelium of postcapillary venules appears to be a necessary first step, preceding adhesion and diapedesis, in the reaction of leukocytes to inflammatory stimuli (von Andrian et al., 1991; Springer, 1994). Intravital videomicroscopic studies on leukocyte rolling are often performed in the mesentery (Atherton and Born, 1972; House and Lipowsky, 1987; Tangelder and Arfors, 1991; oude Egbrink et al., 1992; Beuk et al., 1996). After surgical exposure of the mesentery in such studies, leukocyte rolling can always be observed.

Recently, it has been shown that in rats degranulation of mast cells due to exteriorization of the mesentery is responsible for the induction of leukocyte rolling in mesenteric venules (Kubes and Kanwar, 1994; Ley, 1994). Pretreatment of rats with the connective tissue mast cell stabilizer cromoglycate resulted in a profound reduction in baseline leukocyte rolling; subsequent histamine superfusion increased leukocyte rolling via a P-selectin-dependent mechanism (Kubes and Kanwar, 1994).

It is unknown whether mast cell degranulation and consequent histamine release are also responsible for the induction of leukocyte rolling in mesenteric venules in rabbits. Therefore, we investigated in this species the effect on leukocyte rolling in mesenteric venules of (1) mast cell stabilization prior to mesentery preparation and (2) subsequent mast cell degranulation with or without histamine receptor blockade. Furthermore, histological whole mount preparations of the mesentery were stained to study the presence and degree of degranulation of mast cells.

\subsection{Materials and methods}

Using intravital video microscopy, venules (diameters: 19 to $37 \mu \mathrm{m}$ ) were studied in the mesentery of 12 anesthetized New Zealand white rabbits $(40 \mathrm{mg} / \mathrm{kg} / \mathrm{hr}$ ketamine and 5 $\mathrm{mg} / \mathrm{kg} / \mathrm{hr}$ xylazine i.v.). The mast cell stabilizer cromoglycate $(20 \mathrm{mg} / \mathrm{kg}$ i.v.) was administered to all rabbits prior to surgical preparation of the mesentery. After exteriorization, the mesentery was allowed to stabilize for 30 minutes. Subsequently, two recordings of two minutes each were made to determine baseline leukocyte rolling. After these recordings, the mast cell degranulator compound $48 / 80$ was added to the superfusate of 9 rabbits in subsequent concentrations of 3 and $10 \mu \mathrm{g} / \mathrm{ml}$. Each dose was given for 5 minutes, followed by 25 minutes washout. The level of leukocyte rolling was determined in the last 15 minutes of each washout period. In addition, 6 of these rabbits received chlorpheniramine as well as cimetidine $\left(\mathrm{H}_{1}-\right.$ and $\mathrm{H}_{2}$-receptor antagonists, respectively), before mesenteric preparation (both $5 \mathrm{mg} / \mathrm{kg}$ i.v.), and also in the superfusate (both $10^{-5} \mathrm{M}$ ) during the whole experiment. Three rabbits, of which the mesentery was not superfused with compound $48 / 80$, served as control.

The level of leukocyte rolling was determined by counting the number of rolling leukocytes passing through a segment of the vessel during 2 minutes. Leukocyte rolling velocity was determined by measuring the time needed for 10 randomly chosen rolling leukocytes to travel a distance of 50 to $100 \mu \mathrm{m}$. Mean blood cell velocity, vascular diameter, and mean arterial pressure were also measured (see for details chapter 8 ). 
Reduced velocity, i.e., a measure for wall shear rate, was calculated (mean blood cell velocity divided by vascular diameter). Systemic leukocyte counts were determined before administration of cromoglycate and at the end of each experiment.

After each experiment, whole mount preparations of the mesentery were made and stained with Toluidine Blue $\mathrm{O}$ in concentrations ranging from 0.05 to $1 \%$ with $\mathrm{pH}$ ranging from 2.1 to 6.8 . Furthermore, several other techniques for fixation of the preparation (formaldehyde $4-10 \%$, ethanol $70 \%$, aceton, glutaaraldehyde $1 \%$ ) and staining of mast cells (Safranine red, Bismarck brown) in various species and tissues were applied (Tas and Geenen, 1975; Mendonca et al., 1986; Raud et al., 1989; Fiebig et al., 1991; Kubes, 1993; Ley, 1994).

Paired data groups were compared with the Wilcoxon signed-rank test. Differences between two or more independent data groups were tested with the Mann-Whitney $U$ test and the Kruskal-Wallis test, respectively.

\subsection{Results and discussion}

Baseline levels of leukocyte rolling following administration of cromoglycate alone (median: $46 / \mathrm{min}$, interquartile range: $30-79 / \mathrm{min}$ ) or in combination with histamine receptor antagonists $(44 / \mathrm{min}, 30-64 / \mathrm{min})$ were within ranges that are normally found in untreated rabbits. In Figure 5.1 these values are compared to baseline levels of leukocyte rolling in a group of rabbits in which no cromoglycate was administered (see chapter 7 , infusion control group). No significant differences existed between the three groups. Therefore, mast cell stabilization before exteriorization of the mesentery does not prevent the induction of leukocyte rolling in rabbits.

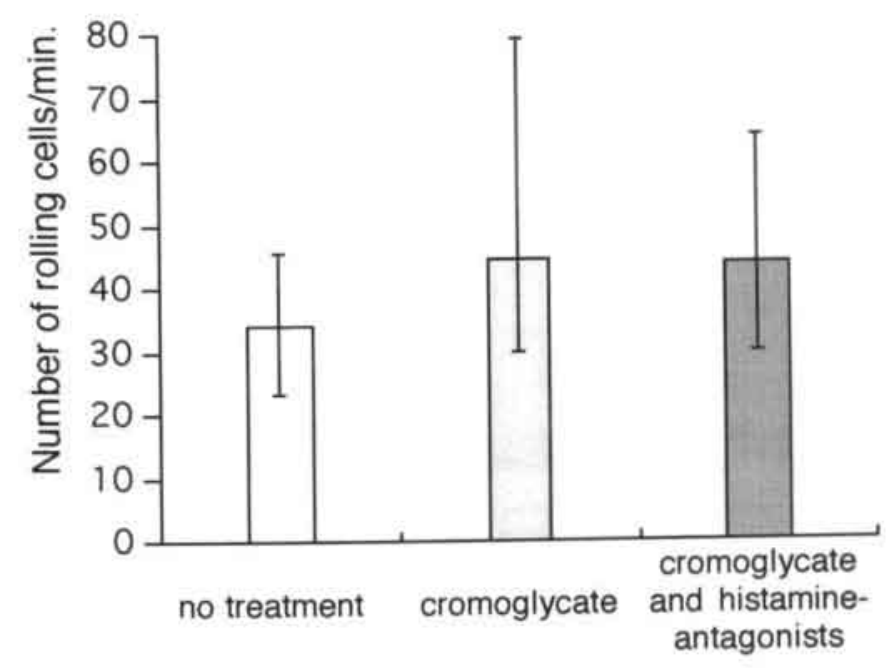

Figure 5.1. Baseline levels of leukocyte rolling in mesenteric venules in control rabbits (see chapter 7 ), rabbits pretreated with cromoglycate, or with cromoglycate and histamine receptor antagonists. Medians and interquartile ranges are presented. 
The effects of time (control group) and compound $48 / 80$ on the level of leukocyte rolling in mesenteric venules of mast cell-stabilized rabbits is shown in Figure 5.2A. In the control group, the level of leukocyte rolling significantly decreased in time to approximately $50 \%$. A similar decrease over the same period of time has been observed previously in untreated rabbits (unpublished data). Administration of compound 48/80 to the superfusate did not alter this decrease in level of leukocyte rolling. Figure 5.2B shows the effects of time and compound $48 / 80$ on leukocyte rolling velocity in mesenteric venules of rabbits treated with cromoglycate. No changes in leukocyte rolling velocity were found in time in the control group. In the groups receiving compound $48 / 80$, leukocyte rolling velocity showed small but significant increases. However, no differences were found between the three groups.

The median baseline diameters of the venules of the control group and of the groups without and with histamine receptor blockade, were 25,25 , and $27 \mu \mathrm{m}$, respectively. In neither of these groups the diameter changed during the experiments. Median blood cell

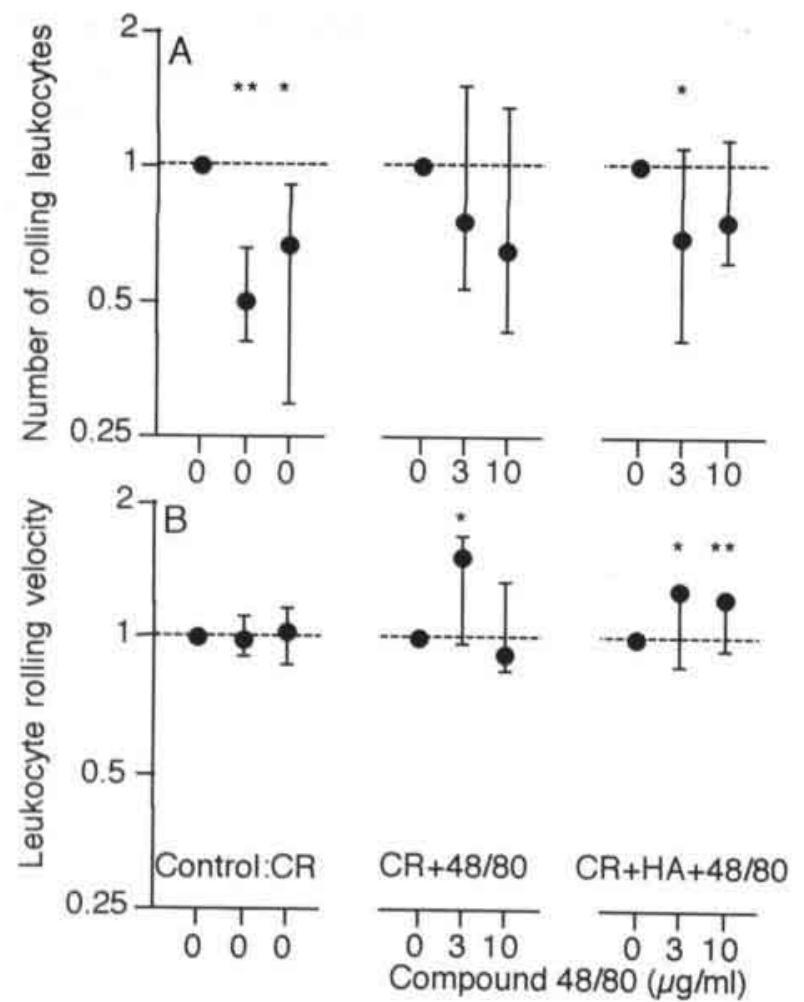

Figure 5.2. Effect of time and compound $48 / 80$ on (A) level of leukocyte rolling and (B) leukocyte rolling velocity in mesenteric venules of mast cell-stabilized rabbits. Medians and interquartile ranges (bars) are shown. Data are normalized to baseline values.

* $p<0.05$ and * $p<0.01$ as compared to baseline values $(0 \mu \mathrm{g} / \mathrm{ml}$ compound $48 / 80)$; $\mathrm{CR}=$ pretreated with cromoglycate; $\mathrm{HA}=$ pretreated with histamine receptor antagonists 
velocities were $1.3,1.4$, and $1.7 \mathrm{~mm} / \mathrm{s}$ and median reduced velocities were 59,61 , and $63 / \mathrm{s}$, respectively; no changes occurred during the experiments and no differences between the groups were found. Mean arterial pressures were within normal ranges in all rabbits and did not change in the course of the experiments. Basic systemic leukocyte counts were normal (median: $6.4 \times 10^{6} / \mathrm{ml}$ blood) and no changes occurred during the experiments. These results lead to the conclusion that neither hemodynamic parameters nor systemic leukocyte counts have influenced leukocyte rolling in the rabbits under investigation.

The results of the present study indicate that mast cell stabilization before exteriorization of the mesentery and subsequent administration of the mast cell degranulator compound $48 / 80$ with or without histamine receptor blockade does not influence leukocyte rolling in rabbit mesenteric venules. Therefore, in contrast to Sprague-Dawley rats, mast cells and their contents do not seem to be involved in the induction of leukocyte rolling in mesenteric venules in rabbits. This finding is not surprising in view of our histological data. Although several techniques for preparation and staining of the tissue were used, no mast cells could be found in the rabbit mesentery. Mast cells can be found in other tissues of the rabbit with the use of similar techniques (Abelson et al., 1983; Ennis et al., 1987; Chole and Kodama, 1989).

In conclusion, degranulation of mast cells due to surgical exteriorization of the mesentery cannot be held responsible for the rapid induction of leukocyte rolling in mesenteric venules of rabbits. It has been shown in this species that rolling is absent in mesenteric venules before exteriorization of the tissue (Fiebig et al., 1991). Therefore, another, presently unknown mechanism is responsible for the induction of leukocyte rolling in the mesentery of rabbits. The present study also indicates that the induction of leukocyte rolling in tissues like the mesentery is species-dependent. 



\section{THE ROLE OF MAST CELLS AND HISTAMINE IN LEUKOCYTE- ENDOTHELIUM INTERACTIONS IN FOUR RAT STRAINS}

Published as: S.C. Tromp, G.J. Tangelder, D.W. Slaaf, R.S. Reneman, J.S van Velzen, W. Engels, E. van Breda, and M.G.A. oude Egbrink. The role of mast cels and histamine in leukocyte-endothelium interactions in four rat strains. Pflügers Archives - European Journal of Physiology (1998) 436:255-261

\section{Abstract}

The objective of the present study was to determine the role of mast cells and histamine in leukocyte-endothelium interactions in mesenteric venules of four rat strains: Brown Norway, Lewis, Sprague-Dawley, and Wistar rats. Intravital microscopy showed that the mast cell stabilizer cromoglycate $(5 \mathrm{mg} / \mathrm{kg}$ i.v. just before exteriorization of the mesentery) did not affect the baseline level and velocity of leukocyte rolling in any of the four rat strains. This finding is in agreement with the observation that cromoglycate pretreatment only slightly influenced mast cell degranulation in all rat strains except the Brown Norway rats. After mast cell stabilization, only in Sprague-Dawley rats topical administration of histamine $\left(10^{-4} \mathrm{M}\right)$ resulted in a significant increase in the level of leukocyte rolling and a decrease in the rolling velocity as compared to time control. Histamine induced leukocyte adhesion only in Brown Norway rats. In conclusion, the hypothesis presented in other studies that degranulation of mast cells, and more specifically the release of histamine, is of major importance for the induction of leukocyte-endothelium interactions in rat mesenteric venules is not generally applicable; the present study clearly shows strain-dependency. 


\subsection{Introduction}

Rolling along the endothelium of postcapillary venules appears to be the first step in the response of leukocytes to inflammatory stimuli. This initial weak interaction of leukocytes with the endothelium is a prerequisite for subsequent firm adhesion, diapedesis, and migration into the tissue (von Andrian et al., 1992; Springer, 1994). There are indications that inflammatory mediators released by mast cells, such as histamine, may stimulate certain steps in the cascade from free flowing leukocytes to the accumulation of these cells in tissues. The involvement of mast cells in this cascade has, for example, been demonstrated in IgE-dependent inflammatory reactions (Raud et al., 1989; Wershil et al., 1991) and in ischemia-reperfusion injury (Kanwar and Kubes, 1994). In Sprague-Dawley rats degranulation of mast cells, due to exteriorization of the mesentery, has been suggested to be responsible for induction of the initial contact of leukocytes with the vessel wall, i.e., leukocyte rolling (Kubes and Kanwar, 1994). Pretreatment of these rats with the connective tissue mast cell stabilizer sodium cromoglycate resulted in a profound reduction in baseline leukocyte rolling in mesenteric venules, while subsequent histamine superfusion increased leukocyte rolling via a P-selectin-dependent mechanism (Kubes and Kanwar, 1994). Experiments performed in vitro have demonstrated that histamine can directly activate endothelial cells to redistribute P-selectin to the cell surface (Hattori et al., 1989), inducing leukocyte rolling on endothelial monolayers (Jones et al., 1993).

Recent experiments in our laboratory, however, indicated that in rabbits mast cells play little or no role in the induction of leukocyte rolling in mesenteric venules (Tromp et al., 1996). Unlike in rat mesentery, histological evaluation of whole mount preparations of rabbit mesentery showed hardly any mast cells. Moreover, in rabbits mast cell stabilization as well as anti-histamine treatment did not affect leukocyte rolling (Tromp et al., 1996). Additional preliminary studies in our laboratory indicated that, beside species differences, there may also be differences in this respect between various strains within one species, because we could not find any effect of mast cell stabilization on baseline leukocyte rolling in Lewis rats either.

Therefore, the objective of the present study was to determine in vivo the role of mast cells and of histamine in leukocyte-endothelium interactions in mesenteric venules of four rat strains, that is Brown Norway, Lewis, Sprague-Dawley, and Wistar rats. Two specific issues were addressed: (a) to what extent does the mast cell stabilizer sodium cromoglycate affect degranulation of mast cells and leukocyte rolling; and (b) what is the effect of exogenously administered histamine on leukocyte rolling and adhesion after mast cell stabilization.

\subsection{Materials and methods}

\section{Animals and intravital videomicroscopy}

The experiments were approved by the local ethical committee on the use of laboratory animals. They were performed on 14 Brown Norway rats (BN), 21 Lewis 
rats (LE), 19 Sprague-Dawley rats (SD), and 20 Wistar rats (WI). BN (official name $\mathrm{BN} / \mathrm{m}$ ) and LE (Lewis/m) were obtained from the animal facilities of Maastricht University, the Netherlands, while SD and WI were purchased from Charles River Laboratories, Germany. The male rats were 10-12 weeks of age and body weights ranged from 200 to $280 \mathrm{~g}$ in $\mathrm{BN}$, from 200 to $370 \mathrm{~g}$ in LE, from 210 to $370 \mathrm{~g}$ in SD, and from 230 to $330 \mathrm{~g}$ in WI.

Anesthesia was induced with an intraperitoneal injection of sodium pentobarbital ( $6.6 \mathrm{mg} / 100 \mathrm{~g}$ body weight), given under a short $(<20 \mathrm{~s}) \mathrm{CO}_{2}$ sedation $\left(40 \% \mathrm{CO}_{2}\right.$ in air), and was maintained by continuous intravenous infusion of sodium pentobarbital $(24 \mathrm{mg} / \mathrm{kg} / \mathrm{h})$ through a catheter $(\mathrm{PE}-10)$ in the right femoral vein. This anesthetic procedure does not influence leukocyte rolling (Janssen et al., 1997). Arterial blood pressure was measured continuously through a catheter (PE-10) in the femoral artery (Uniflow external pressure transducer, Baxter, Santa Ana, CA, U.S.A.). To keep the arterial catheter patent it was continuously perfused with physiological saline $(1 \mathrm{ml} / \mathrm{h}$; Uniflow system). Heart rate was assessed from the instantaneous pressure signal. Mean arterial pressure and heart rate were continuously recorded on a computer harddisk using a data acquisition system. Throughout the experiments body temperature was kept at $37^{\circ} \mathrm{C}$ by means of an infrared heating lamp controlled by a thermo-analyzer system connected to a rectal probe, and by placing the rats on a heating pad $\left(37^{\circ} \mathrm{C}\right)$.

Through a right flank incision a segment of the distal ileum was exteriorized and the mesentery was carefully spread over a siliconized glass plate mounted in an electrically heated microscope table $\left(37^{\circ} \mathrm{C}\right)$. The preparation was continuously superfused with buffered Tyrode's solution $\left(37^{\circ} \mathrm{C} ; \mathrm{pH} 7.35-7.40\right)$ bubbled with a mixture of $\mathrm{N}_{2}(95 \%)$ and $\mathrm{CO}_{2}(5 \%)$. The bowels were kept moist with overlying wet gauze and covered with clear plastic wrap to minimize tissue dehydration. On the average 3 mesenteric venules per rat were visualized using a Leitz intravital microscope, adapted for telescopic imaging (Slaaf et al., 1982) and equipped with a water-immersion objective (SW25, numerical aperture 0.60 ). Transillumination was performed with a tungsten lamp. Images were recorded on videotape (Sony, Betamax) through a TV camera (Bosch, Ultricon 4532, 1 inch). Final optical magnification at the front plane of the camera was $\times 52$.

Venular diameters (range: 17-39 $\mu \mathrm{m}$ ) were measured off-line with an image shearing device (Intaglietta and Tompkins, 1973), while red blood cell (RBC) velocity was determined on-line using a prism grating system with the slit covering the whole vessel width (Slaaf et al., 1981). To obtain actual mean RBC velocities, the measured velocity values were divided by a conversion factor of 1.1 (Slaaf et al., 1986; Tangelder et al., 1986). Reduced velocity (mean RBC velocity/vessel diameter), which is a measure of wall shear rate, was calculated from these parameters.

Leukocytes were considered as rolling if by eye they could be seen moving along the vessel wall, either at a rather constant velocity or saltating at a significantly lower rate than the blood was flowing. To assure that all rolling leukocytes could be distinguished, the midplane of a venule was kept in focus in all experiments (oude 
Egbrink et al., 1992). To describe leukocyte rolling the level of leukocyte rolling as well as the rolling velocity was determined. Leukocyte rolling level was quantitated off-line by counting in duplicate the number of cells that rolled along a predefined segment of the venule during a period of 100 to 120 seconds. The level of leukocyte rolling was expressed as the number of rolling cells passing per minute. The velocity of the rolling leukocytes was determined off-line by measuring the time needed for 10 randomly chosen rolling leukocytes to travel a certain distance along the vessel wall. Leukocytes were considered to be adherent when they remained stationary for at least $30 \mathrm{~s}$. The number of adherent leukocytes was determined in a $100 \mu \mathrm{m}$ segment of the venule. The mean number present at any instant was assessed over a period of 2 minutes.

\section{Experimental protocol}

To evaluate the extent to which mast cell stabilization influences the induction of leukocyte rolling by the surgery and tissue exteriorization, all rats from each strain were assigned at random to one of two groups. One of these groups was pretreated with the connective tissue mast cell stabilizer cromoglycate $(\mathrm{Cr}+$ group) according to the protocol used by Kubes and colleagues (Kubes and Kanwar, 1994), while the other group was not ( $\mathrm{Cr}$ - group). Cromoglycate (cromolyn sodium salt, Sigma Chemical, St. Louis, MO, U.S.A.) was dissolved in sterile saline and administered (5 $\mathrm{mg} / \mathrm{kg}$ i.v.) to the rats of the $\mathrm{Cr}+$ group 2 minutes before surgical exteriorization of the mesentery. After exteriorization the tissue was allowed to stabilize for 30 minutes in both groups. Subsequently RBC velocity was measured and video recordings were made to assess baseline leukocyte rolling.

To evaluate the effect of exogenously administered histamine on leukocyte rolling and adhesion, the cromoglycate treated animals were divided into two subgroups. Following baseline measurements, in one of these two subgroups histamine dihydrochloride (Sigma Chemical, St. Louis, MO, U.S.A.) was added to the Tyrode's solution in subsequent concentrations of $10^{-5} \mathrm{M}$ and $10^{-4} \mathrm{M}$, for 25 minutes each $(\mathrm{Cr}+\mathrm{Hi}+$ group). In the other subgroup no histamine was added to the superfusate $\left(\mathrm{Cr}+\mathrm{Hi}\right.$ - group). This group served as time control. Data obtained with $10^{-5} \mathrm{M}$ and $10^{-4} \mathrm{M}$ histamine in the $\mathrm{Cr}+\mathrm{Hi}+$ group are compared to those obtained at $\mathrm{t}=25$ minutes and $t=50$ minutes following baseline, respectively, in the $\mathrm{Cr}+\mathrm{Hi}$ - group. The effect of histamine was also investigated in animals that were not pretreated with cromoglycate following the same protocol as used in the cromoglycate treated rats. All fluids were superfused at a rate of $150-250 \mathrm{ml} / \mathrm{h}$. Temperature of the superfusion fluid was kept at $37^{\circ} \mathrm{C}$. Throughout the experiments RBC velocities were measured and video recordings were made for off-line analysis.

\section{Systemic leukocyte counts}

Systemic leukocyte counts were performed at the beginning of the stabilization period and at the end of each experiment ( $t=75$ minutes following baseline). To this purpose, $20 \mu \mathrm{l}$ blood was collected through the arterial catheter and administered to Türks solution ( $0.2 \mathrm{mg}$ gentian violet in $1 \mathrm{ml}$ glacial acetic acid, $6.25 \% \mathrm{vol} / \mathrm{vol}$ ) in a 
1:10 dilution; leukocytes were counted and differentiated as polymorphonuclear (PMN) or monomorphonuclear (MMN) in a counting chamber (Clay Adams, Parsippany, N.J., U.S.A.).

\section{Histology}

At the end of each experiment (duration in all cases approximately 2 hours) the mesenteric segment of observation was carefully excised and dried at room temperature on a glass slide as whole mount. Tissues were stained with Toluidine Blue O (Sigma Chemical, St. Louis, MO, U.S.A.; $0.1 \%$ in $50 \mathrm{mM}$ citric acid, pH 2.2) for 1 minute, briefly rinsed in water, dehydrated through graded alcohols, cleared in xylene, and embedded in Entellan (Merck, Darmstadt, Germany). Mast cells were counted in microscopic fields of approximately $1.6 \mathrm{~mm}^{2}$. Mast cell density was determined by averaging the numbers of 2 to 9 microscopic fields per whole mount and expressed per $\mathrm{mm}^{2}$ tissue area. The perivenular number of mast cells was determined by counting over a vessel length of $200 \mu \mathrm{m}$ the mast cells located within $50 \mu \mathrm{m}$ distance from the venules that had been observed in vivo. Perivenular mast cell density was also expressed per $\mathrm{mm}^{2}$ tissue area. All mast cells were classified morphologically (Wershil et al., 1991) as not degranulated, moderately degranulated (10-50\% of the granules expelled from the cell), or extensively degranulated (50$100 \%$ of the granules expelled).

\section{Statistics}

Because of their nonsymmetrical distribution, data are presented as medians with interquartile ranges (i.e., the spread from 25 th to 75 th percentile). The data shown in the figures 2 to 4 represent results obtained during the last 5-10 minutes of each experimental period indicated. To enable comparisons between groups, the relative change in the level of leukocyte rolling was computed: data are presented normalized to (i.e., divided by) their individual baseline values. Effects of histamine, if any, are compared to the effects of time. Paired data groups were compared using the Wilcoxon signed-rank test. Differences between two independent data groups were tested with the Mann-Whitney $\mathrm{U}$ test; to compare more than two independent data groups the Kruskal-Wallis test was used. In all tests, the level of significance was set at 0.05 .

\subsection{Results}

\section{Systemic parameters}

Baseline mean arterial pressure was lowest in Brown Norway (BN; median 107 $\mathrm{mmHg} ; \mathrm{p} \leq 0.01$ ) and higher in Lewis (LE; $113 \mathrm{mmHg}$ ), Sprague-Dawley (SD; 110 $\mathrm{mmHg}$ ), and Wistar rats ( $\mathrm{Wl} ; 120 \mathrm{mmHg})$. Median baseline heart rate was 359,380 , 392 , and 384 beats per minute in BN, LE, SD, and WI, respectively, being highest in SD $(p \leq 0.01)$. Superfusion with $10^{-5} \mathrm{M}$ histamine had no effect on mean arterial pressure, whereas a concentration of $10^{-4} \mathrm{M}$ histamine caused a decrease by $13 \%$ in $\mathrm{LE}, 12 \%$ in SD, and $7 \%$ in $\mathrm{WI}$ and had no effect in $\mathrm{BN}$. Heart rate remained constant 
during the experiments in all groups.

In all groups peristalsis of the small bowel, with an increasing intensity towards the end of the experiment, was observed in some rats. No relation was found between bowel movements and leukocyte rolling or adhesion.

Median systemic number of leukocytes at the beginning of the experiments were within the normal range (Ringler and Dabich, 1979): $10.0 \times 10^{6} / \mathrm{ml}$ blood in BN, 8.8$\times 10^{6} / \mathrm{ml}$ in LE, $9.6 \times 10^{6} / \mathrm{ml}$ in SD, and $10.6 \times 10^{6} / \mathrm{ml}$ in WI rats. In the course of the experiments the systemic number of leukocytes did not change and no differences between the strains were found. The percentage of polymorphonuclear cells (PMNs) increased substantially during the experiments in all four rat strains: from a median of $5 \%$ (before exteriorization of the mesentery) to $51 \%$ (at $t=75$ minutes following baseline) in BN, from $10 \%$ to $55 \%$ in LE, from $15 \%$ to $48 \%$ in SD, and from $10 \%$ to $55 \%$ in WI. The increases were similar in all experimental groups.

\section{Effect of cromoglycate on degranulation of mast cells}

The mesenteric mast cell density differed significantly between the rat strains, being lower in BN (median 34/ $\mathrm{mm}^{2}$ ) and LE $\left(42 / \mathrm{mm}^{2}\right)$ and higher in SD $\left(60 / \mathrm{mm}^{2}\right)$ and WI $\left(57 / \mathrm{mm}^{2}\right)$. Figure 6.1 shows the effect of the mast cell stabilizer cromoglycate on mast cell degranulation in the four rat strains. Without cromoglycate $10 \%$ (WI) to $47 \%(\mathrm{BN})$ of the mast cells degranulated moderately or extensively upon exteriorization; the difference between all strains being significant $(p \leq 0.005)$. Cromoglycate pretreatment did not stabilize all mast cells: the percentage of degranulated mast cells in pretreated animals varied from $9 \%(\mathrm{WI})$ to $27 \%(\mathrm{BN})$; the difference between all strains was still highly significant. The effect of cromoglycate

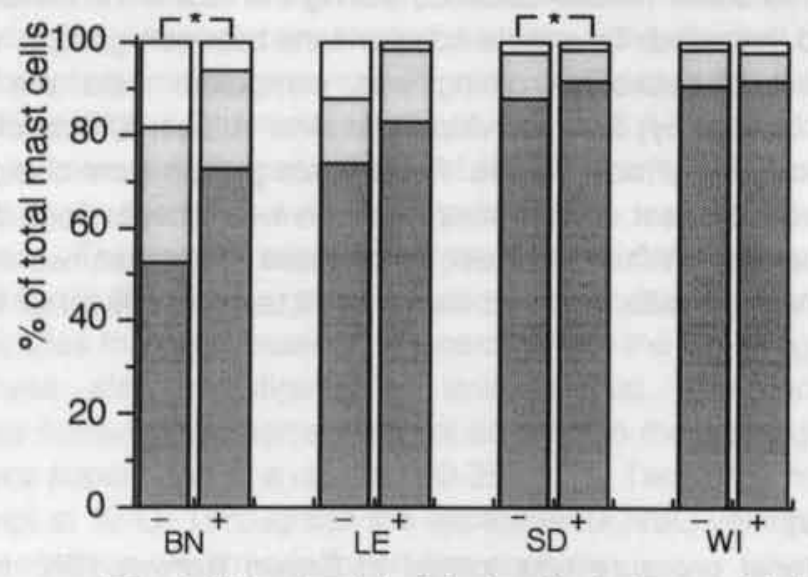

not, $\square$ moderately, $\square$ extenstively degranulated

Figure 6.1. Effect of cromoglycate treatment on the degranulation of mesenteric mast cells in four rat strains. Y-axis shows the percentage of cells with the indicated level of degranulation.

$-=$ not pretreated with cromoglycate $(\mathrm{Cr} \text { - group })_{i}+=$ pretreated $(\mathrm{Cr}+$ group $)$

* ps0.05 as compared to the $\mathrm{Cr}$ - group; indicates significant differences between percentages of mast cells that are (moderately and extensively) degranulated 
was most profound in $\mathrm{BN}$, where the percentage of (moderately and extensively) degranulated cells was significantly decreased. In the other rat strains the effect of cromoglycate was relatively small, though significant in SD ( $\leq \leq 0.05)$; cromoglycate reduced the percentage of extensively degranulated mast cells significantly in BN $(p=0.02)$ and almost significantly in LE rats $(p=0.06)$.

Perivenular mast cell density differed between the rat strains $(p \leq 0.01)$, being lowest in LE (median $100 / \mathrm{mm}^{2}$ tissue area) and higher in BN $\left(150 / \mathrm{mm}^{2}\right), S D$ $\left(200 / \mathrm{mm}^{2}\right)$ and WI $\left(200 / \mathrm{mm}^{2}\right)$. No relationship could be found between vessel diameter and perivenular mast cells density. Following exteriorization, in untreated rats $(\mathrm{Cr}$ - group) the perivenular mast cells did not degranulate in SD and $\mathrm{WI}$, while $33 \%$ and $11 \%$ of the cells degranulated in BN and LE, respectively (all medians). After treatment with cromoglycate $(\mathrm{Cr}+$ group), no perivenular mast cell degranulation could be found in any of the strains.

Effect of cromoglycate on the induction of leukocyte rolling

Mast cell stabilization by cromoglycate pretreatment did not significantly affect the level of leukocyte rolling nor the leukocyte rolling velocity in any of the rat strains, as shown in Figure 6.2A and B, respectively.
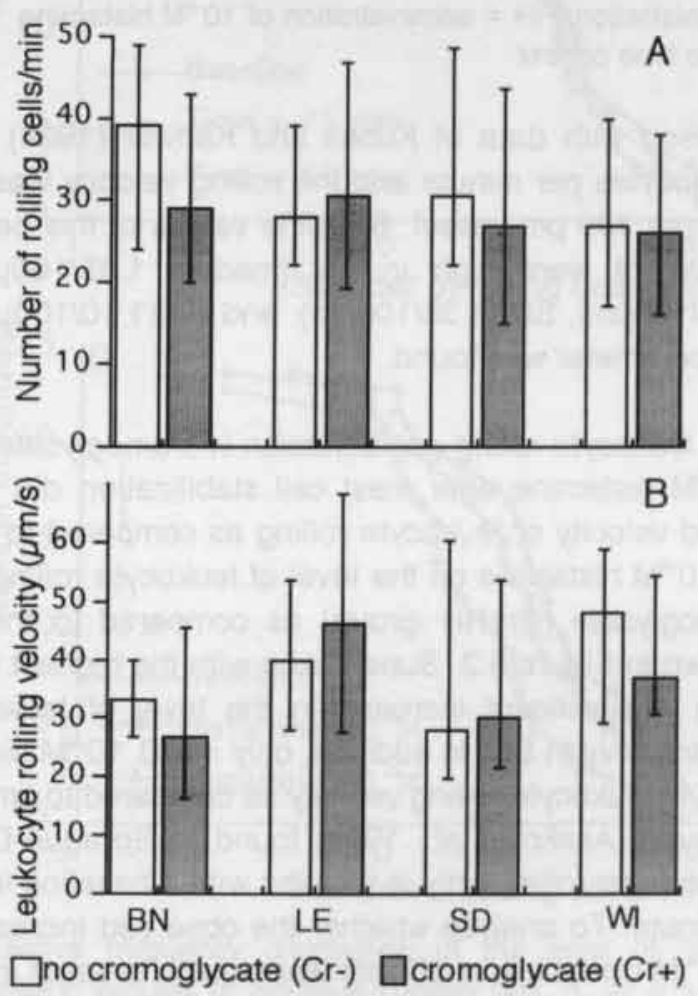

Figure 6.2. Effect of treatment with cromoglycate before surgical exteriorization of the mesentery on the baseline level of leukocyte rolling (A) and on leukocyte rolling velocity (B) in four different strains of rats. Medians and interquartile ranges are presented. 


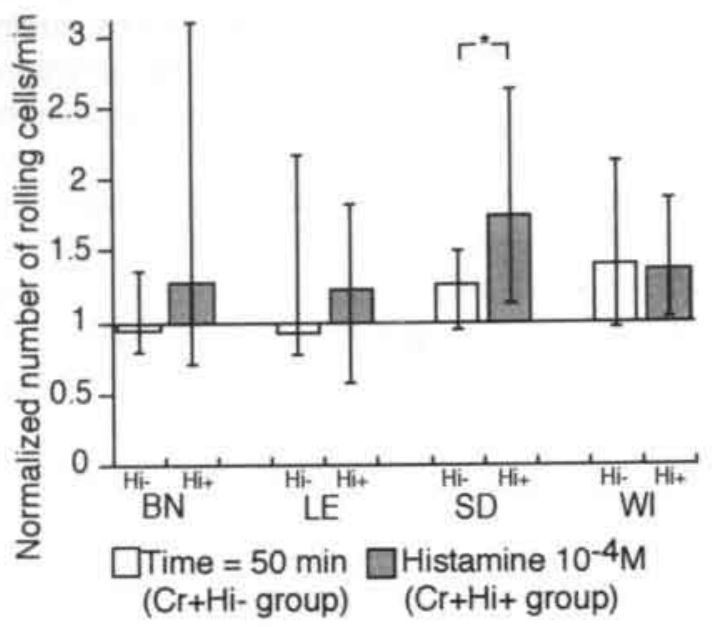

Figure 6.3. Effect of time $\left(\mathrm{Cr}+\mathrm{Hi}\right.$ - group) and administration of $10^{-4} \mathrm{M}$ histamine $(\mathrm{Cr}+\mathrm{Hi}+$ group) on the level of leukocyte rolling in cromoglycate pretreated animals. Medians and interquartile ranges are presented. Data are normalized to baseline values.

$\mathrm{Hi}-=$ no histamine administration; $\mathrm{Hi}+=$ administration of $10^{-4} \mathrm{M}$ histamine $* \mathrm{p} \leq 0.05$ as compared to time control

To enable comparison with data of Kubes and Kanwar (1994) the ratio of the number of rolling leukocytes per minute and the rolling velocity was calculated and expressed in number per $100 \mu \mathrm{m}$ vessel. Baseline values of this parameter without cromoglycate pretreatment were high in $\mathrm{BN}$ (median $1.85 / 100 \mu \mathrm{m} ; \mathrm{p} \leq 0.05$ ) as compared to LE $(0.80 / 100 \mu \mathrm{m})$, SD $(1.36 / 100 \mu \mathrm{m})$, and WI $(1.10 / 100 \mu \mathrm{m})$. No effect of cromoglycate on this parameter was found.

Effect of histamine on leukocyte rolling and adhesion in cromoglycate treated rats Administration of $10^{-5} \mathrm{M}$ histamine after mast cell stabilization did not significantly influence the level and velocity of leukocyte rolling as compared to the time control group. The effect of $10^{-4} \mathrm{M}$ histamine on the level of leukocyte rolling after mast cell stabilization by cromoglycate $(\mathrm{Cr}+\mathrm{Hi}+$ group) as compared to the effect of time $(\mathrm{Cr}+\mathrm{Hi}$ - group) is shown in Figure 6.3. Superfusion with the highest concentration of histamine resulted in a significant increase in the level of leukocyte rolling as compared to time control only in SD. In addition, only in SD $10^{-4} \mathrm{M}$ histamine induced a significant decrease in leukocyte rolling velocity as compared to time control.

Asako and colleagues (Asako et al., 1994) found in Sprague-Dawley rats that histamine induced leukocyte rolling only in venules with a baseline level of less than 20 rolling cells per minute. To analyze whether the observed increase in leukocyte rolling induced by $10^{-4} \mathrm{M}$ histamine in SD rats was equally present in all venules, we plotted a cumulative frequency distribution of the level of leukocyte rolling in all venules of SD rats (Figure 6.4). Each point in the figure represents the number of venules ( $y$-axis) with a level of leukocyte rolling equal to or higher than that indicated 
on the $\mathrm{x}$-axis. Figure $6.4 \mathrm{~A}(\mathrm{Cr}+\mathrm{Hi}$ - group) clearly shows that the level of leukocyte rolling hardly changed with time (no significant differences between curves). Figure $6.4 \mathrm{~B}(\mathrm{Cr}+\mathrm{Hi}+$ group $)$ indicates that with histamine the curve is shifted to the right over a distance that equals a factor of 1.3 for the lowest and 1.9 for the highest concentration, indicating that $10^{-4} \mathrm{M}$ histamine increases the level of leukocyte rolling by a factor of on the average 1.9. This increase is independent of the baseline level of leukocyte rolling, since the shift is similar over the whole range of rolling.

In cromoglycate treated rats without histamine $(\mathrm{Cr}+\mathrm{Hi}$ - group), leukocyte adhesion did not change with time in $\mathrm{BN}$ and $\mathrm{SD}$, but increased significantly in LE (x1.8) and $\mathrm{WI}\left(\times 3\right.$; Figure 6.5A). Only in $\mathrm{BN} 10^{-4} \mathrm{M}$ histamine induced a significant increase in leukocyte adhesion when corrected for the median change in the time control group (Figure 6.5B). In SD this increase was near the level of significance $(p=0.07)$.
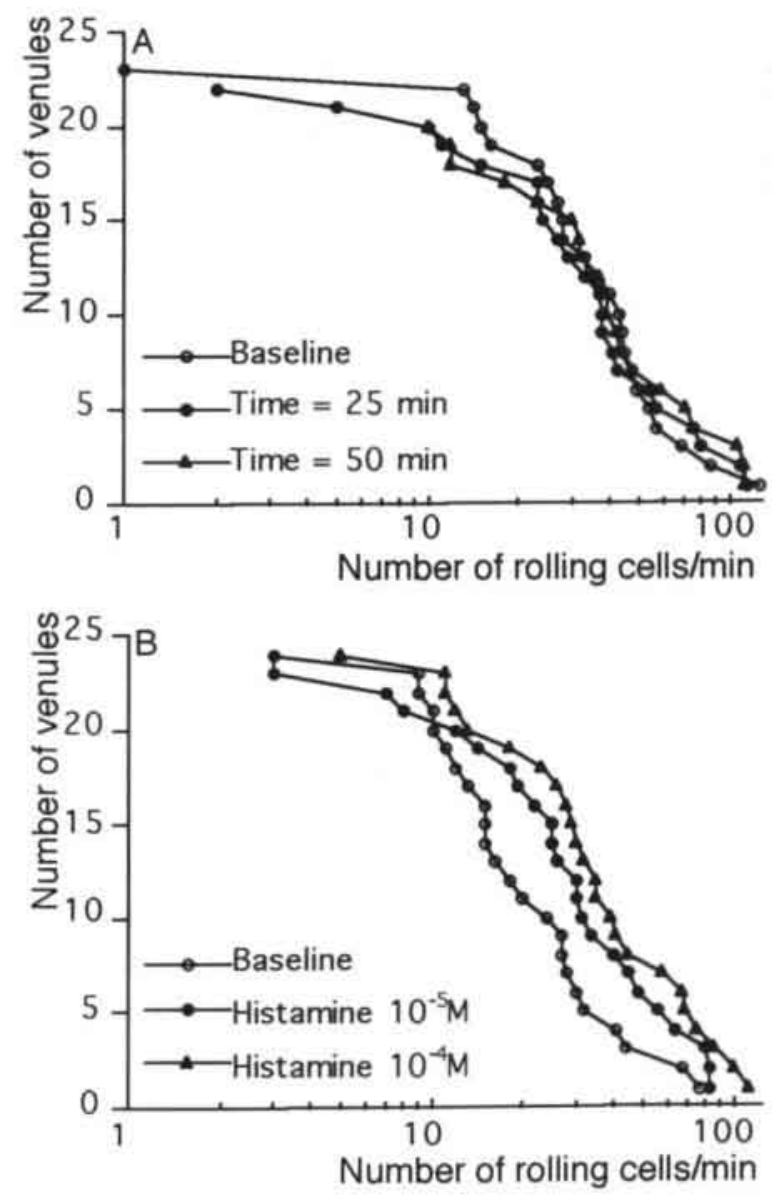

Figure 6.4. Cumulative frequency distribution of the level of leukocyte rolling in all investigated mesenteric venules of Sprague-Dawley rats. A: as a function of time $(\mathrm{Cr}+\mathrm{Hi}$ group), B: as a function of increasing concentrations of histamine ( $\mathrm{Cr}+\mathrm{Hi}+$ group). Each point in the figure represents the number of venules ( $y$-axis) with a level of leukocyte rolling equal to or higher than that indicated on the $x$-axis. 

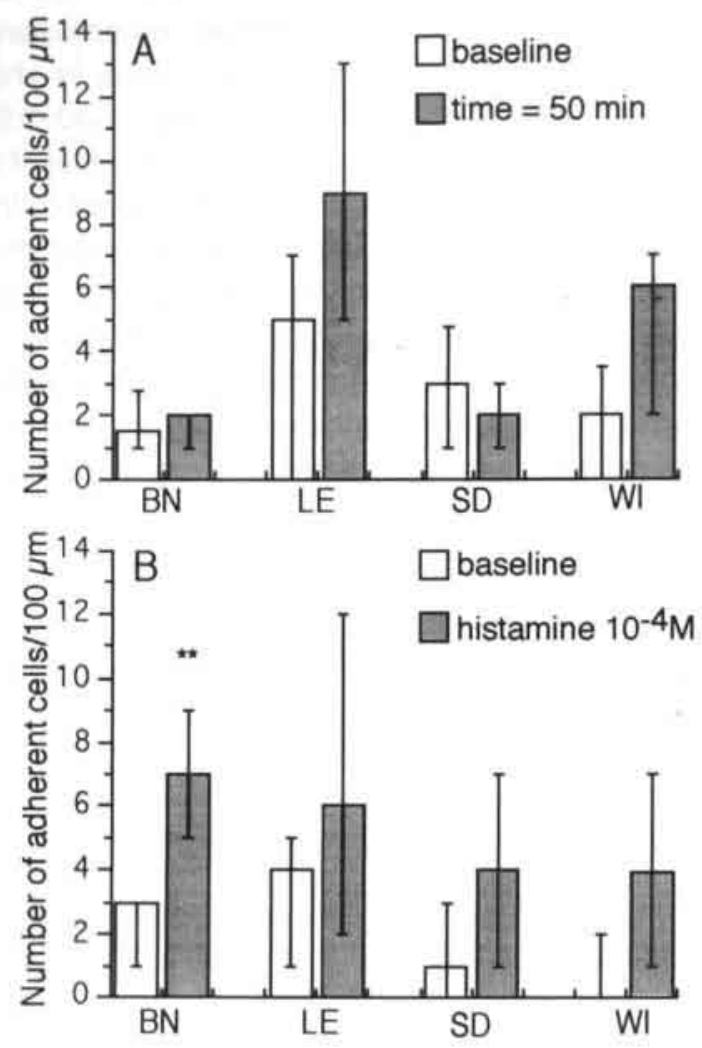

Figure 6.5. Effect of time ( $\mathrm{A}: \mathrm{Cr}+\mathrm{Hi}$ - group) or superfusion with $10^{-4} \mathrm{M}$ histamine $(\mathrm{B}: \mathrm{Cr}+\mathrm{Hi}+$ group) on leukocyte adhesion in cromoglycate pretreated animals. Medians and interquartile ranges are presented.

** $\mathrm{p} \leq 0.01$ as compared to effect of time $(\mathrm{Cr}+\mathrm{Hi}$ - group)

\section{Fluid dynamics}

Baseline fluid dynamic parameters as measured in the selected venules of the $\mathrm{Cr}$ and $\mathrm{Cr}+$ groups are presented in Table 6.1. Median venular diameter did not differ between the experimental groups. No change in venular diameter was seen during histamine superfusion, except for a small increase $(8 \%)$ in SD during superfusion with $10^{-4} \mathrm{M}$ histamine. Baseline RBC velocity and reduced velocity were low in cromoglycate treated $\mathrm{BN}$ rats; reduced velocity was high in cromoglycate treated $\mathrm{WI}$ rats ( $p_{50.05}$ as compared to $\mathrm{Cr}$-group). In some groups small changes in these parameters occurred during histamine superfusion, but no differences were found when compared to time control $(\mathrm{Cr}+\mathrm{Hi}$ - group), indicating that the changes observed following topical administration of histamine are not caused by hemodynamic changes. 
Table 6.1. Baseline fiuid dynamic parameters in mesenteric venules in various rat strains. Median values are presented with interquartile ranges between parentheses.

\begin{tabular}{|c|c|c|c|c|c|c|c|c|}
\hline & \multicolumn{2}{|c|}{ Brown Norway } & \multicolumn{2}{|c|}{ Lewis } & \multicolumn{2}{|c|}{ Sprague-Dawley } & \multicolumn{2}{|c|}{ Wistar } \\
\hline & $\operatorname{Cr}-(n=13)$ & $\mathrm{Cr}+(n=31)$ & $C r-\langle n=15\rangle$ & $\mathrm{Cr}+(n=34)$ & $\mathrm{Cr}-(n=20)$ & $C r+(n=47)$ & $C r-(n=21)$ & $C r+(n=47)$ \\
\hline $\begin{array}{l}\text { Diameter } \\
(\mu \mathrm{m})\end{array}$ & $\begin{array}{c}29 \\
(23-32)\end{array}$ & $\begin{array}{c}24 \\
(22-29)\end{array}$ & $\begin{array}{c}27 \\
\langle 23-29\rangle\end{array}$ & $\begin{array}{c}26 \\
(23-30)\end{array}$ & $\begin{array}{c}24 \\
(22-25)\end{array}$ & $\begin{array}{c}24 \\
(22-27)\end{array}$ & $\begin{array}{c}28 \\
(25-31)\end{array}$ & $\begin{array}{c}26 \\
(23-30)\end{array}$ \\
\hline $\begin{array}{l}\text { RBC vel } \\
\text { (mm/s) }\end{array}$ & $\begin{array}{c}1.7 \\
(1.0-2.0)\end{array}$ & $\begin{array}{c}0.8^{\circ} \\
(0.6-0.9)\end{array}$ & $\begin{array}{c}1.1 \\
(1.0-2.1)\end{array}$ & $\begin{array}{c}1.4 \\
(1.0-1.6)\end{array}$ & $\begin{array}{c}1.6 \\
(1.0-2.0)\end{array}$ & $\begin{array}{c}1.2 \\
(0.9-1.9)\end{array}$ & $\begin{array}{c}1.3 \\
(0.9-1.9)\end{array}$ & $\begin{array}{c}1.6 \\
(1.1-2.5)\end{array}$ \\
\hline $\begin{array}{l}U \\
\left(s^{-1}\right)\end{array}$ & $\begin{array}{c}61 \\
(43-73)\end{array}$ & $\begin{array}{c}29^{\circ} \\
(25-35)\end{array}$ & $\begin{array}{c}44 \\
(33-82)\end{array}$ & $\begin{array}{c}55 \\
(39-64)\end{array}$ & $\begin{array}{c}61 \\
(42-88)\end{array}$ & $\begin{array}{c}52 \\
(38-72)\end{array}$ & $\begin{array}{c}50 \\
(29-63)\end{array}$ & $\begin{array}{c}67^{\circ} \\
(44-92)\end{array}$ \\
\hline
\end{tabular}

$C_{r-}=$ animals nof pretreated with cromoglycale; $\mathrm{Cr}+=$ animals prefreated with cromoglycate; $n=n u m b e r$ of venules; $R B C$ vel = mean red blood cell velocity; $U=$ reduced velocity; " $p \leq 0.05$ as compared to Cr-group 


\subsection{Discussion}

Inflammatory mediators released by mast cells, such as histamine, have been suggested to be involved in the induction of leukocyte-endothelium interactions (Asako et al., 1994; Kanwar and Kubes, 1994; Kubes and Kanwar, 1994; Ley, 1994; Thorlacius et al., 1994; Gaboury et al., 1995). The majority of these studies was performed in one rat strain, namely Sprague-Dawley rats. To investigate whether strain-specific differences exist in this respect, the role of mast cells and of histamine in leukocyte-endothelium interactions was studied in four rat strains. The mast cell stabilizer cromoglycate had no significant effect on baseline level or the velocity of leukocyte rolling in any of the strains. Following mast cell stabilization, exogenously administered histamine increased the level of leukocyte rolling and decreased leukocyte rolling velocity in Sprague-Dawley rats, but not in the other strains; leukocyte adhesion was only significantly increased in Brown Norway rats.

The mast cell stabilizer cromoglycate did not affect the baseline level and velocity of leukocyte rolling in any of the four rat strains, even in Brown Norway rats in which mast cell degranulation was most clearly decreased. In addition, no overall correlation between the percentage of degranulated mast cells around a venule or within the whole mesenteric segment on the one hand and the number of rolling cells on the other could be detected in any of the rat strains (data not shown). Therefore, degranulation of mast cells is not the only factor involved in the induction of leukocyte rolling in rat mesenteric venules. This is in accordance with findings in the rabbit mesentery: in this species leukocyte-endothelium interactions are rapidly induced by exteriorization of the mesentery (Fiebig et al., 1991), although hardly any mast cells are present, and cromoglycate and histamine receptor antagonists do not influence leukocyte-endothelium interactions (Tromp et al., 1996). Apparently, mesenteric mast cell densities differ significantly between various strains and species; human mesentery stained following the same protocol showed a mast cell density of approximately $10 / \mathrm{mm}^{2}$ tissue area (personal observation; data not shown). Therefore, the role of mast cells in the induction of leukocyte rolling, if any, is likely strain- and species-dependent.

The strain-specific role of mast cells in the induction of leukocyte-endothelium interactions is supported by our findings with respect to the effect of exogenously administered histamine. After mast cell stabilization, topical administration of histamine resulted in an increase in the level of leukocyte rolling and a decrease in rolling velocity as compared to time control only in Sprague-Dawley rats. Unlike findings of Asako and colleagues (1994), the increase in leukocyte rolling in Sprague Dawley rats was found in all venules and not only in those with a baseline of less than 20 rolling cells per minute (see Figure 4). Also the influence of histamine on leukocyte adhesion was strain-specific. Only in Brown Norway rats a significant increase in leukocyte adhesion, as compared to the effect of time, was observed.

In our study histamine influenced leukocyte rolling in Sprague-Dawley rats, as reported by Kubes and Kanwar (1994), but not in the other rat strains. The absence of an effect of cromoglycate on leukocyte rolling in Sprague-Dawley rats, however, is 
at variance with their finding that baseline leukocyte rolling is inhibited by $80 \%$ following mast cell stabilization, using the same dose of cromoglycate and the same instant of administration in the protocol. Because the baseline level of leukocyte rolling is comparable in their and our study (about 20 and 30 rolling cells/min, respectively), it is unlikely that the difference is caused by differences in tissue handling. Unfortunately, Kubes and Kanwar did not provide histological data on actual mast cell stabilization, hampering a more detailed comparison between the two studies. Other possible explanations for the difference between the studies might be nutrition, environmental factors and/or genetic factors. Sprague-Dawley, after all, is an outbred strain, and the rats may differ significantly between different colonies.

Our findings indicate that in some strains or species other factors than mast cell degranulation must be involved in the induction of leukocyte-endothelium interactions. Considering the rate of onset of leukocyte rolling, it might be a Pselectin mediated event (Geng et al., 1990). Not only histamine may be involved in the redistribution of P-selectin on the endothelial cell surface, but also other mediators, like thrombin (Hattori et al., 1989), plasmin (Montrucchio et al., 1996), oxygen radicals (Patel et al., 1991), and leukotrienes $\mathrm{C}_{4}$ and $\mathrm{D}_{4}$ (Datta et al., 1995). It is conceivable that any of these mediators is released during the surgical procedure, inducing P-selectin mobilization. So far, it is unknown whether mechanical manipulation per se upregulates $\mathrm{P}$-selectin. It has recently been shown that surgical trauma induces P-selectin expression in the mouse cremaster muscle (Jung and Ley, 1997), but since mast cells were observed in this tissue, it is unknown whether this upregulation of P-selectin was caused by mast cell degranulation or by mechanical manipulation of the tissue.

Although the systemic number of leukocytes did not change in the course of the experiments, the percentage of polymorphonuclear cells (PMNs), the rolling cells in the exteriorized rabbit mesentery (Tangelder et al., 1995), increased substantially, suggesting recruitment of neutrophils. Because the increase in percentage of PMNs was similar in the histamine treated and time control rats of all strains excludes the possibility that changes in the level of leukocyte rolling in the histamine treated rats were caused by an increased delivery of PMNs to the venules under observation.

In conclusion, our study shows that the hypothesis presented in other studies that degranulation of mast cells, and more specifically the release of histamine, is of major importance for the induction of leukocyte-endothelium interactions in rat mesenteric venules may not be generally applicable. The pathway of onset of leukocyte rolling is strain and species dependent. 



REDUCTION IN LEUKOCYTE ROLLING

To be submitted as: S.C. Tromp, K.J.M. Maaijwee, G.J. Tangelder, D.W. Slaaf, R.S. Reneman, J.S. van Velzen, and M.G.A. oude Egbrink. The role of selectins in the thromboembolism induced reduction in leukocyte rolling.

\section{Abstract}

A thromboembolic reaction is known to cause a reduction in leukocyte rolling downstream of the thrombus in rabbit mesenteric venules. The objective of the present study was to explore the possible involvement of selectins in this phenomenon. To this purpose, monoclonal antibodies against L-selectin, P-selectin, or E-selectin, or an isotype control antibody (2 mg/kg i.v.) were administered to anesthetized rabbits in the first hour and in the second hour after exteriorization of the mesentery ( $n=40 ; 8$ groups). Leukocyte rolling was reduced by anti-P-selectin in the first hour and by anti-E- and anti-L-selectin in the second hour. A thromboembolic reaction was induced by vessel wall puncture and leukocyte rolling was assessed upstream and downstream of the thrombus. The thromboembolism induced reduction in leukocyte rolling was more pronounced about one hour (early time phase) than about two hours (late time phase) after tissue exteriorization. In the early time phase half of the reduction disappears in the presence of anti-P-selectin; in the late phase the reduction becomes more pronounced in the presence of anti-Lor anti-E-selectin. We conclude that about one hour after exteriorization of the rabbit mesentery the thromboembolism induced reduction in leukocyte rolling downstream of a thrombus is due to inhibition of P-selectin and adhesion molecules other than the selectins. In the second hour this reduction results from inhibition of other adhesion molecules than the selectins studied, although inhibition of E- and Lselectin cannot be excluded. 


\subsection{Introduction}

Platelet-vessel wall interactions have been shown to influence leukocyteendothelium interactions in vivo. In venules of the rabbit mesentery a thromboembolic reaction as induced by vessel wall injury causes a reduction in leukocyte rolling of about $45 \%$ downstream of the thrombus (oude Egbrink et al., 1992). It was shown that the reduction of leukocyte rolling is not due to the high velocities in the stenosis caused by the thrombus or to capturing of leukocytes within the emboli. The mechanism underlying the thromboembolism induced reduction in leukocyte rolling is as yet unknown.

In the past few years increasing evidence has shown that selectins play an important role in the rolling of leukocytes along the endothelium of post-capillary venules (Tedder et al., 1995; Vestweber and Blanks, 1999). Three of these adhesion molecules have been identified. L-selectin is constitutively present on leukocytes, and can be shed from these cells upon activation (Kansas, 1992). P-selectin is stored in alpha-granules of platelets and in Weibel-Palade bodies of endothelial cells. Within minutes after stimulation of the cell by such mediators as histamine and thrombin, P-selectin can be redistributed to the cell surface (Geng et al., 1990). Eselectin is synthesized and expressed on endothelial cells after stimulation with cytokines; in vitro its maximal expression is found 2 to 4 hours after stimulation (Bevilacqua et al., 1989).

It was the aim of the present study to explore the possible involvement of these selectins in the reduction in leukocyte rolling following a thromboembolic reaction. To this purpose, a thromboembolic reaction was induced in rabbit mesenteric venules in the presence of a monoclonal antibody against each of the three selectins and the level of leukocyte rolling downstream of the thrombus was compared to that upstream. Since the involvement of the three selectins in leukocyte rolling is likely to be time dependent (Ley et al., 1995), we studied the effect of each antibody on leukocyte rolling about one hour (early time phase) and about two hours (late time phase) after exteriorization of the mesentery, both before and after the induction of the thromboembolic reaction. An isotype control antibody was used as control.

\subsection{Materials and methods}

\section{Experimental setup and intravital video microscopy}

The experiments were performed on 40 New Zealand white rabbits (on the average 5 per group) of either sex were used (weight 1.3 to $2.0 \mathrm{~kg}$ ). Anesthesia was induced by intramuscular injection of $40 \mathrm{mg} / \mathrm{kg}$ body wt ketamine hydrochloride (Nimatek, Ad Usem Veterinarium, Cuijk, The Netherlands) and $4 \mathrm{mg} / \mathrm{kg}$ body wt xylazine hydrochloride (Sedamun, Ad Usem Veterinarium, Cuijk, The Netherlands) and was maintained by continuous intravenous infusion of ketamine $(40 \mathrm{mg} / \mathrm{kg} / \mathrm{h})$ and xylazine $(5 \mathrm{mg} / \mathrm{kg} / \mathrm{h}$ ) dissolved in a Lactetrol solution (Aesculaap, Boxtel, The Netherlands; $15 \mathrm{ml} / \mathrm{h}$ ). The drugs were administered through a catheter (PE-50) in the femoral vein. All experimenal protocols were approved by the local ethical 
committee on the use of laboratory animals.

Throughout the experiments body temperature was kept between 37 and $38^{\circ} \mathrm{C}$ by means of an infrared heating lamp controlled by a thermo-analyzer system (Hugo Sachs Elektronik, Hugstetten, Germany) connected to a rectal probe. Arterial blood pressure was measured continuously through a catheter (PE-50) in the femoral artery (Uniflow external pressure transducer, Baxter, Santa Ana, CA, U.S.A.). Heart rate was assessed from the instantaneous pressure signal. To keep the arterial catheter patent, it was continuously perfused with physiological saline $(3 \mathrm{ml} / \mathrm{h})$ via the Uniflow system; no heparin was used. To maintain systemic arterial $\mathrm{pH}, \mathrm{pCO}_{2}$, and $\mathrm{pO}_{2}$ at normal values, the rabbits were ventilated throughout the experiments. Ventilation with a positive end-expiratory pressure of $2 \mathrm{~cm} \mathrm{H}_{2} \mathrm{O}$ was performed through a tracheal tube ( 3.5 or $4.5 \mathrm{~mm}$ i.d., Mallinckrodt $\mathrm{GmbH}$, Hennef, Germany) connected to an infant ventilator (Loosco, model mk 2, Hoek Loos, Amsterdam, The Netherlands). The rabbits were ventilated with a mixture of oxygen $(25 \%)$, nitrogen $(74 \%)$ and carbon dioxide (1\%). Respiration frequency was $60 /$ minute and, depending on the weight of the rabbit, tidal volume varied between 9 and $14 \mathrm{ml}$. Blood gas and $\mathrm{pH}$ values were measured in blood collected from a central ear artery, using an acid-base analyzer (ABL 3, Radiometer, Copenhagen, Denmark). Mean values ( \pm s.d.) were: $\mathrm{pH} 7.38 \pm 0.49, \mathrm{pCO}_{2} 40.6 \pm 4.9 \mathrm{mmHg}$, and $\mathrm{pO}_{2} 95.7 \pm 16.1$ $\mathrm{mmHg}$.

Through a small abdominal midline incision a segment of the distal ileum was exteriorized. The mesentery was carefully spread over a siliconized glass plate mounted in an electrically heated microscope table $\left(37^{\circ} \mathrm{C}\right)$. The preparation was continuously superfused with buffered Tyrode's solution $\left(37^{\circ} \mathrm{C} ; \mathrm{pH} 7.35-7.40\right)$. The bowels were kept moist with overlying wet gauze. Mesenteric venules were visualized with a Leitz intravital microscope, adapted for telescopic imaging (Slaaf et al., 1982), and a Leitz water-immersion objective (SW25, numerical aperture 0.60). Transillumination was performed with a tungsten lamp. Images were recorded on videotape (Sony Betamax or Panasonic SVHS) through a CCD camera (Hamamatsu, C2400) or a Grundig TV camera (model FA 32). Final magnification at the front plane of the camera was $\times 52$.

\section{Experimental protocols}

After exteriorization of the mesentery the tissue was allowed to stabilize. Stabilization lasted 30 minutes in the early groups and 75 minutes in the late groups (see Figure 7.1). After selection of the venules (total $n=150$ ), each venule ( 1 to 5 per animal) was recorded on video for at least 2 minutes and red blood cell (RBC) velocities were measured on-line (see below). Subsequently, one of the antibodies against $\mathrm{P}_{-}, \mathrm{L}$-, or E-selectin or the isotype control antibody $(2 \mathrm{mg} / \mathrm{kg}$ ) was injected into the marginal ear vein in 1-2 minutes. Within 2-15 minutes following injection of the antibody each selected venule was recorded again for 2 minutes to evaluate the effect of the antibody on leukocyte rolling and on the local fluid dynamic conditions. To allow adequate detection of changes in leukocyte rolling induced by the monoclonal antibodies, if any, only venules with 5 or more rolling leukocytes per 
minute after 30 minutes of stabilization were used. Hardly any vessels had to be excluded.

Subsequently, 15 to 90 minutes after antibody administration a thromboembolic reaction was induced in 23 of the 40 rabbits in a total of 54 venules. To induce a thromboembolic reaction, the vessel wall was punctured with a glass micropipette (tip diameter 6-8 $\mu \mathrm{m}$ ), as previously described (oude Egbrink et al., 1988). Puncture was considered to be successful only if red blood cells could be seen leaving the vessel; the duration of this bleeding was short (median: 7 seconds). After puncture the thromboembolic reaction started at once: a thrombus was formed within a second and, subsequently, emboli were produced on the downstream side of the thrombus in most vessels. Leukocyte rolling was assessed in the first 2 minutes following puncture at 3 sites: immediately upstream of the thrombus, immediately downstream $(0-40 \mu \mathrm{m})$, and further downstream $(60-100 \mu \mathrm{m})$ of the thrombus. All three sites were visible on the monitor at the same time.

\section{Experimental parameters}

Vascular diameters (range: $15-36 \mu \mathrm{m}$ ) were measured off-line with an image shearing device (Intaglietta and Tompkins, 1973), while red blood cell (RBC) velocity was determined on-line using a prism grating system with the slit covering the whole vessel width (Slaaf et al., 1981). To obtain actual mean RBC velocities, the measured velocity values were divided by a conversion factor of 1.1 (Slaaf et al., 1986; Tangelder et al., 1986). Reduced velocity (mean RBC velocity/vessel diameter), which is a measure of wall shear rate, was calculated from these parameters.

Leukocytes were considered to be rolling if they could be seen moving along the vessel wall by eye, either at a rather constant velocity or saltating at a significantly lower rate than the blood was flowing. To assure that all rolling leukocytes could be distinguished, the midplane of a venule was kept in focus (oude Egbrink et al., 1992). Both the level of leukocyte rolling and the rolling velocity were determined. Leukocyte rolling level was quantitated off-line by counting in duplicate the number of cells that rolled through a segment of the venule during a period of 100 to $120 \mathrm{~s}$; it was expressed as the number of rolling cells passing per minute. The length of the segment was approximately equal to the diameter of the vessel. The velocity of the rolling leukocytes was determined off-line by measuring the time it took 10 randomly chosen rolling leukocytes to travel a certain distance along the vessel wall.

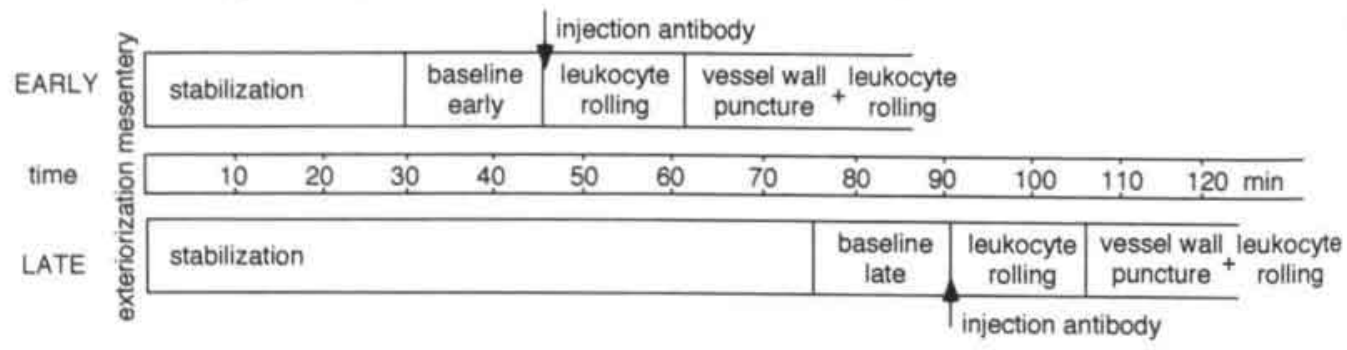

Figure 7.1. Experimental protocol 
To evaluate the effects of the antibodies on embolization, if any, the total duration of embolization, i.e., the time between puncture and the production of the last embolus, and the number of emboli produced were determined off-line from videotape. To evaluate effects on thrombus stability, the rebleeding frequency was determined. The observation time per vessel was $600 \mathrm{~s}$ from the moment of puncture.

Systemic leukocyte counts were determined at the beginning of the stabilization period, and in the middle and at the end of each experiment. To this purpose, $20 \mu \mathrm{l}$ blood was collected from a central ear artery and added to Türks solution $(0.2 \mathrm{mg}$ gentian violet in $1 \mathrm{ml}$ glacial acetic acid, $6.25 \% \mathrm{vol} / \mathrm{vol}$ ) in a 1:10 dilution; leukocytes were counted and differentiated as polymorphonuclear (PMN) or monomorphonuclear (MMN) in a counting chamber (Clay Adams, Parsippany, N.J., U.S.A.). Of these two leukocyte subclasses the PMNs are the ones that are rolling in rabbit mesenteric venules (Tangelder et al., 1995).

\section{Monoclonal antibodies}

The monoclonal antibodies used were anti-rabbit L-selectin (DREG-200; see (Kishimoto et al., 1990; von Andrian et al., 1991)), anti-rabbit P-selectin (PRAB), anti-rabbit E-selectin (ERAB), and an isotype mouse lgG1 as control (EP5C7). All monoclonal antibodies were kindly provided by Dr. E.L. Berg. Protein Design Labs, Inc. (Mountain view, CA, U.S.A.) and stored at $4^{\circ} \mathrm{C}$. On the day of the experiment they were diluted in sterile saline to concentrations of $2 \mathrm{mg} / \mathrm{ml}$ for injection.

\section{Data analysis and statistics}

Because of their nonsymmetrical distribution, data are presented as medians with interquartile ranges (i.e., the spread from 25 th to 75 th percentile). To enable comparisons between groups, some data are presented normalized to their baseline values. Paired data groups were compared using the Wilcoxon signed-rank test. Differences between two independent data groups were tested with the MannWhitney $\mathrm{U}$ test; to compare more than two independent data groups the KruskalWallis test was used. Correlations were performed by using the Spearman test. In all tests, the level of significance was set at 0.05 .

Table 7.1. baseline levels of leukocyte rolling in number of cells per minute in the various groups before antibody administration. Medians are presented with interquartile ranges between parentheses.

\begin{tabular}{lllll}
\hline & isotype & anti-L-selectin & anti-P-selectin & anti-E-selectin \\
\hline early: & $24(17-40)$ & $30(25-36)$ & $33(21-43)$ & $20(14-32)$ \\
& $n=21$ & $n=24$ & $n=24$ & $n=14$ \\
late: & $19(13-23)$ & $11(6-20)$ & $13(10-24)$ & $19(14-22)$ \\
& $n=18$ & $n=17$ & $n=13$ & $n=19$ \\
\hline
\end{tabular}

control $=$ isotype monoclonal antibody; $n=$ number of venules 


\subsection{Results}

\section{Role of selectins in leukocyte rolling over time}

Table 7.1 shows the median baseline number of rolling leukocytes per minute, i.e., before antibody administration, in all groups and the number of venules studied in the various groups. There were no significant differences between the baseline values in the four early and in the four late groups, but the baseline values were lower in the late than in the early groups $(p<0.001)$.

In the early time phase (Figure $7.2 \mathrm{~A}$ ) anti-P-selectin was the only monoclonal antibody with a nearly significant influence $(p=0.1)$ on leukocyte rolling as compared to the reduction in leukocyte rolling as induced by the isotype control antibody (37\% versus $20 \%$ ). Anti-L-selectin and anti-E-selectin had no effect on baseline leukocyte rolling. In the second hour after exteriorization, however, (Figure 7.2B), anti-Pselectin had no effect, but anti-L-selectin and anti-E-selectin reduced leukocyte rolling significantly, by $55 \%(p=0.001)$ and $27 \%(p=0.02)$, respectively, as compared to the reduction in the control situation (3\%).

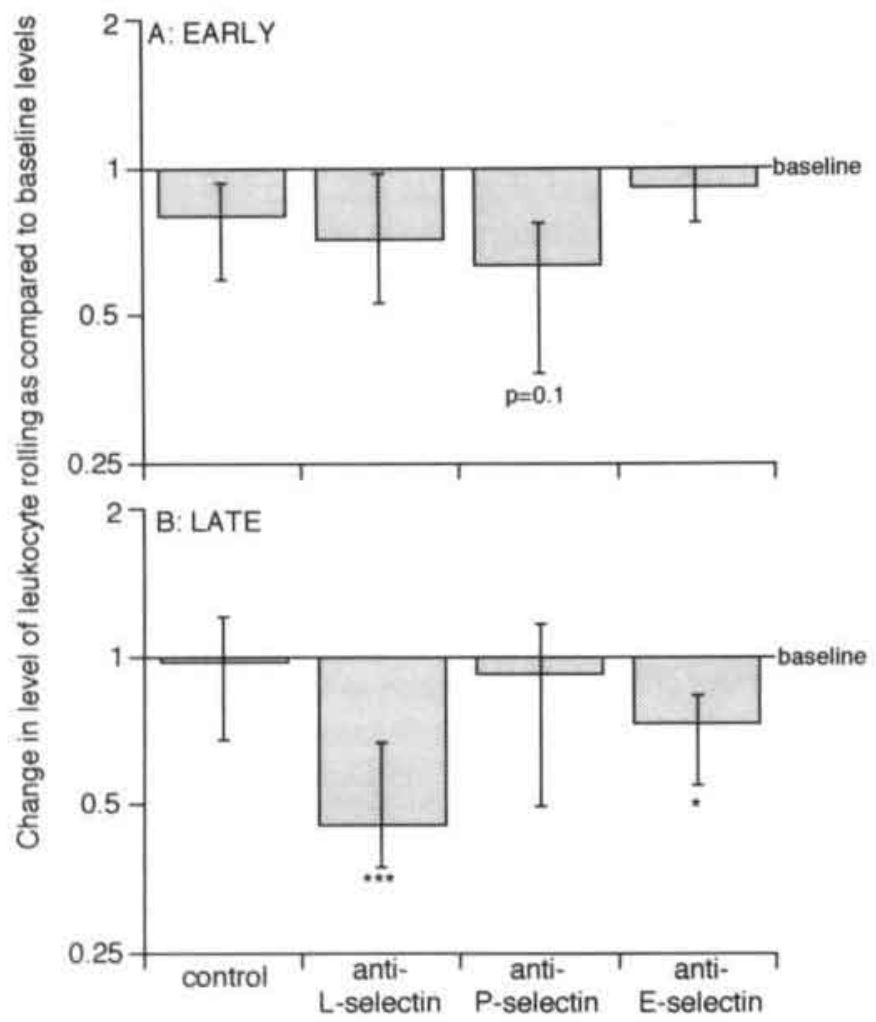

Figure 7.2. Effect of (A) early (about one hour after exteriorization of the rabbit mesentery) or (B) late (about two hours after exteriorization of the rabbit mesentery) administration of antibodies $(2 \mathrm{mg} / \mathrm{kg}$ ) on the level of leukocyte rolling as normalized to baseline values (see Table 7.1). Medians and interquartile ranges are shown.

${ }^{\circ} \mathrm{p} \leq 0.1,{ }^{*} \mathrm{p} \leq 0.05,{ }^{* *} \mathrm{p} \leq 0.001$ as compared to control; control = isotype control antibody 


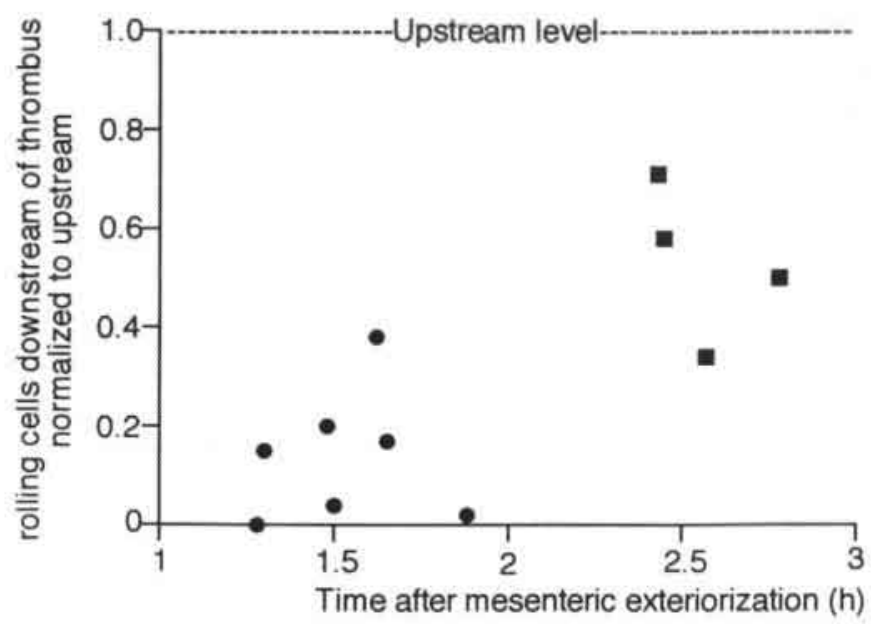

Figure 7.3. Change in level of leukocyte rolling immediately downstream of a thrombus normalized to the level upstream $(=1)$ in the two control groups early and late administration of the isotype control antibody), as related to the time after exteriorization of the mesentery.

Median baseline leukocyte rolling velocity ranged from 26 to $38 \mu \mathrm{m} / \mathrm{s}$ in the early groups and from 41 to $51 \mu \mathrm{m} / \mathrm{s}$ in the late groups. The values were higher in the late than in the early groups $(p<0.001)$, but there were no significant differences between the baseline values within the four early or within the four late groups. None of the anti-selectin antibodies had a significant effect on rolling velocity as compared to the effect of the isotype control antibody, neither in the early nor in the late groups.

\section{Selectins and the effect of thromboembolism on leukocyte rolling}

Figure 7.3 shows the level of leukocyte rolling immediately downstream $(0-40 \mu \mathrm{m})$ of a thrombus as normalized to upstream values in the two control groups (early and late administration of the isotype control antibody). The figure clearly shows that the effect of a thromboembolic reaction on leukocyte rolling changes with time: early after exteriorization of the mesentery the reduction of leukocyte rolling downstream of the thrombus is greater than in the late phase (correlation coefficient between the time after exteriorization on the one hand and the reduction in leukocyte rolling on the other: $0.67 ; p=0.02$ ).

In the early time phase (Figure 7.4A), anti-P-selectin significantly attenuated the reduction in leukocyte rolling immediately behind a thrombus $(p=0.02$ vs. control): the anti-P-selectin effect was still present further downstream of the thrombus $(p=0.05)$. Anti-L-selectin and anti-E-selectin did not significantly alter the control reduction (data not shown). In the late time phase (Figure 7.4B), anti-E-selectin significantly increased the normal thromboembolism induced reduction in leukocyte rolling ( $p=0.05$ vs. control), leaving a median value of only 2 rolling leukocytes per minute. Anti-L-selectin had a similar effect, but this did not reach the level of significance $(p=0.09)$. Anti-P-selectin did not affect the reduction immediately 
downstream of the thrombus (data not shown).

In all groups, the reduction in leukocyte rolling diminished with increasing distance from the thrombus: the reduction was greater between 0-40 $\mu \mathrm{m}$ than between $60-100 \mu \mathrm{m}$ downstream of the thrombus $(p<0.001)$.

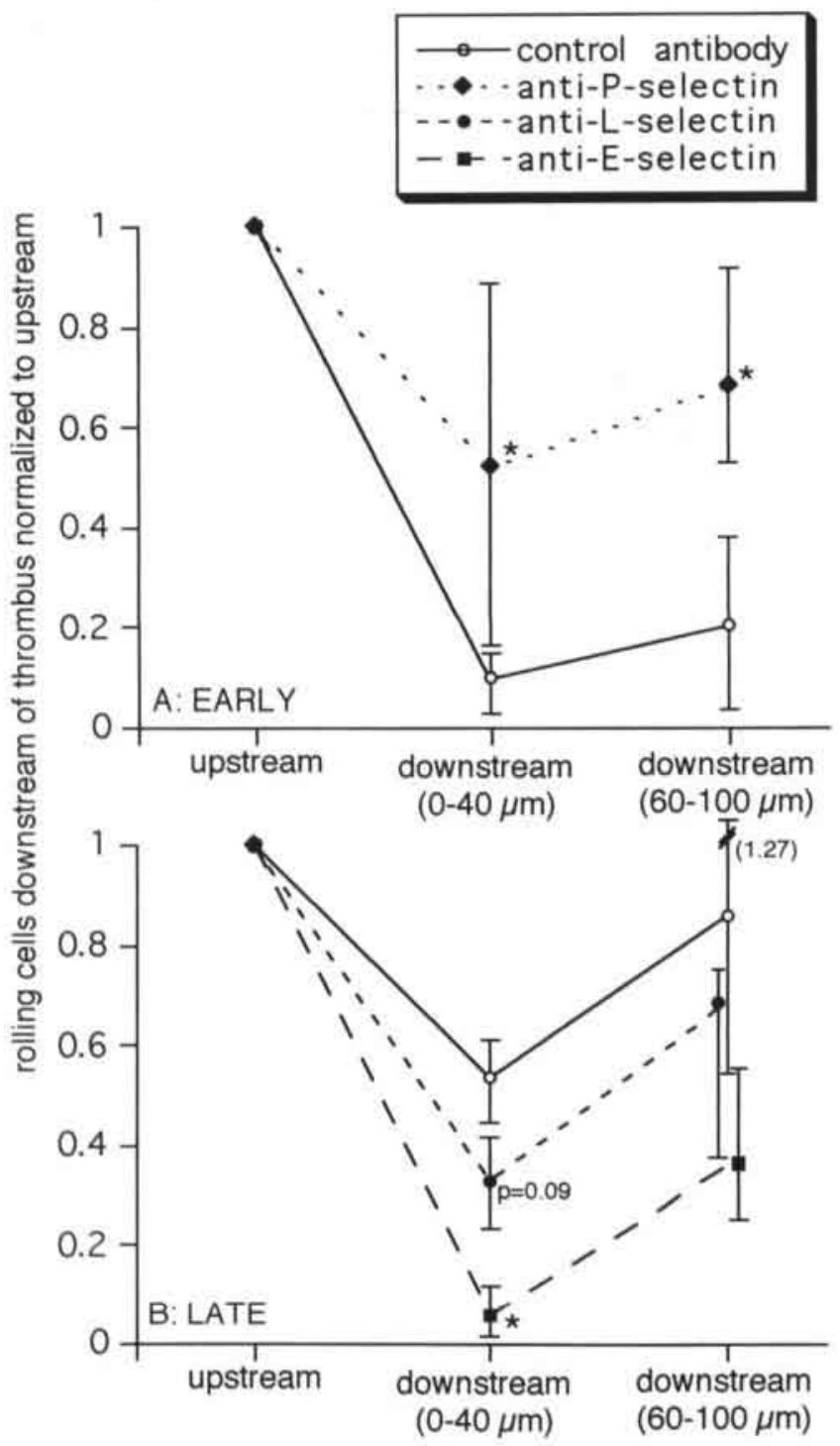

Figure 7.4. Change in level of leukocyte rolling at two sites downstream of a thrombus normalized to the level upstream, in the presence of an antibody against those selectins that are involved in leukocyte rolling in the early (A) or late (B) time phase after exteriorization of the mesentery. Medians and interquartile ranges are presented.

* ps0.05 as compared to corresponding control group 
Effect of anti-selectin antibodies on the thromboembolic reaction

Embolization in the presence of the isotype control antibody was comparable to embolization in the absence of antibodies (Broeders et al., 1998): a median number of 2 emboli was produced per venule in a period of $48 \mathrm{~s}$ (medians). Anti-L-selectin and anti-P-selectin had no significant effect on the embolization parameters as compared to the isotype control antibody, in both the early and the late time phase (Figure 7.5). Anti-E-selectin, however, increased the number of emboli produced $(p=0.06)$ and the duration of embolization $(p=0.09)$ in the late time phase (closed squares); it had no effect in the first hour after stabilization (open squares).

Whereas anti-P-selectin did not influence embolization, it decreased the stability of the thrombus: rebleeding frequency increased almost 4 -fold as compared to control ( $29 \%$ vs. $8 \%$ of the vessels). Anti-L-selectin and anti-E-selectin had no such effect.

\section{Fluid dynamics and systemic leukocyte counts}

The overall median diameter in the venules under investigation was $22 \mu \mathrm{m}$ (interquartile range: $20-25 \mu \mathrm{m}$ ), the median RBC velocity was $1.1 \mathrm{~mm} / \mathrm{s}(0.8-1.6$ $\mathrm{mm} / \mathrm{s})$, and the median reduced velocity $54 \mathrm{~s}^{-1}\left(37-73 \mathrm{~s}^{-1}\right)$. No differences in these parameters existed between the groups before administration of the antibodies. None of the antibodies induced changes in these fluid dynamic parameters, except

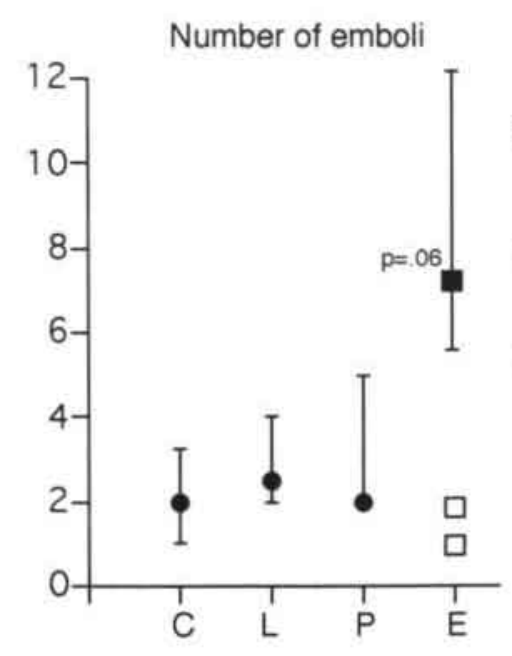

Duration of embolization (s)

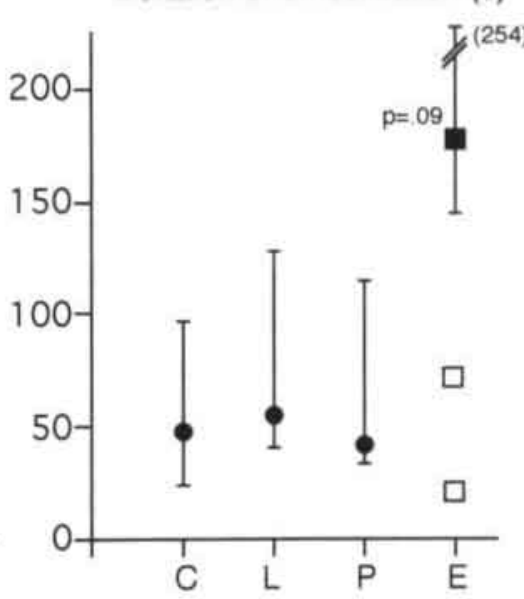

Figure 7.5. The effect of antibodies against selectins on embolization in venules. The total duration of embolization and the number of emboli produced per vessel are shown. Data obtained in the 'early' and 'late' time phase are pooled, since no differences with the corresponding control groups were found. In contrast, results from the animals receiving anti-E-selectin are presented separately ( $\square$ early and $\mathbf{E}$ late administration of anti-E. selectin). Data are presented as medians with interquartile ranges, except for the 'early' anti-E-selectin group, in which only 2 vessels could be measured.

$C=$ isotype control antibody; $L=$ anti-L-selectin antibody; $P=$ anti-P-selectin antibody; $E=$ anti-E-selectin antibody 
for a small significant increase in RBC velocity when anti-L-selectin was administered in the late phase (median $1.5 \mathrm{~mm} / \mathrm{s}$ versus $1.1 \mathrm{~mm} / \mathrm{s}$ in the control group). However, no significant relations were found between RBC velocities and changes in leukocyte rolling, indicating that the observed changes in leukocyte rolling are not caused by hemodynamic changes.

Baseline mean arterial pressure and heart rate were $70 \mathrm{mmHg}$ (interquartile range: $61-75 \mathrm{mmHg}$ ) and 151 beats per minute (138-165 bpm), respectively. No changes in these parameters were observed during the experiments.

In all rabbits, both the systemic number of leukocytes (median $6.5 \times 10^{6} / \mathrm{ml}$; interquartile range $5.5-7.9 \times 10^{6} / \mathrm{ml}$ ) and the percentage of polymorphonuclear (PMN) cells $(40 \% ; 36-45 \%)$ were within normal ranges before the experiments started (Kozma et al., 1974). In each group the systemic number of leukocytes decreased slightly during the experiments, but increased again towards the end, as did the percentage of PMNs. No significant differences were found between the groups, i.e., administration of antibodies did not influence the changes in systemic number of leukocytes nor the percentage of PMNs.

\subsection{Discussion}

The findings of the present study show that the thromboembolism induced reduction in leukocyte rolling is more pronounced about one hour (early time phase) than about two hours (late time phase) after exteriorization of the rabbit mesentery. In the early time phase about half of this reduction disappears in the presence of a monoclonal antibody against P-selectin, but not in the presence of monoclonal antibodies against $\mathrm{E}$ - and L-selectin. In the late time phase the reduction becomes more pronounced in the presence of anti-E-selectin or anti-L-selectin. The embolization is not influenced by antibodies against P-or L-selectin, but in the late time phase the duration of embolization and the number of emboli produced are increased in the presence of anti-E-selectin. The stability of the thrombus is reduced in the presence of anti-P-selectin.

About one hour after exteriorization of the rabbit mesentery P-selectin, but not Land E-selectin, appears to be involved in leukocyte rolling (figure 7.2). In this early time phase the thromboembolism induced reduction in leukocyte rolling is very pronounced $( \pm 90 \%)$. This reduction is almost halved, to $48 \%$, in the presence of antiP-selectin (figure 7.4A), which had no effect on the embolization process itself. The relative increase in leukocyte rolling downstream of the thrombus in the presence of anti-P-selectin as compared to the control situation has to be explained by a decrease in leukocyte rolling upstream of the thrombus and a low contribution of Pselectin to leukocyte rolling downstream of the thrombus. Together, these findings suggest that in this early time phase the thromboembolism induced reduction in leukocyte rolling downstream of the thrombus is partly due to inhibition of P-selectin and partly due to inhibition of adhesion molecules other than the selectins investigated.

In the late time phase L-and E-selectin mediate leukocyte rolling, while $\mathrm{P}$-selectin 
is not involved anymore (figure 7.2). The thromboembolism induced reduction in leukocyte rolling during this period becomes more pronounced in the presence of anti-E- or anti-L-selectin, with E-selectin blockade causing an almost maximal reduction (Figure $7.4 \mathrm{~B}$ ). This indicates that during this late time phase the leukocytes that still roll downstream of a thrombus depend on L-selectin and, more predominantly, on E-selectin. In this light the thromboembolism induced reduction in leukocyte rolling in the late phase could be explained by downregulation of adhesion molecules other than these selectins or their ligands, although a partial downregulation of L- or E-selectin cannot be excluded. One should realize, however, that in the presence of anti-E-selectin the increased embolization may have contributed to the enhanced reduction in leukocyte rolling.

Following a thromboembolic reaction leukocyte rolling is most prominently reduced immediately behind the thrombus; the effect declines further downstream. This reversibility suggests a local effect of the thromboembolic reaction on the endothelium rather than on the leukocytes. Therefore, the adhesion molecules of other families than selectins, possibly involved in the thromboembolism induced reduction of leukocyte rolling, are likely to be localized on the endothelium. Intercellular adhesion molecule-1 (ICAM-1), which is known to support leukocyte rolling as well (Kunkel et al., 1996; Steeber et al., 1998), may be such a molecule.

The mechanism behind the thromboembolism induced, quick reduction in bioavailability of P-selectin and adhesion molecules other than selectins for leukocyte rolling is unknown. From in vitro studies it is known that P-selectin cannot only be rapidly released, but also be rapidly cleared from the cell surface by endocytosis (Subramaniam et al., 1993; Vestweber and Blanks, 1999). Another mechanism for rapid reduction of bio-availability of adhesion molecules is shedding from the cell surface. Either mechanism may have been induced by substances released during the thromboembolic reaction.

The fact that about one hour after exteriorization the reduction in leukocyte rolling downstream of the thrombus is more pronounced than after about two hours, may be explained by a higher sensitivity of P-selectin as compared to L-and E-selectin to (substances released during) the thromboembolic reaction. The fact that in the control situation the reduction in leukocyte rolling in the late time phase is similar to that in the early time phase in the presence of anti-P-selectin supports this notion.

The finding in the present study that in the rabbit mesentery P-selectin is involved in leukocyte rolling early after exteriorization of the tissue, whereas L-selectin plays a role in leukocyte rolling in a later time phase is consistent with similar findings in the mouse cremaster muscle (Ley et al., 1995). The early involvement of P-selectin in leukocyte rolling in rabbit mesentery supports our view that P-selectin can be upregulated independent of mast cell degranulation (Tromp et al., 1998), since hardly any mast cells are present in the rabbit mesentery (Tromp et al., 1996). Interestingly, E-selectin appears to be involved in leukocyte rolling already in the second hour after tissue exteriorization. Up till now expression of E-selectin in rabbit mesenteric venules has only been demonstrated 4 hours after intraperitoneal injection of interleukin-1 (Olofsson et al., 1994). The expression of E-selectin early 
after tissue handling is not necessarily in contradiction with in vitro data suggesting maximal expression of E-selectin 2 to 4 hours after stimulation (Bevilacqua et al., 1989).

A surprising finding in the present study is the nearly significant effect of the Eselectin antibody on embolization in the late time phase. No such effect is apparent in the early time phase, suggesting that the effect of this antibody depends on the expression of E-selectin on the endothelium. In the last few years it has become evident that selectins not only function as binding molecules but also have signaling functions (Crockett-Torabi, 1998; Vestweber and Blanks, 1999). It is possible that binding of structures to E-selectin alters, for example, the endothelial production of anti- and/or prothrombotic substances.

The observation that P-selectin blockade does not influence embolization but decreases thrombus stability, suggests that P-selectin expression on activated platelets does play a role during thrombus formation, but not during embolization. This may be explained by platelet stimulation through strong agonists in thrombus formation and through weak agonists in embolization, because in vitro studies have shown that P-selectin blockade inhibits platelet aggregation induced by a strong platelet agonist, such as collagen (Parmentier et al., 1991), which induces P-selectin redistribution to the cell surface (Isenberg et al., 1986), whereas it does not affect platelet aggregation induced by a weak agonist, such as adenosine diphosphate (ADP) (Boukerche et al., 1996). This view fits with our previous electron microscopic observation that a thrombus consists of tightly packed platelets, which have lost their discoid shape and most of their contents, whereas platelets forming emboli have not lost their shape, and are not degranulated (oude Egbrink, 1989).

In conclusion, about one hour after exteriorization of the rabbit mesentery the thromboembolism induced reduction in leukocyte rolling is due to a decreased bioavailability of P-selectin and of endothelial adhesion molecules for leukocyte rolling other than selectins. Two hours after tissue exteriorization, when P-selectin is not involved any longer endothelial adhesion molecules other than P-selectin are affected by the thromboembolic reaction, although a partial reduction of E- and Lselectin cannot be excluded. 


\section{THE INFLUENCE OF PROSTAGLANDINS ON LEUKOCYTE-ENDOTHELIUM INTERACTIONS}

In press as: S.C. Tromp, G.J. Tangelder, D.W. Slaaf, R.S. Reneman, J.S. van Velzen, and M.G.A. oude Egbrink. The influence of prostaglandins on leukocyteendothelium interactions in rabbit mesenteric venules. Prostaglandins and other lipid mediators (1999)

\section{Abstract}

Contradictory results have been reported concerning the effects of prostaglandins (PGs) on leukocyte-endothelium interactions. Therefore, we investigated the in vivo effects of $\mathrm{PGE}_{1}, \mathrm{PGE}_{2}$, lloprost (a stable $\mathrm{PGI}_{2}$-analogue), and also of a combination of these PGs on leukocyte rolling and FMLP-induced leukocyte adhesion in venules of rabbit mesentery. This preparation was used because of its low level of vasoactivity, eliminating hemodynamic effects on leukocyte-endothelium interactions. The mesentery was superfused with PGs or vehicle. After 30 minutes FMLP was added to the PG-solution for 15 minutes, whereupon the tissue was superfused with the PG-solution alone for another 30 minutes. Neither the PGs nor the cocktail influenced leukocyte rolling. During FMLP administration leukocyte adhesion increased and leukocyte rolling decreased; adhesion was highest in the presence of $\mathrm{PGE}_{2}$. The FMLP-induced decrease in leukocyte rolling was similar in all groups. After FMLP administration had been stopped the number of adherent cells almost returned to baseline and the level of leukocyte rolling increased, the baseline level being reached only in the presence of $\mathrm{PGE}_{2}$. In conclusion, these findings indicate that the effects of PGs on leukocyte-endothelium interactions are limited. 


\subsection{Introduction}

Prostaglandins (PGs) or their chemical analogues have been shown to be beneficial to patients with diseases such as peripheral arterial occlusive disease (U.K., 1991; Altstaedt et al., 1993; Staben and Albring, 1996), chronic heart failure (Pacher et al., 1997), and Raynaud's syndrome (Wigley et al., 1994; Belch et al., 1995). In addition, PGs are effective in limiting the injury following experimentally induced ischemia and reperfusion (Simpson et al., 1987; Simpson et al., 1988; Thomson et al., 1994; Bengisun et al., 1997; Natori et al., 1997). The PGs are thought to exert their overall influence through several effects: vasodilation, reduction of platelet aggregation and inhibition of leukocyte function (Schrör, 1985; Gerritsen, 1996). The reported effects of PGs on leukocyte functions are, however, variable. Contradictory results have been reported on their influence on the interaction of leukocytes with endothelial cells: inhibiting as well as stimulating effects of different PGs on the various steps from leukocyte rolling to leukocyte migration into the tissue have been demonstrated. Most of these studies have been performed in vitro on macrovascular endothelial cells.

In vivo experiments previously performed in our laboratory indicate that $\mathrm{PGs}$ other than thromboxane $A_{2}$ may be involved in the reduction of leukocyte rolling as observed following a thromboembolic reaction in venules of the rabbit mesentery (oude Egbrink et al., 1992). This tissue can be easily used to investigate in vivo leukocyte rolling and leukocyte adhesion to the wall of post-capillary venules, which are both prerequisite steps for subsequent diapedesis and migration into inflamed or injured tissue (von Andrian et al., 1992; Springer, 1994). The mesentery is especially suited to study leukocyte-endothelium interaction because of its low level of vasoactivity (Broeders et al., 1998), allowing the assessment of the influence of agents on these interactions without interference of changes in fluid dynamic conditions. We investigated in venules of the rabbit mesentery in vivo whether different prostaglandins, alone or in combination, are able to affect (1) leukocyte rolling and (2) chemo-attractant induced leukocyte adhesion. As chemo-attractant the peptide $\mathrm{N}$-formyl-methionyl-leucyl-phenylalanine (FMLP) was used. To prevent systemic hemodynamic changes (i.e., blood pressure effects), the PGs were administered locally through topical superfusion of the mesenteric segment under observation. The PGs used were $\mathrm{PGE}_{1}, \mathrm{PGE}_{2}$, and the stable $\mathrm{PGI}_{2}$ analogue lloprost. $\mathrm{PGE}_{1}$ and $\mathrm{PGE}_{2}$ were included, because microvascular endothelial cells mainly produce PGs of the E-series (Gerritsen and Cheli, 1983; Linssen et al., 1993). Since $P \mathrm{PG}_{2}$ is more commonly applied in vascular diseases, this $P G$ was included as well. Since microvascular endothelial cells can produce more than one PG at a time (Gerritsen and Cheli, 1983; Linssen et al., 1993), the effect of a combination of $\mathrm{PGE}_{1}, \mathrm{PGE}_{2}$ and lloprost on leukocyte-endothelium interactions was also examined in this study. 


\subsection{Materials and methods}

\section{Experimental setup and intravital video microscopy}

All experiments were approved by the local ethical committee on the use of laboratory animals. Twenty-nine New Zealand white rabbits of either sex were used (weight 1.6 to $2.5 \mathrm{~kg}$ ). Anesthesia was induced by intramuscular injection of 40 $\mathrm{mg} / \mathrm{kg}$ body wt ketamine hydrochloride (Nimatek, Ad Usem Veterinarium, Cuijk, The Netherlands) and $4 \mathrm{mg} / \mathrm{kg}$ body wt xylazine hydrochloride (Sedamun, Ad Usem Veterinarium, Cuijk, The Netherlands) and maintained by continuous intravenous infusion of ketamine $(40 \mathrm{mg} / \mathrm{kg} / \mathrm{h})$ and xylazine $(5 \mathrm{mg} / \mathrm{kg} / \mathrm{h})$ dissolved in a Lactetrol solution (Aesculaap, Boxtel, The Netherlands; $15 \mathrm{ml} / \mathrm{h}$ ). The drugs were administered through a catheter (PE-50) in the femoral vein.

Throughout the experiments body temperature was kept between 37 and $38^{\circ} \mathrm{C}$ by means of an infrared heating lamp controlled by a thermo-analyzer system (Hugo Sachs Elektronik, Hugstetten, Germany) connected to a rectal probe. Arterial blood pressure was measured continuously through a catheter (PE-50) in the femoral artery (Uniflow external pressure transducer, Baxter, Santa Ana, CA, U.S.A.). Heart rate was assessed from the instantaneous pressure signal. To keep the arterial catheter patent it was continuously perfused with physiological saline $(3 \mathrm{ml} / \mathrm{h})$ via the Uniflow system; no heparin was used. To maintain systemic arterial $\mathrm{pH}, \mathrm{pCO}_{2}$, and $\mathrm{pO}_{2}$ at normal values, the rabbits were ventilated throughout the experiments. Ventilation with a positive end-expiratory pressure of $2 \mathrm{~cm} \mathrm{H} \mathrm{H}_{2} \mathrm{O}$ was performed through a tracheal tube ( 3.5 or $4.5 \mathrm{~mm}$ i.d., Mallinckrodt $\mathrm{GmbH}$, Hennef, Germany) connected to an infant ventilator (Loosco, model mk 2, Hoek Loos, Amsterdam, The Netherlands). The rabbits were ventilated with a mixture of oxygen $(25 \%)$, nitrogen (74\%) and carbon dioxide (1\%). Respiration frequency was $60 /$ minute and, depending on the weight of the rabbit, tidal volume was between 11 and $17 \mathrm{ml}$. Blood gas and $\mathrm{pH}$ values were measured in blood collected from a central ear artery, using an acid-base analyzer ( $\mathrm{ABL} 3$, Radiometer, Copenhagen, Denmark). Mean values ( \pm s.d.) were: $\mathrm{pH} 7.42 \pm 0.07, \mathrm{pCO}_{2} 41 \pm 4 \mathrm{mmHg}$, and $\mathrm{pO}_{2} 102 \pm 14 \mathrm{mmHg}$.

Through a small abdominal midline incision, a segment of the distal ileum was exteriorized. The mesentery was carefully spread over a siliconized glass plate mounted in an electrically heated microscope table $\left(37^{\circ} \mathrm{C}\right)$. The preparation was continuously superfused with buffered Tyrode's solution $\left(37^{\circ} \mathrm{C} ; \mathrm{pH} 7.35-7.40\right)$. The bowels were kept moist with overlying wet gauze. Mesenteric venules were visualized with a Leitz intravital microscope, adapted for telescopic imaging (Slaaf et al., 1982), and a Leitz water-immersion objective (SW25, numerical aperture 0.60). Transillumination was performed with a tungsten lamp. Images were recorded on videotape (Sony, Betamax) through a CCD camera (Hamamatsu, C2400). Final magnification at the front plane of the camera was $\times 52$.

Venular diameters (range: 17-32 $\mu \mathrm{m}$ ) were measured off-line with an image shearing device (Intaglietta and Tompkins, 1973), while red blood cell (RBC) velocity was determined on-line using a prism grating system with the slit covering the whole vessel width (Slaaf et al., 1981). To obtain actual mean RBC velocities, the 
measured velocity values were divided by a conversion factor of 1.1 (Slaaf et al. 1986; Tangelder et al., 1986). Reduced velocity U (mean RBC velocity/vessel diameter), which is a measure of wall shear rate, was calculated from these parameters.

Leukocytes were considered to be rolling if they could be seen moving along the vessel wall by eye, either at a rather constant velocity or saltating at a significantly lower rate than the blood was flowing. The ratio of saltating to rolling cells was about $1: 20$; this ratio was not affected by the various test solutions. Therefore, leukocyte rolling was quantitated off-line by counting in duplicate the number of cells that were rolling and saltating along a predefined segment of the venule during a period of $100-120 \mathrm{~s}$. The length of the segment was approximately similar to the diameter of the vessel. The level of leukocyte rolling was expressed as the number of rolling cells passing per minute. Leukocytes were considered to be adherent when they remained stationary for at least $30 \mathrm{~s}$. Adherence of leukocytes was determined in a $100 \mu \mathrm{m}$ segment of the venule. The mean number present at any instant was assessed over a period of 2 minutes. To assure that all rolling leukocytes could be distinguished, the midplane of a venule was kept in focus in all experiments (oude Egbrink et al., 1992).

To allow adequate detection of changes in leukocyte rolling induced by the agents under study, only venules were selected with a baseline level between 10 and 50 rolling leukocytes per minute. Hardly any vessel had to be excluded.

\section{Experimental protocol}

After selection of a mesenteric module, the remaining part of the exposed tissue was covered with clear plastic wrap to prevent quick leakage of the superfusion fluid to the surrounding tissue and the gauze covering the bowels. Subsequently, the tissue was allowed to stabilize for 30 minutes. Video recordings of at least 2 minutes were made per vessel to assess its diameter, and baseline leukocyte rolling and adhesion levels; baseline RBC velocity was measured on-line. Subsequently, superfusion with normal Tyrode's solution was stopped and the test solution (see below) or the vehicle dissolved in Tyrode's solution was superfused for a period of 30 minutes, after which FMLP was added to the test solution for 15 minutes. When FMLP superfusion was stopped, the test solution was superfused for another 30 minutes. All fluids were superfused at a rate of $150-250 \mathrm{ml} / \mathrm{h}$. Temperature of the superfusion fluid was kept at $37^{\circ} \mathrm{C}$. During each step of the experiments video recordings were made and RBC velocity measurements were performed.

Five groups were used in this study: (1) a vehicle group ( $n=7$ rabbits), (2) a $P G_{1}$ group ( $n=5)$, (3) a $\mathrm{PGE}_{2}$ group $(n=6),(4)$ a group receiving the stable prostacyclin $\left(\mathrm{PGI}_{2}\right)$ analogue lloprost $(n=5)$, and $(5)$ a group receiving a combination of $P G E_{1}$, $\mathrm{PGE}_{2}$, and lloprost (referred to as cocktail group; $n=6$ ). PGE (Sigma Chemical, St.

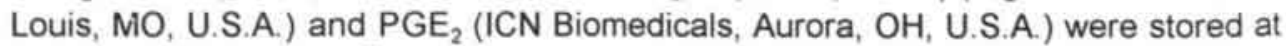
a concentration of $10^{-2} \mathrm{M}$ in absolute ethanol at $-20^{\circ} \mathrm{C}$ until diluted in Tyrode's solution to a final concentration of $10^{-6} \mathrm{M}$. lloprost (kindly provided by Schering AG, Berlin, Germany) was diluted in Tyrode's solution to a final concentration of $10^{-7} \mathrm{M}$. 
The tenfold lower concentration of $10^{-7} \mathrm{M}$ was used because lloprost is known to be 10 times more potent than prostacyclin as an inhibitor of, among others, platelet aggregation (Fisher et al., 1987). A concentration of $10^{-6}-10^{-7} \mathrm{M}$ appears to be biologically relevant. Estimations of human systemic plasma $\mathrm{PGI}_{2}$ concentrations range form approximately $3 \times 10^{-12} \mathrm{M}$ to $0.4 \times 10^{-7} \mathrm{M}$ (Masotti et al., 1981; Coker et al., 1982; Ritter et al., 1983; FitzGerald et al., 1987). When endothelial cells are stimulated $\mathrm{PGI}_{2}$ production may increase several fold (Revtyak et al., 1987). In the vehicle group absolute ethanol was added to the Tyrode's solution $(0.01 \%)$. This ethanol concentration is not expected to influence leukocyte behavior (MacGregor et al., 1988). N-Formyl-methionyl-leucyl-phenylalanine (FMLP; Sigma Chemical, St. Louis, MO, U.S.A.) was dissolved in dimethyl sulfoxide (DMSO) at a concentration of $10 \mathrm{mg} / \mathrm{ml}$ and further diluted to $10^{-6} \mathrm{M}$ in Tyrode's solution, containing PG(s) or vehicle. All solutions were freshly prepared on each experimental day.

\section{Systemic leukocyte counts}

Systemic leukocyte counts were performed at the beginning of the stabilization period, at the end of the FMLP administration, and at the end of each experiment. To this purpose $20 \mu \mathrm{l}$ blood was collected from a central ear artery and added to Türks solution ( $0.2 \mathrm{mg}$ gentian violet in $1 \mathrm{ml}$ glacial acetic acid, $6.25 \%$ vol/vol) in a $1: 10$ dilution; leukocytes were counted and differentiated as polymorphonuclear (PMN) or monomorphonuclear (MMN) in a counting chamber (Clay Adams, Parsippany, N.J., U.S.A.). Of these two leukocyte subclasses the PMNs are the ones that are rolling in rabbit mesenteric venules (Tangelder et al., 1995).

\section{Data analysis and statistics}

Because of their nonsymmetrical distribution, data are presented as medians with interquartile ranges (i.e., the spread from 25 th to 75 th percentile). The data shown in the figures represent results obtained during the last 5-10 minutes of each experimental period. To enable comparisons between groups, some data are presented normalized to their baseline values. Paired data groups were compared using the Wilcoxon signed-rank test. Differences between two independent data groups were tested with the Mann-Whitney $U$ test; to compare more than two independent data groups the Kruskal-Wallis test was used. In all tests, the level of significance was set at 0.05 .

\subsection{Results}

\section{Effect of prostaglandins on leukocyte rolling}

Before superfusion of the PG(s) or the vehicle, the median baseline number of rolling leukocytes per minute in the selected venules was $33(n=64$; interquartile range: $25-38 / \mathrm{min}$ ). No difference in baseline levels of rolling leukocytes could be detected between the groups receiving vehicle $(n=18$; median $34 / \mathrm{min}), \mathrm{PGE}_{1}(n=13$; $30 / \mathrm{min}), \mathrm{PGE}_{2}(n=18 ; 36 / \mathrm{min}), \mathrm{PGI}_{2}(n=16 ; 34 / \mathrm{min})$, or the cocktail $(n=15 ; 33 / \mathrm{min})$. The effect of local superfusion with PGs or vehicle on the level of leukocyte rolling 


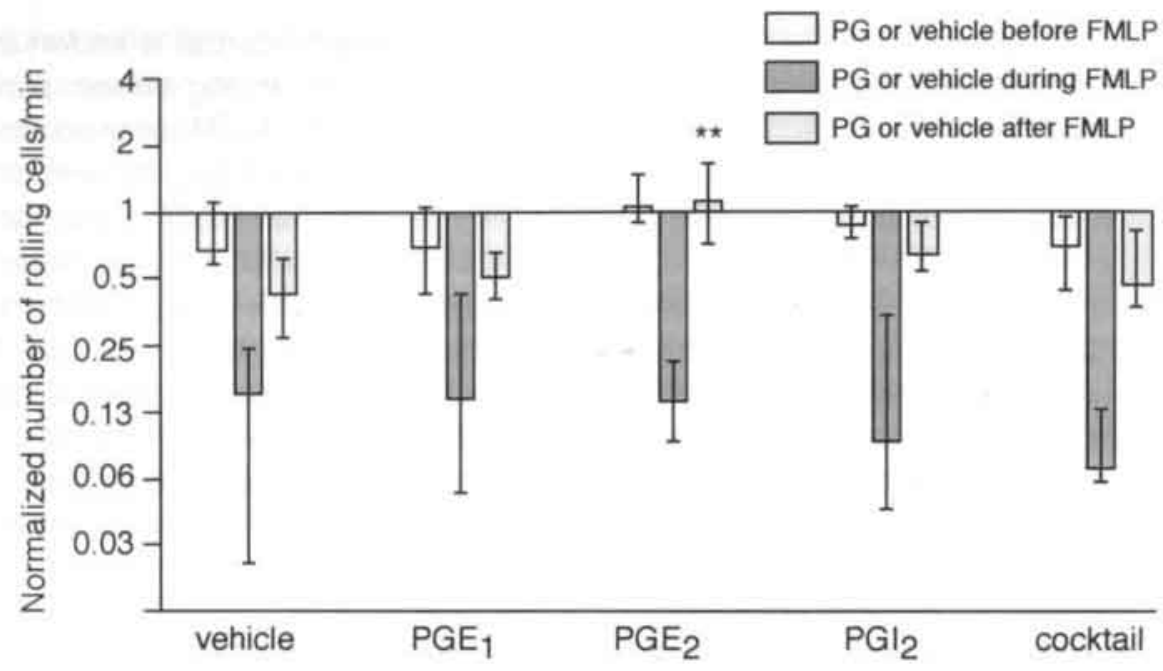

Figure 8.1. Effect of superfusion with vehicle, $P G E_{1}, P G E_{2}, P G_{2}$, or a cocktail of these prostaglandins on the level of leukocyte rolling in rabbit mesenteric venules before, during, and after adding FMLP. Medians and interquartile ranges are shown. Data are normalized to baseline values (1-line).

$* p \leq 0.01$ as compared to the vehicle

before, during and after adding FMLP is presented in Figure 8.1. The figure presents results obtained during the last 5-10 minutes of each experimental period, but no differences were found with the results obtained within the first 3 minutes of each period. In the vehicle group leukocyte rolling decreased in time. As compared to the vehicle none of the PGs influenced the level of leukocyte rolling significantly. After administration of $P G E_{2}$ the level of leukocyte rolling tended to be higher $(p=0.15)$ than in the vehicle group. A higher concentration of lloprost $10^{-5} \mathrm{M}$ also had no effect on an already stabilized level of leukocyte rolling (data not shown). When FMLP was added to the superfusion fluid the overall median level of leukocyte rolling decreased significantly from 23 to 3 rolling leukocytes per minute; no differences between the groups were found. When superfusion with FMLP was stopped, the level of leukocyte rolling returned to the baseline value only in the $\mathrm{PGE}_{2}$ group. As a result, the level of leukocyte rolling was significantly higher in this group than in the vehicle group.

\section{Effect of prostaglandins on leukocyte adhesion}

Figure 8.2 shows the changes in leukocyte adhesion in the course of the experiments. The median baseline number of adherent leukocytes was $1 / 100 \mu \mathrm{m}$ (interquartile range: $0-2 / 100 \mu \mathrm{m}$ ), with no differences between the groups. Superfusion with vehicle or PGs without FMLP did not influence the number of adherent leukocytes. Addition of FMLP to the superfusate induced a significant increase in leukocyte adhesion in all groups; only in the PGE $\mathrm{P}_{2}$ treated animals this effect was significantly greater than in the ones receiving vehicle. The effect of 


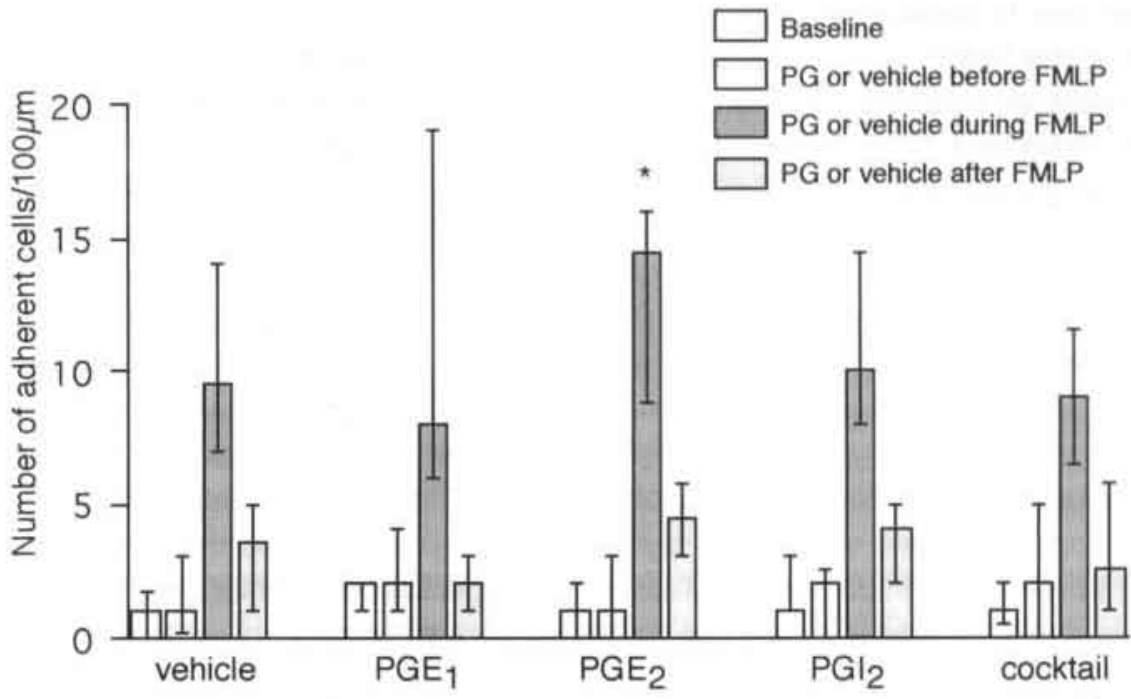

Figure 8.2. Effect of superfusion with vehicle, $P G E_{1}, P G E_{2}, P G I_{2}$, or a cocktail of these prostaglandins on leukocyte adhesion in rabbit mesenteric venules before, during, and after adding FMLP. Medians and interquartile ranges are shown.

- $p \leq 0.05$ as compared to the vehicle

FMLP superfusion occurred within seconds and did not change during the whole superfusion period. After FMLP had been washed out, the number of adherent cells almost returned to baseline values, indicating that the effect of FMLP is reversible.

\section{Fluid dynamics}

The fluid dynamic parameters, as measured in the selected venules before administration of the PGs, are presented in Table 8.1. No differences in baseline diameter existed between the groups. RBC velocity was higher in the cocktail group as compared to the vehicle group throughout the experimental periods; the relative changes, however, were not significantly different. The baseline values of reduced velocity $\mathrm{U}$ were not different between the groups. Reduced velocity was higher following superfusion with the cocktail as compared to the vehicle group, but again the relative changes were not significantly different. The fact that no major differences between the groups were found indicates that the changes in leukocyte rolling and adhesion observed following administration of prostaglandins and/or FMLP cannot be explained by hemodynamic changes. This is supported by the observation that no correlations were found between flow and the (changes in) level of leukocyte rolling or adhesion.

Baseline mean arterial pressure and heart rate were $75 \mathrm{mmHg}$ (interquartile range: $70-85 \mathrm{mmHg}$ ) and 116 beats per minute $(112-120 \mathrm{bpm})$, respectively, with no differences between the groups. During local administration of FMLP mean arterial pressure fluctuated temporarily in most groups and was in all groups except the cocktail group found to be increased at the end of the 15 minute superfusion period; 
heart rate fluctuated as well and was found to be in these groups decreased at the end of the FMLP administration. On the contrary, in the cocktail group mean arterial pressure was decreased and heart rate increased following superfusion with FMLP (63 $\mathrm{mmHg}$ and $122 \mathrm{bpm}$ in the cocktail group versus $88 \mathrm{mmHg}$ and $103 \mathrm{bpm}$ in the vehicle group; both $p \leq 0.01$ ).

\section{Systemic leukocyte counts}

Before the experiments started, in all rabbits both the systemic number of leukocytes (median $6.6 \times 10^{6} / \mathrm{ml}$; interquartile range $4.8-7.9 \times 10^{6} / \mathrm{ml}$ ) and the percentage of polymorphonuclear cells $(36 \% ; 30-41 \%)$ were within normal ranges (Kozma et al., 1974). Following superfusion with FMLP, the systemic number of leukocytes decreased significantly in all groups; at the end of the experiments the number had returned to baseline values. No significant differences existed between the groups. The percentage of polymorphonuclear cells increased slightly (with an average of $12 \%$ ), but significantly during the experiments in all groups; the differences between the groups were not significant.

Table 8.1. Baseline fluid dynamic parameters in venules of the various groups before superfusion of the rabbit mesentery with vehicle or prostaglandins. Median values with interquartile ranges in parentheses are presented.

\begin{tabular}{lccccc}
\hline & vehicle & $\mathrm{PGE}_{1}$ & $\mathrm{PGE}_{2}$ & $\mathrm{PGI}_{2}$ & cocktail \\
\hline Diameter, $\mu \mathrm{m}$ & 22 & 24 & 24 & 23 & 25 \\
& $(19-25)$ & $(22-26)$ & $(21-26)$ & $(21-25)$ & $(21-26)$ \\
RBC vel, $\mathrm{mm} / \mathrm{s}$ & 1.2 & 1.1 & 1.1 & 1.6 & $1.4^{*}$ \\
& $(0.8-1.6)$ & $(0.9-1.1)$ & $(0.7-1.3)$ & $(1.1-2.1)$ & $(1.2-2.2)$ \\
$\mathrm{U}, \mathrm{s}^{-1}$ & 50 & 44 & 38 & 67 & 67 \\
& $(42-66)$ & $(38-50)$ & $(29-62)$ & $(43-88)$ & $(53-91)$ \\
\hline
\end{tabular}

$R B C$ vel indicates mean red blood cell velocity and $U$, reduced velocity (mean red blood cell velocity per diameter). " $p \leq 0.05$ versus vehicle

\subsection{Discussion}

In the present study we show that the prostaglandins $E_{1}, E_{2}$, and $I_{2}$, and also a combination of the three, do not influence the rolling of leukocytes along the endothelium of postcapillary venules in the exteriorized rabbit mesentery. Leukocyte adhesion induced by the chemo-attractant FMLP was highest in the presence of $\mathrm{PGE}_{2}$, whereas in all groups a similar decrease in the level of leukocyte rolling was found following administration of FMLP. When FMLP superfusion was stopped the number of adherent cells almost returned to baseline, whereas the level of leukocyte rolling increased. Only in the presence of $\mathrm{PGE}_{2}$ the number of rolling leukocytes returned to baseline levels, resulting in a significantly higher level of leukocyte rolling after FMLP administration in the $\mathrm{PGE}_{2}$ treated rabbits as compared to the vehicle. 
The fact that the employed PGs did not reduce an already stabilized level of leukocyte rolling or FMLP-induced leukocyte adhesion suggests that a possible beneficial effect of PGs on leukocyte function in various vascular diseases is probably not based on inhibition of the two initial steps leading to diapedesis and migration of leukocytes. The reported beneficial effects of PGs in vascular diseases in vivo may, as far as they are related to leukocytes, be based on the inhibition of the production of superoxide anions and degradative enzymes by PMNs after these cells have infiltrated into the tissue. These inhibiting effects of PGs have been demonstrated in in vitro and ex vivo studies (Fantone et al., 1984; Kanamori et al., 1997).

Our present findings seem to be in contrast to those of one of the few other in vivo studies in which the effect of PGs on leukocyte-endothelium interactions was studied

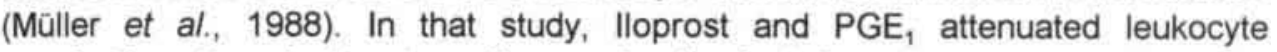
margination (i.e., leukocyte rolling and adhesion) in rat mesentery. However, leukocyte-endothelium interactions in that study were induced by electrical stimulation of the vessel wall, which may have been severely damaged, causing endothelial cell dysfunction. Exogenously administered PGs may have been protective under these circumstances.

Instead of an inhibiting effect on leukocyte-vessel wall interactions, $\mathrm{PGE}_{2}$ appeared to have an extra stimulating effect on FMLP-induced leukocyte adhesion. The higher level of leukocyte adhesion observed in the presence of $\mathrm{PGE}_{2}$ may eventually lead to increased leukocyte infiltration into the tissue, as found in rabbit cervices after vaginal application of this PG (El Maradny et al., 1996). Comparable results were obtained ex vivo, showing that the adherence of human lymphocytes to virus-infected cells was increased after ingestion of $\mathrm{PGE}_{2}$ (Dore-Duffy et al., 1984). The observed increased adhesion may have been due to $\mathrm{PGE}_{2}$-stimulated expression of the intercellular adhesion molecule-1 (ICAM-1) which has been observed in cultured umbilical vein endothelial cells (Winkler et al., 1997). Unfortunately, in these studies no comparison with other PGs has been made. A second, and plausible explanation for the observed $\mathrm{PGE}_{2}$ effect is that the larger number of adherent cells in the $\mathrm{PGE}_{2}$ group was the result of the larger number of rolling cells in this group before administration of FMLP (38/min as compared to 21 , 26,30 , and $23 / \mathrm{min}$ in the vehicle, PGE ${ }_{1}$, lloprost, and cocktail groups, respectively). because a close relationship has been demonstrated between initial leukocyte rolling and subsequent chemoattractant-induced firm adhesion (Lindbom et al., 1992). The fact that the ratio of the level of leukocyte rolling before administration of FMLP and the number of adherent leukocytes during superfusion with FMLP is independent of the presence of PGs, supports this hypothesis.

The effect of FMLP on leukocyte adhesion appeared to be almost completely reversible, with no influence of the PGs. After FMLP administration was stopped, the level of leukocyte rolling increased, but, except for the $P G E_{2}$ group, did not return to baseline. Because FMLP induced fluctuations in mean arterial pressure and heart rate and a decrease in the systemic number of leukocytes, it is plausible that in our experiments FMLP did enter the systemic circulation. This implicates that circulating 
leukocytes may have been activated by FMLP and, subsequently, may have shed their L-selectin (Neeley et al., 1993). This could explain why the level of leukocyte rolling did not return to baseline in most groups. $\mathrm{PGE}_{2}$ is known to inhibit L-selectin shedding as induced by platelet-activating factor (PAF) (Berends et al., 1997). It is possible that $\mathrm{PGE}_{2}$ inhibits FMLP-induced L-selectin shedding as well, which may have contributed to the complete return of leukocyte rolling to the baseline level during the administration of this PG.

One could argue that the superfused PGs might not have entered the lumen of the venules under investigation. Our first argument that they do is that our data show that $\mathrm{PGE}_{2}$ does affect leukocyte rolling, indicating that it has reached the venular endothelium. Secondly, with fluorescence microscopy we were able to show that superfusion of the rabbit mesentery with the fluorescent dye acridine red results in the appearance of fluorescence in the lumen of venules within a few seconds (S.C.T., unpublished observation). Likewise, FMLP influenced leukocyteendothelium interactions within seconds following topical application. Because the molecular weights of the PGs are between those of acridine red and FMLP (acridine red: 275.7, PGE $: 352.5, \mathrm{PGE}_{1}: 354.5$, lloprost: 360.5 and FMLP: 437.6), and PGE is able to reach the endothelium (see above), it is very likely that the other superfused PGs entered the lumen of the venules, too. The continuous administration of the test solutions precludes complete disappearance from the tissue due to metabolization of the prostaglandins before they reach the vessels.

One may argue that the absence of a significant influence of the employed PGs on leukocyte rolling and FMLP-induced adhesion may be due to an absence of PGreceptors or a low affinity of such receptors in rabbit mesenteric microvessels, which would also explain our finding that the PGs used had no effect on vascular diameters. Several other studies, however, showed that in the same tissue PGs do play a role in the interactions between vessel wall and blood platelets (Bergqvist and Arfors, 1976; Arfors and Bergqvist, 1978; oude Egbrink et al., 1993): inhibition or administration of PGs significantly influences hemostatic plug formation and thromboembolism. An important point in this repect is that in all these studies the PG-effects differ in arterioles and venules, which clearly indicates that these effects cannot be explained solely by an influence of PGs on blood platelets: the vessel walls have to be involved in the changes induced. Hence, these studies show that PGs are produced in rabbit mesenteric microvessels and that they influence vascular functions, strongly suggesting the presence of functional PG-receptors in microvessels in the rabbit mesentery.

It is not surprising that the applied PGs did not have any effect on the diameter of the venules under study, although $\mathrm{PGE}_{2}$ and $\mathrm{PGI}_{2}$ are known to be potent vasodilator agents (Kaley et al., 1985). This is due to the fact that microvessels in the rabbit mesentery have a low level of vasoactivity: local fluid dynamic conditions do not change after superfusion with such vasodilators as adenosine or a nitric oxide donor, or with such vasoconstrictors as a nitric oxide synthesis inhibitor, noradrenaline or potassium chloride (Broeders et al., 1998). Furthermore, no alterations in diameter and blood flow velocity were found in arterioles and venules 
of the rabbit mesentery after 10 minutes of tissue hypoxia by occlusion of the superior mesenteric artery (Olofsson et al., 1994). In addition, local intraluminal administration of a corresponding concentration of lloprost did not alter diameter and red blood cell velocity either, indicating that the absence of a vasodilating effect is not due to the route of administration (S.C.T. unpublished results). Even infusion of lloprost in a concentration that was 100 to 1000 times higher did not alter local diameter, but decreased mean arterial pressure, demonstrating that lloprost administered in this way has systemic dilating effects. Fluctuations in blood pressure and heart rate during administration of FMLP have been described before (Saito et al., 1983; Jonsson et al., 1997) and are thought to be caused by a direct effect on the vascular tone or an indirect effect through the release of products by FMLPstimulated granulocytes.

The present findings indicate that the PGs under investigation are probably not the endogenous agents responsible for the reduction of leukocyte rolling as observed in mesenteric venules during a thromboembolic process (oude Egbrink et al., 1992). Even a combination of these PGs (the cocktail) did not influence leukocyte rolling, suggesting that these PGs, which can be released by microvascular endothelial cells simultaneously (Gerritsen and Cheli, 1983; Linssen et al., 1993), do not act synergistically in inhibiting leukocyte rolling.

In conclusion, $P \mathrm{PE}_{1}, \mathrm{PGE}_{2}, \mathrm{PGI}_{2}$, or a cocktail of these PGs do not affect a stabilized level of leukocyte rolling or FMLP-induced leukocyte adhesion. This suggests that leukocyte related beneficial effects of PGs in various vascular diseases are due to other effects than inhibition of leukocyte rolling and adhesion. 



\section{TUMOR ANGIOGENESIS FACTORS REDUCE LEUKOCYTE ADHESION IN VIVO}

Submitted as: S.C. Tromp, M.G.A. oude Egbrink, J.S. van Velzen, D.W. Slaaf, H.F.P. Hillen, G.J. Tangelder, R.S. Reneman, and A.W. Griffioen. Tumor angiogenesis factors reduce leukocyte adhesion in vivo.

\section{Abstract}

Leukocyte-endothelium interactions are diminished in tumors. It is reported here that this phenomenon is due to endothelial cell exposure to growth factors involved in tumor angiogenesis. To demonstrate this, independently from tumor cell associated processes, we used a tumor free model. Slow releasing pellets were loaded with either basic fibroblast growth factor (bFGF), vascular endothelial cell growth factor (VEGF), or vehicle alone and placed in the scrotum of mice. After 3 days, a single intrascrotal injection of $1 \mu \mathrm{g} / \mathrm{kg} \mathrm{IL}-1 \beta$ was given 4 hours before vessels of the cremaster muscle were investigated for leukocyte rolling and adhesion by means of intravital microscopy. Exposure of normal tissue to either bFGF or VEGF resulted in markedly decreased levels of cytokine induced leukocyte adhesion. Suppression of leukocyte rolling was not observed. Instead a moderate enhancement of rolling by VEGF was found. The observed differences could not be explained by differences in fluid dynamic parameters or systemic leukocyte counts. In conclusion, evidence is presented that, in vivo, proangiogenic factors significantly reduce leukocyte adhesion, the final step preceding leukocyte infiltration. This observation may explain why tumors escape from immune surveillance. 


\subsection{Introduction}

Leukocyte infiltration in tumors has been reported to be associated with improved prognosis (Underwood, 1974; Dvorak and Dvorak, 1982). Furthermore, inflammation (Arca et al., 1996) and immunotherapy (Chang and Shu, 1996) have been shown to be effective in tumor regression. For infiltration from the blood stream into surrounding tissue, leukocytes have to interact with microvascular endothelium. The first interactive step is the slow rolling of leukocytes along the endothelial lining, which subsequently may result in firm adhesion, diapedesis and emigration into the surrounding tissue (von Andrian et al., 1992; Springer, 1994). Since leukocyte infiltration may be detrimental to a tumor, escape mechanisms may have evolved to avoid the infiltration of leukocytes into the tumor. Prevention of leukocyte rolling along or adhesion to endothelium may be such a mechanism. Indeed, in several studies leukocyte-endothelium interactions, i.e., leukocyte rolling and adhesion, were found to be reduced in tumor microvessels (Wu et al., 1992; Dellian et al., 1996; Borgström et al., 1997). The mechanism behind this reduction has not yet been fully elucidated, although several hypotheses exist. A low delivery of leukocytes to the angiogenic microvessels in tumors has been described (Dellian et al., 1996; Jain et al., 1996) as well as a diminished expression of adhesion molecules involved in leukocyte-endothelium interactions in these vessels (Kuzu ot al., 1993; Piali et al., 1995; Griffioen, 1997).

Tumor outgrowth and metastasis are dependent on the formation of new vasculature. To achieve this, tumor cells produce high levels of angiogenic factors, such as basic fibroblast growth factor (bFGF) and vascular endothelial cell growth factor (VEGF). In vitro these factors have been shown to reduce the expression of endothelial adhesion molecules such as intercellular adhesion molecule-1 (ICAM-1) (Kitayama et al., 1994; Griffioen et al., 1996b; Melder et al., 1996), ICAM-2, and CD34 (Griffioen et al., 1996a; Hellwig et al., 1997). In addition, the cytokine induced expression of vascular cell adhesion molecule-1 (VCAM-1), and E-selectin on cultured endothelial cells is markedly inhibited by these pro-angiogenic factors (Griffioen et al., 1996b). To investigate the regulatory function of angiogenic factors on leukocyte-endothelium interactions in vivo. we used a tumor free model to avoid complex tumor associated processes. Pellets slowly releasing angiogenic factors were placed in the mouse cremaster muscle and after three days cytokine induced leukocyte-endothelium interactions were analyzed. We provide the first evidence that both bFGF and VEGF reduce leukocyte adhesion in vivo.

\subsection{Materials and methods}

\section{Preparation of growth factor pellets}

Heparin-alginate pellets were prepared as previously described (Edelman et al., 1993: Lopez et al., 1997) with slight modifications. In short, heparin-Sepharose beads (Pharmacia, Roosendaal, the Netherlands) were mixed with sodium alginate (Orffa, Drammen, Norway). The slurry was then allowed to polymerize in a matrix. Pellets were incubated in $\mathrm{CaCl}_{2}$ for hardening. The pellets were then sterilized in $70 \%$ ethanol, 
washed with sterile water and stored in physiological saline containing $1 \mathrm{mM} \mathrm{CaCl} \mathrm{m}_{2}$ at $4^{\circ} \mathrm{C}$. Each pellet had a volume of $8 \mu \mathrm{l}$. For loading with growth factors, pellets were incubated for 2 days at $4^{\circ} \mathrm{C}$ in $300 \mathrm{ng}$ of human recombinant basic fibroblast growth factor (bFGF) or human recombinant vascular endothelial cell growth factor (VEGF; both from Pepro Tech, London, U.K.) in a volume of $20 \mu \mathrm{l}$. In vivo activity of these pellets in mice was demonstrated by the formation of new vasculature in the dorsal skinfold chamber after 4 to 5 days (Griffioen; unpublished observations).

\section{Animals and experimental protocol}

The experiments were approved by the local ethical committee on the use of laboratory animals. They were performed on male Swiss mice (Iffa credo, Someren, the Netherlands), with body weights varying from 32 to $39 \mathrm{~g}$.

One pellet, filled with either $300 \mathrm{ng}$ bFGF ( 6 mice), $300 \mathrm{ng}$ VEGF ( 6 mice), or phosphate buffered saline (PBS; 4 mice) as control, was placed in the scrotum of a halothane $(1.5-2.0 \%$ in $80 \%$ oxygen) anesthetized mouse on day 0 . On day 1 and 2 extra bolus injections of bFGF ( $30 \mathrm{ng}$ ). VEGF ( $30 \mathrm{ng}$ ) or PBS in a volume of about 0.2 $\mathrm{ml}$ were given intrascrotally under halothane sedation ( $4 \%$ halothane in $50 \%$ oxygen for 1 minute). Each cremaster muscle was exposed to either bFGF, VEGF or PBS for 3 days, because maximal adhesion molecule regulation was observed after this period in vitro. In all animals murine recombinant interleukin- $1 \beta$ (IL-1 $\beta ; 1 \mu \mathrm{g} / \mathrm{kg}$ in $0.2 \mathrm{ml}$ isotonic saline) was injected intrascrotally under sedation on day $3,2 \frac{1}{2}$ hours before preparation of the cremaster muscle was started. This was performed because leukocyte adhesion is hardly present in the absence of cytokines, which would preclude the assessment of a reduction in leukocyte adhesion, if any.

\section{Intravital microscopy experiments}

Anesthesia was induced with an intraperitoneal injection of sodium pentobarbital (60 $\mathrm{mg} / \mathrm{kg}$ body weight in a volume of $10 \mathrm{ml} / \mathrm{kg}$ ), given under a short halothane sedation (see above), and was maintained by continuous intravenous infusion of sodium pentobarbital $(60 \mathrm{mg} / \mathrm{kg} / \mathrm{h}$ ) through a catheter (pulled PE-10) in the right jugular vein. Arterial blood pressure was measured continuously through a catheter in the carotid artery (pulled PE-10; Uniflow external pressure transducer, Baxter, Santa Ana, CA, U.S.A.). To keep the arterial catheter patent it was continuously perfused with physiological saline $(0.5-1 \mathrm{ml} / \mathrm{h}$; Uniflow system). Mean arterial pressure was continuously recorded on a computer harddisk using a data acquisition system. Throughout the experiments body temperature was kept at $37^{\circ} \mathrm{C}$ by means of an infrared heating lamp controlled by a thermo-analyzer system connected to a subcutaneously placed temperature probe.

The cremaster muscle was prepared for intravital microscopy as described previously (Baez, 1973). In short, a longitudinal incision of the skin and fascia was made in the midline of the scrotum. The right testis was exposed and the connective tissue carefully stripped away. A ventral incision was made along the entire length of the cremaster muscle before it was unwrapped from the testis. The vessels connecting the epididymis to the cremaster were cauterized and the testis and epididymis were gently pushed 
back into the peritoneal cavity. The muscle was spread with the help of sutures at its periphery. The tissue was continuously superfused with $\mathrm{Krebs}$ solution $\left(34-35^{\circ} \mathrm{C} ; \mathrm{pH}\right.$ 7.35-7.40) bubbled with a mixture of $\mathrm{O}_{2}(95 \%)$ and $\mathrm{CO}_{2}(5 \%)$.

At the end of the preparation, the muscle was covered with clear plastic wrap to minimize tissue dehydration, and it was allowed to stabilize for 30 minutes. After stabilization, 4 hours after IL-1 1 injection venules were visualized using a Leitz intravital microscope, adapted for telescopic imaging (Slaaf et al., 1982) and equipped with a water-immersion objective (Leitz SW25, numerical aperture 0.60). Transillumination was performed with a tungsten lamp. Images were recorded on videotape (Sony, Betamax) through a CCD camera (Hamamatsu, C2400). Final optical magnification at the front plane of the camera was $\times 52$. On the average we observed 10 venules per mouse; in 10 mice an average of 3 arterioles was additionally observed.

\section{Experimental parameters}

Venular diameters (range: 18-42 $\mu \mathrm{m}$ ) were measured off-line with an image shearing device (Intaglietta and Tompkins, 1973), while red blood cell (RBC) velocity was determined on-line using a prism grating system with the slit covering the whole vessel width (Slaaf et al., 1981). To obtain actual mean RBC velocities, the measured velocity values were divided by a conversion factor of 1.1 (Slaaf et al., 1986; Tangelder et al., 1986). Reduced velocity $U$ (mean RBC velocity/vessel diameter), which is a measure of wall shear rate, was calculated from these parameters.

Leukocytes were considered to be rolling if they could be seen moving along the vessel wall by eye at a significantly lower rate than the blood was flowing. To assure that all rolling leukocytes could be distinguished, the midplane of a vessel was kept in focus in all experiments (oude Egbrink et al., 1992). Leukocyte rolling was quantified by determining the level of leukocyte rolling as well as the rolling velocity. The level of leukocyte rolling was measured off-line by counting in duplicate the number of cells that rolled through a predefined segment of the vessel during a period of 100 seconds. It was expressed as the number of rolling cells passing per minute. The velocity of the rolling leukocytes was determined off-line by measuring the median time taken by 10 randomly chosen rolling leukocytes to travel a certain distance along the vessel wall. Leukocytes were considered to be adherent when they remained stationary for at least $30 \mathrm{~s}$. The number of adherent leukocytes present at any instant in a $100 \mu \mathrm{m}$ segment of the vessel was assessed and averaged over a period of 2 minutes. This parameter was expressed as number of cells per endothelial surface area. Values of these parameters were randomly checked by a second observer.

The experiments and analyses were performed blindly: the researcher did not know to which group a mouse belonged.

\section{Systemic leukocyte counts}

To perform systemic leukocyte counts, samples of $20 \mu \mathrm{l}$ blood were collected through the arterial catheter during the surgical procedure and in some animals also at the end of the experiment. Each blood sample was administered to Türks solution $(0.2 \mathrm{mg}$ gentian violet in $1 \mathrm{ml}$ glacial acetic acid, $6.25 \% \mathrm{vol} / \mathrm{vol}$ ) in a 1:10 dilution; leukocytes 
were counted and differentiated as polymorphonuclear (PMN) or monomorphonuclear (MMN) in a counting chamber (Clay Adams, Parsippany, N.J., U.S.A.).

\section{Statistics}

Because of their nonsymmetrical distribution, data are presented as medians with interquartile ranges (i.e., the spread from 25 th to 75 th percentile). Differences between two independent data groups were tested with the Mann-Whitney $\mathrm{U}$ test; to compare more than two independent data groups the Kruskal-Wallis test was used. In all tests, the level of significance was set at 0.05 .

\subsection{Results}

Leukocyte adhesion but not rolling is inhibited by both bFGF and VEGF After preparation of the cremaster muscle, leukocyte-endothelium interactions were studied in the PBS, bFGF, and VEGF groups, 4 hours after local IL-1 $\beta$ administration. The most remarkable result was that leukocyte adhesion was significantly $(p \leq 0.01)$ lower than control (i.e., the PBS group) in both the bFGF and the VEGF group (Figure 9.1; control: 1068 cells $/ \mathrm{mm}^{2}$ surface area, bFGF: $658 / \mathrm{mm}^{2}$, and VEGF: $663 / \mathrm{mm}^{2}$ ).

The level of leukocyte rolling was significantly higher in the presence of VEGF (10/min), while bFGF (7/min) had no effect as compared to control (6 cells/min; see Figure 9.2). Leukocyte rolling velocity was slightly higher in the presence of bFGF (median: $5 \mu \mathrm{m} / \mathrm{s}$ versus $4 \mu \mathrm{m} / \mathrm{s}$ in the control mice), while VEGF had no effect ( $4 \mu \mathrm{m} / \mathrm{s}$; data not shown).

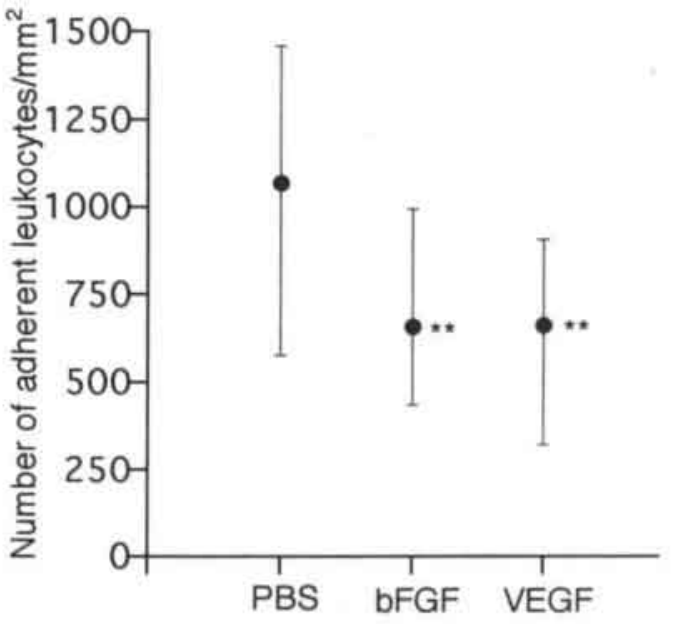

Figure 9.1. Effects of three days pretreatment with bFGF or VEGF on leukocyte adhesion in venules as evoked by local administration of IL-1 $1 \beta$ four hours prior to observation. Medians and interquartile ranges are presented. "* $\mathrm{p} \leq 0.01$ versus control (PBS) 


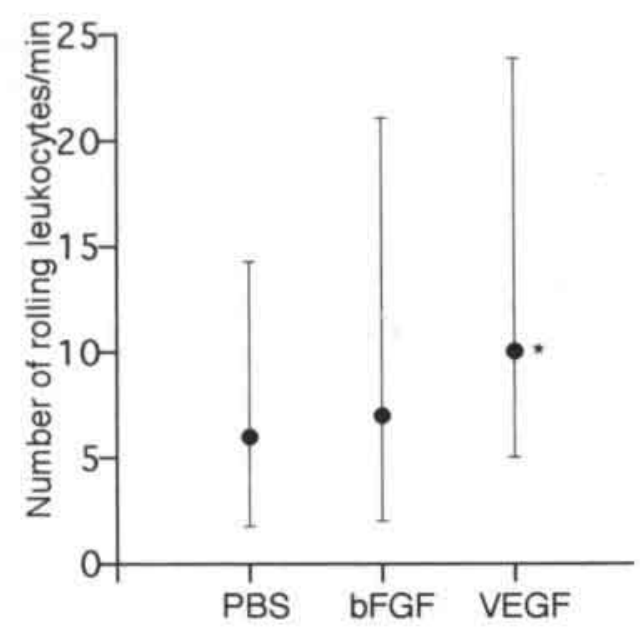

Figure 9.2. Effects of three days pretreatment with bFGF or VEGF on the level of leukocyte rolling in venules after local administration of $\mathrm{IL}-1 \beta$ four hours prior to observation. Medians and interquartile ranges are presented. $" p \leq 0.05$ versus control (PBS)

Rolling leukocytes were also present in most of the arterioles (in 5 of the 6 arterioles observed in the PBS group, 4/4 in the bFGF group and 17/22 in the VEGF group). The level of leukocyte rolling was clearly decreased in the bFGF (median: $3 / \mathrm{min}$ ) and VEGF (6.5/min) groups as compared to the PBS group $(29 / \mathrm{min})$. Leukocyte rolling velocity was higher in arterioles than in venules: median velocity of 28,44 , and $33 \mu \mathrm{m} / \mathrm{s}$ in the PBS, bFGF, and VEGF group, respectively. Hardly any adherent leukocytes were observed in the arterioles of all groups.

\section{Fluid dynamic parameters}

No major differences in the fluid dynamic parameters could be detected between the various groups (Table 9.1), except for a small difference in diameter between the control and VEGF groups. Therefore, it can be excluded that the differences in leukocyteendothelium interactions between the groups were caused by differences in fluid dynamics.

In all animals mean arterial blood pressure ranged between 80 and $120 \mathrm{mmHg}$, which is in the normal range for anesthetized mice (Desai et al., 1997).

\section{Systemic leukocyte counts}

Median systemic number of leukocytes during the surgical procedure at about 3 hours after IL-1 $\beta$ injection were $4.2 \times 10^{6} / \mathrm{ml}$ blood in the PBS group, $5.4 \times 10^{6} / \mathrm{ml}$ in the bFGF group, and $5.9 \times 10^{6} / \mathrm{ml}$ in the VEGF group. These differences were not statistically significant. In the course of the experiments the systemic number of leukocytes decreased in all animals by about $50 \%$; still no differences between the groups were found, indicating that the differences in leukocyte-endothelium interactions between the groups are not caused by differences in systemic leukocyte counts. The percentage of PMNs was 60,62 , and $63 \%$ in the PBS, bFGF, and VEGF group, respectively; these 
values did not change during the experiments. The high percentage of PMNs may be attributed to systemic effects of the locally injected IL-1B.

\section{Macroscopic observations}

After the pellets with bFGF, VEGF, or PBS had been in the scrotum for 3 days, the scrotum was opened and a number of features were macroscopically observed. In 5 mice the connective tissue around the cremaster muscle was more abundant and less gelatinous than in others. It could, however, easily be separated from the muscle and did not hamper microscopic observation. Three of these mice were treated with bFGF. In 8 mice the vessels in the connective tissue surrounding the cremaster muscle and in the muscle itself had a high tendency to bleed easily; 5 of these mice had been treated with VEGF.

Table 9.1. Fluid dynamic parameters in venules of the cremaster muscle of mice treated with PBS, bFGF, or VEGF for 3 days. Data are median values with interquartile ranges in parentheses.

\begin{tabular}{llll}
\hline & PBS & bFGF & VEGF \\
\hline$n$ & 36 & 69 & 58 \\
Diameter $(\mu \mathrm{m})$ & $26(24-28)$ & $28(24-30)$ & $28(24-32)$ \\
RBC vel $(\mathrm{mm} / \mathrm{s})$ & $0.5(0.3-0.7)$ & $0.6(0.3-0.8)$ & $0.5(0.3-0.7)$ \\
$U\left(\mathrm{~s}^{-1}\right)$ & $17(10-30)$ & $20(12-28)$ & $16(11-25)$ \\
\hline
\end{tabular}

$n=$ number of venules, $R B C$ vel = mean red blood cell velocity, $U=$ reduced velocity

\subsection{Discussion}

It has been demonstrated previously that in tumor endothelial cells the expression of adhesion molecules, involved in leukocyte-endothelium interactions, is suppressed (Kuzu et al., 1993; Piali et al., 1995; Griffioen, 1997). In addition, the upregulation of endothelial adhesion molecules in response to inflammatory cytokines in tumors was shown to be markedly hampered (Griffioen et al., 1996a). Exposure of the endothelial cells to tumor derived factors appeared to be responsible for these phenomena (Kitayama et al., 1994; Griffioen et al., 1996b). Since this knowledge is exclusively based on studies that were performed in vitro, we developed a model to selectively study the effects of single tumor derived factors on leukocyte-endothelium in vivo. With the use of intravital videomicroscopy we demonstrated a reduction of IL-1 $\beta$ induced leukocyte adhesion by the tumor derived angiogenesis factors bFGF and VEGF in the mouse cremaster muscle. Hence, we provide evidence for a tumor mediated escape from the immune system.

In a number of earlier studies it has been demonstrated that leukocyte-endothelium interactions are diminished in tumor vessels in vivo (Wu et al., 1992; Wu et al., 1994; Dellian et al., 1995; Fukumura et al., 1995; Borgström et al., 1997). In these studies, the mechanism behind this low level of interaction was not elucidated. Beside angiogenic products of tumor cells, excreted cytokines but also deviating local interstitial and/or blood pressures may have been involved. The same holds true for a study of Brown and colleagues (1997), which showed increased leukocyte adhesion in the mouse cremaster 
muscle that was superfused with tumor conditioned medium: other than angiogenic factors may have had an effect. The present study is the first in which only the effects of tumor derived angiogenesis factors are investigated in vivo. We demonstrated in a tumor free system that neither fluid dynamic parameters nor the number of circulating leukocytes can account for the observed reduction in leukocyte adhesion by bFGF and VEGF. Similarly, Wu and colleagues found decreased leukocyte-endothelium interactions in normal venules surrounding an implanted tumor compared with normal vessels in the same tissue without a tumor (Wu et al., 1994).

The present study demonstrates for the first time that a local release of either bFGF or VEGF reduces leukocyte adhesion in vivo. One of the possible mechanisms underlying this phenomenon, is the downregulation of endothelial adhesion molecules. We were not able to demonstrate this mechanism in the present model because immunohistochemistry is not sensitive enough to exactly quantify a change in ICAM-1 expression on endothelial cells, and the mouse cremaster muscle is too small to provide a sufficient number of endothelial cells to perform flowcytometric analysis. However, our findings are compatible with earlier findings of studies on in vitro adhesion and on expression of endothelial adhesion molecules (Griffioen et al., 1996a; Griffioen et al., 1996b), which show that endothelial adhesion molecules such as ICAM-1 and VCAM-1 are downregulated in the presence of angiogenic factors resulting in diminished leukocyte adhesion. Our findings seem to be in contrast with an earlier study by Melder et al. (1996), which suggested that bFGF and VEGF have opposite functions with regard to leukocyte adhesion. While bFGF inhibited adhesion both in vitro and in vivo, the effect of VEGF was only investigated in vitro with HUVEC that were cultured without serum. In this setting VEGF promoted adhesion. A stimulating effect of VEGF on leukocyte adhesion was also observed by Detmar and colleagues (1998). However, the experimental approach in their study in which the effects of a systemic overexpression of VEGF in transgenic mice were investigated, is completely different from the situation in tumors where VEGF is produced locally. A recent study, however, is more consistent with our present findings and identifies VEGF as a potent inhibitor of leukocyteendothelium interactions (Scalia et al., 1999).

In our study we also addressed leukocyte rolling and we demonstrated that bFGF did not affect this, while VEGF showed a small, but significant increase. The increased rolling in the VEGF group might be explained by an upregulation of E-selectin by VEGF (Melder et al., 1996). It may also be an epiphenomenon of the reduced leukocyte adhesion, although it is not clear why we do not find this epiphenomenon in the bFGF treated animals. Nonetheless, increased leukocyte rolling is meaningless with respect to leukocyte infiltration, if it is not followed by firm adhesion.

In our cremaster preparations, leukocyte rolling was also observed in arterioles. This phenomenon is absent in mouse cremaster muscles not pretreated with cytokines (Thorlacius et al., 1997 and Tromp et al., unpublished observations), but has been reported before in IL-1 $\beta$ and/or tumor necrosis factor (TNF) $\alpha$ stimulated mouse cremaster muscle (Thorlacius et al., 1997). It is supposed to be a P-selectin mediated event, since it can be abolished by administration of an anti-P-selectin antibody. The reduced number of rolling cells in arterioles after treatment with bFGF or VEGF 
suggests that these angiogenic factors are able to diminish the expression of P-selectin at the endothelial surface in these microvessels.

The observed increased amount of connective tissue in a large number of bFGF treated animals was to be expected, because the primary function of bFGF is to stimulate fibroblasts to proliferate (Sprugel et al., 1987). The relatively high tendency of vessels to bleed easily in the presence of VEGF is probably due to its ability to increase microvascular permeability; VEGF was originally described as vascular permeability factor. In rat cremaster muscle and mouse skin, electron microscopy revealed, among others, leakage of red blood cells from endothelial intercellular gaps after topical VEGF application (Roberts and Palade, 1995).

In conclusion, the mouse cremaster muscle is a practical and suitable model to selectively study the effects of single tumor derived factors on leukocyte-endothelium interactions in a tumor free environment. In this tissue we demonstrated that the tumor derived angiogenesis factors bFGF and VEGF inhibit IL- $1 \beta$ induced leukocyte adhesion, the final step preceding leukocyte infiltration. This may provide an explanation for the escape of tumors from immune surveillance. 




\section{0}

\section{GENERAL DISCUSSION}

Leukocyte-endothelium interactions in postcapillary venules and the subsequent infiltration of leukocytes into inflamed tissue are important features in the defence reaction of our body against, for example, bacteria and viruses. Next to this defending role, leukocytes can be involved in the pathogenesis of diseases. For example, in cystic fibrosis and arthritis, and in chronic sequelae of acute infections, such as meningitis, leukocytes are in fact overzealous and their infiltration into tissues plays an important role in the course of these diseases (Lasky, 1992; Brown, 1997). Better insight into the mechanism(s) underlying leukocyte-endothelium interactions and the way in which these interactions can be modulated is of interest for understanding disease processes and for the possible development of new drugs. Induction of leukocyte-endothelium interactions is desired when the normal defence reaction is inadequate; inhibition of leukocyte-endothelium interactions may be beneficial if leukocyte infiltration has to be prevented.

In the present thesis the ability of various substances or cells to modulate leukocyte-endothelium interactions was investigated in vivo. Up till now a substantial number of the studies dealing with this aspect has been performed only in vitro. In vivo leukocyte-endothelium interactions can be observed spontaneously in, for example, skin venules (Janssen et al., 1994), but the interactions have to be induced in other tissues. In most of the studies performed in this thesis we observed leukocyte-endothelium interactions in postcapillary venules in the mesentery. In this tissue leukocyte-endothelium interactions are induced by surgical exteriorization of the tissue. The mesentery is well suited for intravital microscopic observation of blood cell-vessel wall interactions. Firstly, it is thin and translucent, allowing observation under transillumination, providing microscopic images with high contrast. Secondly, although abdominal surgery is needed, the mesentery can be exposed with minimal handling of the tissue itself. Thirdly, it has a low level of vasoactivity: in rabbits no changes in diameter or red blood cell velocity are found after topical application of vasoactive substances such as adenosine, noradrenaline, potassium chloride, a nitric oxide (NO) donor, or a NO synthesis inhibitor (Broeders et al., 1998). This allowed us to specifically study the modulating effect of substances on leukocyte-endothelium interactions, without the interference of changes in fluid dynamics.

The results described in chapter 5 and 6 of the present thesis do not support the observation of Kubes and colleagues (Kubes and Kanwar, 1994) that induction of leukocyte rolling is dependent on mast cell degranulation and the subsequent release of histamine, which induces redistribution of P-selectin to the endothelial cell surface. We show that in the rabbit mesentery leukocyte rolling can be induced, despite the fact that no mast cells are present in this tissue. Moreover, the fact that in rabbits histamine receptor antagonists do not influence leukocyte rolling indicates that histamine originating from cells other than mast cells, such as basophilic granulocytes, is not responsible for the induction of leukocyte rolling in the mesentery of this species either (chapter 5 ). In rats, the mast cell stabilizer cromoglycate did not 
reduce leukocyte rolling in any of the four strains studied, whereas the subsequent superfusion of the mesentery with histamine resulted in an increase in leukocyte rolling in Sprague-Dawley rats only (chapter 6). Our findings are in agreement with the observation of Kosaka (1997) that the induction of leukocyte-endothelium interactions by inhibitors of nitric oxide synthase, which is supposed to be a mast cell mediated event (Kubes et al., 1993; Gaboury et al., 1996), also occurs in mast cell deficient rats (Kosaka et al., 1997). Therefore, the role of mast cells in the induction of leukocyte-endothelium interactions is questionable. Mast cells, however, may be involved in the chemotaxis of leukocytes (Raud et al., 1989).

$\mathrm{P}$-selectin is the only selectin involved in leukocyte rolling in the first hour after exteriorization of the rabbit mesentery (chapter 7 ). This initial role of P-selectin in leukocyte rolling has also been observed in the mouse cremaster muscle (Ley et al., 1995). In situ, leukocytes do not interact with the vessel wall in rabbit mesentery, as histologically determined (Fiebig et al., 1991). However, within 5 minutes after gentle exteriorization of the tissue the concentration of leukocytes in postcapillary venules is increased, probably due to the interaction of leukocytes with the endothelium; leukocyte rolling accounts for the major part of this leukocyte accumulation (Fiebig et al., 1991). Therefore, handling of the rabbit mesentery rapidly induces leukocyte rolling. This also seems to hold for rat mesentery (Ley, 1994) and other tissues like the hamster cheek pouch (Atherton and Born, 1972). Considering the rate of onset of leukocyte rolling, it might predominantly be a P-selectin mediated process, since Pselectin can be redistributed to the endothelial cell surface within a few minutes (Hattori et al., 1989; Geng et al., 1990). The mechanisms responsible for this quick redistribution of $\mathrm{P}$-selectin to the endothelial cell surface remain to be established. As stated above, mast cell degranulation and histamine release are not involved in the induction of leukocyte-endothelium interactions due to tissue handling. In vitro such substances as thrombin, plasmin and oxygen radicals have been shown to induce Pselectin redistribution (see references in chapter 2 and 6). It cannot be excluded that P-selectin can be induced by mechanical stimuli such as tissue manipulation or changes in shear stress, as has been described for ICAM-1 (Malek and Izumo, 1994; Morigi et al., 1995).

In contrast to P-selectin, L-selectin is mainly involved in leukocyte rolling in the second hour after exteriorization of the rabbit mesentery (chapter 7). This finding is comparable to that in the mouse cremaster muscle in which rolling is also largely Lselectin dependent in the second and not in the first hour after surgery (Ley et al., 1995). Von Andrian and colleagues demonstrated that L-selectin plays an important role in leukocyte rolling in the rabbit mesentery not only in the second hour, but also in the first hour after exteriorization of the tissue (von Andrian et al., 1991). One should realize, however, that they based their conclusions on the observation of only 6 venules that showed an enormous variability in baseline level of leukocyte rolling (14-170 cells/min), whereas in our study 41 vessels with baseline levels between 5 and 50 rolling leukocytes/min were studied. It is conceivable that the relative contribution of different adhesion molecules changes with the absolute level of leukocyte rolling. 
An interesting finding described in this thesis is the involvement of E-selectin in leukocyte rolling in the second hour after exteriorization of the rabbit mesentery. In the mouse cremaster muscle Ley and colleagues did not find evidence for involvement of $\mathrm{E}$-selectin in the first two hours after tissue handling (Ley et al., 1995). Expression of E-selectin requires protein synthesis; in vitro, maximal cytokine induced expression of E-selectin on endothelial cells is reached between 2 and 4 hours (Bevilacqua et al., 1989). Up till now expression of E-selectin in rabbit mesenteric venules has only been demonstrated 4 hours after intraperitoneal injection of interleukin-1 (Olofsson et al., 1994). However, our findings show that gentle handling of the mesentery appears to be sufficient to activate endothelial cells to express $\mathrm{E}$-selectin within 2 hours without the need to add cytokines. This reaction may be tissue dependent. In fact, in skin E-selectin may be constitutively present on endothelial cells (Keelan et al., 1994; Janssen, 1999).

In this thesis it was investigated whether the involvement of selectins in leukocyte rolling can be modulated. The findings of chapter 7 show that about one hour after exteriorization of the mesentery a thromboembolic reaction reduces leukocyte rolling. probably due to inhibition of P-selectin and adhesion molecules other than selectins downstream of the thrombus. In the second hour adhesion molecules other than Pselectin are involved in the reduction in leukocyte rolling downstream of the thrombus. It is possible that partial inhibition of E- and L-selectin is involved in this process, but the involvement of other adhesion molecules than the ones studied is more likely. The mechanism behind the reduced bio-availability of P-selectin for leukocyte rolling downstream of a thrombus in the first hour after tissue exteriorization is as yet unclear. Substances could be released that induce rapid internalization of P-selectin (Subramaniam et al., 1993; Vestweber and Blanks, 1999) or shedding of P-selectin. Although shedding has not been described for endothelial P-selectin, soluble P-selectin in plasma has been demonstrated to be increased in several diseases (for review see (Gearing and Newman, 1993)), indicating that this adhesion molecule can be released into the circulation. Although it has been shown that platelets are the major source of soluble P-selectin, endothelial cell activation can also increase its concentration (Fijnheer et al., 1997). Another hypothetical mechanism by which the bio-availability of P-selectin for leukocyte rolling could have been diminished is coverage of endothelial cells by activated platelets immediately downstream of a thrombus, provided that the platelets do not express P-selectin, because in that case leukocytes are able to roll on these platelets (Buttrum et al., 1993; Yeo et al., 1994; Kuijper et al., 1996). However, since P-selectin does not seem to be expressed by platelets participating in the embolization phase of a thromboembolic reaction (chapter 7), adhesion of these platelets to the endothelium may have contributed to the thromboembolism induced reduction in leukocyte rolling. Another implication of the absence of P-selectin expression on 'embolizing' platelets is that it precludes the binding of such platelets to P-selectin ligands on leukocytes (Hamburger and McEver, 1990; Nash et al., 1996) as a possible mechanism for the observed reduction in leukocyte rolling. One can speculate that the clinical importance of the thromboembolism induced reduction in leukocyte rolling is 
prevention of the formation of atheromatous plaques, which contain monocytes and neutrophils (Nash et al., 1996), although this phenomenon has only been described in large vessels and not in the microcirculation.

A thromboembolism induced reduction in leukocyte rolling might also be the origin of the decrease in leukocyte rolling following infusion of sterile saline through a local cannula in a side branch of the mesenteric artery feeding the rabbit mesenteric segment under observation (chapter 4). At the tip of the cannula thrombotic processes may occur due to endothelial damage and/or platelet activation. When infusion starts substances released by cells involved in such thrombotic processes may be advanced into the circulation and influence the downstream endothelium in such a way that leukocyte rolling is reduced. Since leukocyte rolling is, among others, dependent on local pH and the extracellular calcium concentration (Geng et al., 1991; Anostario et al., 1994), one could argue that local infusion of fluids dilutes the blood and, hence, changes such parameters, thereby influencing leukocyte rolling. In our experiments, however, the dilution of the blood downstream of the local cannula was only $2-3 \%$. Therefore, it seems unlikely that changes in $\mathrm{pH}$ or calcium concentration do account for the reduction in leukocyte rolling as induced by local saline infusion.

Previous experiments have shown that the thromboembolism induced reduction in leukocyte rolling was attenuated when the rabbits were pretreated with aspirin, but not when pretreated with a thromboxane $\mathrm{A}_{2}$ receptor antagonist (oude Egbrink et al., 1992). These findings suggest that prostaglandins other than thromboxane $A_{2}$ produced by activated platelets and/or damaged vascular cells are involved in the thromboembolism induced reduction in leukocyte rolling. To evaluate the ability of prostaglandins to inhibit leukocyte rolling we studied the effects of $P G E_{1}, P G E_{2}$, and $\mathrm{PGI}_{2}$ on leukocyte rolling in the absence of a thromboembolic reaction (chapter 8 ). The concentrations used were based on literature data on prostaglandin production by activated endothelial cells in vitro. In these concentrations the three prostaglandins did not influence leukocyte rolling when tested separately or together in a cocktail. It cannot be excluded, however, that these prostaglandins play a role in the thromboembolism induced reduction in leukocyte rolling, because in the presence of activated platelets and/or damaged endothelial cells their concentrations in vivo may be higher than those tested. The prostaglandins may also act differently in cooperation with other prostaglandins or mediators released by the damaged vascular wall.

The fact that neither $\mathrm{PGE}_{1}, \mathrm{PGE}_{2}, \mathrm{PGI}_{2}$ alone, nor in combination influences leukocyte rolling (chapter 8 ) is in accordance with our own unpublished observation that aspirin, in a concentration inhibiting the production of all vascular prostaglandins, does not influence leukocyte rolling (Figure 10.1). However, our finding that the prostaglandins tested do not inhibit leukocyte rolling and adhesion, does not preclude the possibility that prostaglandins influence subsequent steps in the response of leukocytes to an activating stimulus, including diapedesis and leukocyte infiltration into the tissue, as well as oxygen radical production and enzyme release. It has been shown in several in vitro and ex vivo experiments that these 

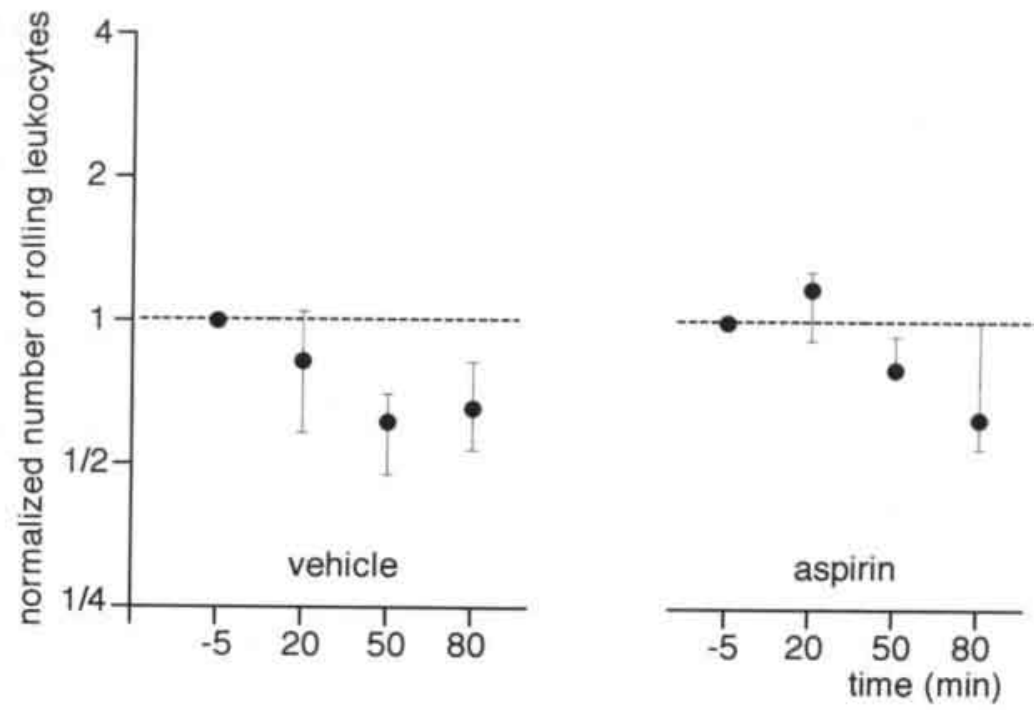

Figure 10.1. Effect of aspirin on leukocyte rolling in rabbit mesenteric venules. After baseline recordings $(t=-5)$ aspirin $(100 \mathrm{mg} / \mathrm{kg})$ or vehicle were intravenously injected $(t=0)$ and recordings were made at $t=20,50$, and 80 minutes following injection. Medians and interquartile ranges are presented. No differences were observed between the aspirin and the vehicle group.

latter leukocyte functions can be inhibited by prostaglandins (Fantone et al., 1984; Gryglewski et al., 1987). In addition, since we investigated the effect of prostaglandins on leukocyte rolling as induced by handling of the tissue only, i.e., a relatively moderate stimulus, it cannot be excluded that different results would have been obtained when leukocyte rolling had been induced by such other stimuli as ischemia/reperfusion. Furthermore, the beneficial effects of prostaglandins in such clinical situations as peripheral vascular diseases (Altstaedt et al., 1993; Wigley et al., 1994; Veale et al., 1995) or ischemia/reperfusion (Simpson et al., 1987; Simpson et al., 1988; Hohlfeld et al., 1993; Thomson et al., 1994; Natori et al., 1997), may also be partly due to vasodilating effects or to inhibition of platelet aggregation, instead of inhibiting effects on leukocytes.

Another way of modulation of leukocyte-endothelium interactions can be found in tumor microvessels: the tumor derived angiogenic factors bFGF and VEGF inhibit leukocyte adhesion (chapter 9). This is considered to be a favorable situation for tumor cells: the same substances that induce the production of new vessels and, therefore, provide the tumor cells with nutritional substances, reduce leukocyte adhesion and most probably the subsequent leukocyte infiltration. This process may explain how tumors escape from immune surveillance. Therefore, inhibition of angiogenesis may not only be capable of inhibiting the formation of new vessels, but also of preventing the escape from immune surveillance. This is indicative of a new anti-cancer modality. In fact, in vitro it has been shown that the angiogenesis inhibiting agent platelet factor- 4 is able to prevent or even to restore the bFGF 
induced reduction of leukocyte adhesion (Griffioen et al., 1999). In future research the effects of such angiogenesis inhibitors on leukocyte-endothelium interactions have to be investigated in vivo.

In conclusion, the present thesis shows that leukocyte-endothelium interactions can be modulated in vivo by several cells and substances. We demonstrated that a thromboembolic reaction reduces leukocyte rolling by inhibition of the bio-availability of P-selectin and/or adhesion molecules other than selectins, dependent on the time after tissue exteriorization, and that angiogenic factors inhibit leukocyte adhesion in vivo, probably also due to reduced expression of adhesion molecules. By contrast, mast cells and prostaglandins, which have been suggested in literature to be able to modulate leukocyte-endothelium interactions, have no effect on leukocyte rolling or adhesion in rabbit mesenteric venules in vivo. Knowledge of the modulating effects of substances on leukocyte-endothelium interactions in vivo is of importance for the development of new anti- and proinflammatory drugs. 


\section{REFERENCES}

Abbassi O., Kishimoto T.K., Mclntire L.V., Anderson D.C. and Smith C.W. E-selectin supports neutrophil rolling in vitro under conditions of flow. J Clin Invest 92:2719-2730, 1993.

Abelson M.B., Udell I.J. and Weston J.H. Conjunctival eosinophils in compound $48 / 80$ rabbit model. Arch Ophthalmol 101:631-633, 1983.

Adams D.H. and Shaw S. Leucocyte-endothelial interactions and regulation of leucocyte migration. Lancet 343:831-836, 1994.

Albelda S.M. and Buck C.A. Integrins and other cell adhesion molecules. Faseb J 4:2868-2880, 1990.

Allport J.R., Ding H.T., Ager A., Steeber D.A., Tedder T.F. and Luscinskas F.W. L-selectin shedding does not regulate human neutrophil attachment, rolling, or transmigration across human vascular endothelium in vitro. J Immunol 158:4365-4372, 1997.

Altstaedt H.O., Berzewski B., Breddin H.K., Brockhaus W., Bruhn H.D., Cachovan M., Diehm C., Dorrler J., Franke C.S., Gruss J.D. et al. Treatment of patients with peripheral arterial occlusive disease Fontaine stage IV with intravenous iloprost and PGE1: a randomized open controlled study. Prostaglandins Leukot Essent Fatty Acids 49:573-578, 1993.

Amoroso A., Del Porto F., Di Monaco C., Manfredini P. and Afeltra A. Vascular endothelial growth factor: a key mediator of neoangiogenesis. A review. Eur Rev Med Pharmacol Sci 1:17-25, 1997.

Anderson D.C. and Springer T.A. Leukocyte adhesion deficiency: an inherited defect in the Mac-1, LFA-1, and p150,95 glycoproteins. Annu Rev Med 38:175-194, 1987.

Anostario M., Jr. and Huang K.S. Modulation of E-selectin structure/function by metal ions. Studies on limited proteolysis and metal ion regeneration. J Biol Chem 270:8138-8144, 1995.

Anostario M., Jr., Li S.H. and Huang K.S. A ligand binding assay for E-selectin. Anal Biochem 221:317-322, 1994.

Arca M.J., Mule J.J. and Chang A.E. Genetic approaches to adoptive cellular therapy of malignancy. Semin Oncol 23:108-117, 1996.

Arfors K.E. and Bergqvist D. Platelet aggregability in microvascular haemostasis and the effect of local inflammation. Haemostasis 7:46-53, 1978.

Armstead V.E., Minchenko A.G., Schuhl R.A., Hayward R., Nossuli T.O. and Lefer A.M. Regulation of P-selectin expression in human endothelial cells by nitric oxide. Am J Physiol 273: $\mathrm{H} 740-\mathrm{H} 746,1997$.

Amaout M.A. Structure and function of the leukocyte adhesion molecules CD11/CD18. Blood 75:1037-1050, 1990.

Asako H., Kurose I., Wolf R., DeFrees S., Zheng Z.L., Phillips M.L., Paulson J.C. and Granger D.N. Role of $\mathrm{H} 1$ receptors and P-selectin in histamine-induced leukocyte rolling and adhesion in postcapillary venules. J Clin Invest 93:1508-1515, 1994.

Atherton A. and Born G.V. Quantitative investigations of the adhesiveness of circulating polymorphonuclear leucocytes to blood vessel walls. J Physiol Lond 222:447-474, 1972.

Baez B. An open cremaster muscle preparation for the study of blood vessels by in vivo microscopy. Microvasc Res 5:384-394, 1973.

Bartlett M.R. and Parish C.R. Venular and arterial endothelial cells differ in their expression of adhesion molecules and in their ability to degrade the subendothelial basement membrane. Endothelium 2:331-338, 1995.

Bates E.J. Eicosanoids, fatty acids and neutrophils: their relevance to the pathophysiology of disease. Prostaglandins Leukot Essent Fatty Acids 53:75-86, 1995.

Belch J.J., Capell H.A., Cooke E.D., Kirby J.D., Lau C.S., Madhok R., Murphy E. and Steinberg M. Oral iloprost as a treatment for Raynaud's syndrome: a double blind multicentre placebo controlled study. Ann Rheum Dis 54:197-200, 1995.

Bengisun J., Koksoy C., Bengisun J.S., Bayraktaroglu G., Camur A. and Aras N. Ischemia and reperfusion injury: prevention of pulmonary hypertension and leukosequestration following 
lower limb ischemia. Prostaglandins Leukot Essent Fatty Acids 56:117-120, 1997.

Berends C., Dijkhuizen B., de Monchy J.G., Dubois A.E., Gerritsen J. and Kauffman H.F. Inhibition of PAF-induced expression of CD11b and shedding of L-selectin on human neutrophils and eosinophils by the type IV selective PDE inhibitor, rolipram. Eur Respir J 10:1000-1007, 1997.

Berg E.L., McEvoy L.M., Berlin C., Bargatze R.F. and Butcher E.C. L-selectin-mediated lymphocyte rolling on MAdCAM-1. Nature 366:695-698, 1993.

Bergqvist D. and Arfors K.E. Microvascular haemostasis and the effect of local stimulation and inhibition of platelet function. An experimental study in rabbits. Thromb Haemost 36:133139, 1976.

Bernstein J.A. and Lawrence I.D. The mast cell: a comprehensive, updated review. Allergy Proc 11:209-223, 1990.

Beuk R.J., Heineman E., Tangelder G.J., Kurvers H.A.J.M., Bonke H.J. and oude Egbrink M.G.A. Effects of different durations of total warm ischemia of the gut on rat mesenteric microcirculation. J Surg Res 73:14-23, 1997.

Beuk R.J., oude Egbrink M.G.A., Kurvers H.A.J.M., Bonke H.J., Tangelder G.J. and Heineman E. Ischemia/reperfusion injury in rat mesenteric venules: red blood cell velocity and leukocyte rolling. J Pediatr Surg 31:512-515, 1996.

Bevilacqua M.P., Pober J.S., Mendrick D.L. and Cotran R.S. Identification of an inducible endothelial-leukocyte adhesion molecule. Proc Natl Acad Sci U S A 84:9238-9242, 1987.

Bevilacqua M.P., Stengelin S., Gimbrone M.A., Jr. and Seed B. Endothelial leukocyte adhesion molecule 1: an inducible receptor for neutrophils related to complement regulatory proteins and lectins. Science 243:1160-1165, 1989.

Bicknell R. Heterogeneity of the endothelial cell. Behring Inst Mitt 92:1-7, 1993.

Bienvenu K., Harris N. and Granger D.N. Modulation of leukocyte migration in mesenteric interstitium. Am J Physiol 267:H1573-H1577, 1994.

Borgström P., Hughes G.K., Hansell P., Wolitsky B.A. and Sriramarao P. Leukocyte adhesion in angiogenic blood vessels. Role of E-selectin, P-selectin, and beta2 integrin in lymphotoxin-mediated leukocyte recruitment in tumor microvessels. J Clin Invest 99:22462253, 1997.

Boukerche H., Ruchaud Sparagano M.H., Rouen C., Brochier J., Kaplan C. and McGregor J.L. A monoclonal antibody directed against a granule membrane glycoprotein (GMP140/PADGEM, P-selectin, CD62P) inhibits ristocetin-induced platelet aggregation. $\mathrm{Br} J$ Haematol 92:442-451, 1996.

Broeders M.A., Tangelder G.J., Slaaf D.W., Reneman R.S. and oude Egbrink M.G. Endogenous nitric oxide protects against thromboembolism in venules but not in arterioles. Arterioscler Thromb Vasc Biol 18:139-145, 1998.

Brown E. Neutrophil adhesion and the therapy of inflammation. Semin Hematol 34:319-326, 1997.

Brown N.J. and Reed M.W. Leucocyte interactions with the mouse cremaster muscle microcirculation in vivo in response to tumour-conditioned medium. Br J Cancer 75:993-999, 1997.

Bruehl R.E., Springer T.A. and Bainton D.F. Quantitation of L-selectin distribution on human leukocyte microvilli by immunogold labeling and electron microscopy. J Histochem Cytochem 44:835-844, 1996.

Butcher E.C. Leukocyte-endothelial cell recognition: three (or more) steps to specificity and diversity. Cell 67:1033-1036, 1991.

Buttrum S.M., Hatton R. and Nash G.B. Selectin-mediated rolling of neutrophils on immobilized platelets. Blood 82:1165-1174, 1993.

Celi A., Lorenzet R., Furie B. and Furie B.C. Platelet-leukocyte-endothelial cell interaction on the blood vessel wall. Semin Hematol 34:327-335, 1997.

Chang A.E. and Shu S. Current status of adoptive immunotherapy of cancer. Crit Rev Oncol Hematol 22:213-228, 1996. 
Chole R.A. and Kodama K. Comparative histology of the tympanic membrane and its relationship to cholesteatoma. Ann Otol Rhinol Laryngol 98:761-766, 1989.

Cines D.B., Pollak E.S., Buck C.A., Loscalzo J., Zimmerman G.A., McEver R.P., Pober J.S., Wick T.M., Konkle B.A., Schwartz B.S., Barnathan E.S., McCrae K.R., Hug B.A., Schmidt A.M. and Stern D.M. Endothelial cells in physiology and in the pathophysiology of vascular disorders. Blood 91:3527-3561, 1998.

Clark J.M., Altman G. and Fromowitz F.B. Basophil hypersensitivity response in rabbits. Infect Immun 15:305-312, 1977.

Coker S.J., Clarke B. and Zeitlin I.J. Radioimmunoassay techniques for the determination of the local release of prostaglandins and thromboxanes. J Pharmacol Methods 7:207-217. 1982.

Collins T. Adhesion molecules in leukocyte emigration. Scientific American nov/dec:28-37. 1995.

Crockett-Torabi E., Sulenbarger B., Smith C.W. and Fantone J.C. Activation of human neutrophils through L-selectin and Mac-1 molecules. J Immunol 154:2291-2302, 1995.

Crockett-Torabi E. Selectins and mechanisms of signal transduction. J Leukoc Biol 63:1-14, 1998.

Datta Y.H., Romano M., Jacobson B.C., Golan D.E., Serhan C.N. and Ewenstein B.M. Peptidoleukotrienes are potent agonists of von Willebrand factor secretion and P-selectin surface expression in human umbilical vein endothelial cells. Circulation 92:3304-3311, 1995.

Davenpeck K.L., Gauthier T.W. and Lefer A.M. Inhibition of endothelial-derived nitric oxide promotes P-selectin expression and actions in the rat microcirculation. Gastroenterology 107:1050-1058, 1994.

Dellian M., Abels C., Kuhnle G.E. and Goetz A.E. Effects of photodynamic therapy on leucocyte-endothelium interaction: differences between normal and tumour tissue. $\mathrm{Br} J$ Cancer 72:1125-1130, 1995.

Dellian M., Witwer B.P., Salehi H.A., Yuan F. and Jain R.K. Quantitation and physiological characterization of angiogenic vessels in mice: effect of basic fibroblast growth factor. vascular endothelial growth factor/vascular permeability factor, and host microenvironment. Am J Pathol 149:59-71, 1996.

den Ottolander G.J. Diagnostisch Kompas 1997. Utrecht: Boekhoven-Bosch, 1997

Derhaag J.G., Duijvestijn A.M., Emeis J.J., Engels W. and van Breda Vriesman P.J. Production and characterization of spontaneous rat heart endothelial cell lines. Lab Invest 74:437-451, 1996.

Derhaag J.G., Duijvestijn A.M. and Van Breda Vriesman P.J. Heart EC respond heterogeneous on cytokine stimulation in ICAM-1 and VCAM-1, but not in MHC expression. A study with 3 rat heart endothelial cell (RHEC) lines. Endothelium 5:307-319, 1997.

Desai K.H., Sato R., Schauble E., Barsh G.S., Kobilka B.K. and Bernstein D. Cardiovascular indexes in the mouse at rest and with exercise: new tools to study models of cardiac disease. Am J Physiol 272:H1053-H1061, 1997.

Detmar M., Brown L.F., Schon M.P., Elicker B.M., Velasco P., Richard L., Fukumura D., Monsky W., Claffey K.P. and Jain R.K. Increased microvascular density and enhanced leukocyte rolling and adhesion in the skin of VEGF transgenic mice. J Invest Dermatol 111:1-6, 1998.

Doré M., Korthuis R.J., Granger D.N., Entman M.L. and Smith C.W. P-selectin mediates spontaneous leukocyte rolling in vivo. Blood 82:1308-1316, 1993.

Dore-Duffy P., Berube M.L., Siok C. and Zurier R.B. Oral administration of prostaglandin E2 to humans: effects on peripheral blood leukocyte function. J Lab Clin Med 104:283-291, 1984.

Dvorak H.F. and Dvorak A.M. Immunohistological characterization of inflammatory cells that infiltrate tumors. In: Haskill S, ed. Tumor immunity in prognosis. New York: Marcel Dekker, Inc., 1982: 279-307.

El Maradny E., Kanayama N., Halim A., Maehara K., Sumimoto K. and Terao T. Biochemical changes in the cervical tissue of rabbit induced by interleukin-8, interleukin-1beta, dehydroepiandrosterone sulphate and prostaglandin E2: a comparative study. Hum Reprod 
11:1099-1104, 1996.

Engelberts I., van Hoof S.C., Samyo S.K., Buurman W.A. and van der Linden C.J. Generalized inflammation during peritonitis evidenced by intracutaneous E-selectin expression. Clin Immunol Immunopathol 65:330-334, 1992.

Ennis M., Lorenz W., Gerland W. and Heise J. Isolation of mast cells from rabbit lung and liver: comparison of histamine release induced by the hypnotics Althesin and propanidid. Agents Actions 20:219-222, 1987.

Etzioni A., Frydman M., Pollack S., Avidor I. and Phillips M.L. Brief report: recurrent severe infections caused by a novel leukocyte adhesion deficiency. N Engl J Med 327:1789-1792, 1992.

Faint R.W. Platelet-neutrophil interactions: their significance. Blood Rev 6:83-91, 1992.

Fantone J.C., Marasco W.A., Elgas L.J. and Ward P.A. Stimulus specificity of prostaglandin inhibition of rabbit polymorphonuclear leukocyte lysosomal enzyme release and superoxide anion production. Am J Pathol 115:9-16, 1984.

Fijnheer R., Frijns C.J., Korteweg J., Rommes H., Peters J.H., Sixma J.J. and Nieuwenhuis H.K. The origin of P-selectin as a circulating plasma protein. Thromb Haemost 77:10811085, 1997.

Fisher C.A., Kappa J.R., Sinha A.K., Cottrell E.D., Reiser H.J. and Addonizio V.P. Comparison of equimolar concentrations of iloprost, prostacyclin, and prostaglandin E1 on human platelet function. J Lab Clin Med 109:184-190, 1987.

FitzGerald G.A., Catella F. and Oates J.A. Eicosanoid biosynthesis in human cardiovascular disease. Hum Pathol 18:248-252, 1987.

Fukumura D., Salehi H.A., Witwer B., Tuma R.F., Melder R.J. and Jain R.K. Tumor necrosis factor alpha-induced leukocyte adhesion in normal and tumor vessels: effect of tumor type, transplantation site, and host strain. Cancer Res 55:4824-4829, 1995.

Gaboury J.P., Johnston B., Niu X.F. and Kubes P. Mechanisms underlying acute mast cellinduced leukocyte rolling and adhesion in vivo. J Immunol 154:804-813, 1995.

Gaboury J.P., Niu X.F. and Kubes P. Nitric oxide inhibits numerous features of mast cellinduced inflammation. Circulation 93:318-326, 1996.

Gahmberg C.G., Tolvanen M. and Kotovuori P. Leukocyte adhesion--structure and function of human leukocyte beta2-integrins and their cellular ligands. Eur J Biochem 245:215-232, 1997.

Gahmberg C.G., Valmu L., Fagerholm S., Kotovuori P., Ihanus E., Tian L. and Pessa Morikawa T. Leukocyte integrins and inflammation. Cell Mol Life Sci 54:549-555, 1998.

Gallatin W.M., Weissman I.L. and Butcher E.C. A cell-surface molecule involved in organspecific homing of lymphocytes. Nature 304:30-34, 1983.

Galli S.J. New concepts about the mast cell. N Engl J Med 328:257-265, 1993.

Gearing A.J. and Newman W. Circulating adhesion molecules in disease. Immunol Today 14:506-512, 1993.

Gebuhrer V., Murphy J.F., Bordet J.C., Reck M.P. and McGregor J.L. Oxidized low-density lipoprotein induces the expression of P-selectin (GMP140/PADGEM/CD62) on human endothelial cells. Biochem J 306:293-298, 1995.

Geng J.G., Bevilacqua M.P., Moore K.L., Mclntyre T.M., Prescott S.M., Kim J.M., Bliss G.A., Zimmerman G.A. and McEver R.P. Rapid neutrophil adhesion to activated endothelium mediated by GMP-140. Nature 343:757-760, 1990.

Geng J.G., Moore K.L., Johnson A.E. and McEver R.P. Neutrophil recognition requires a $\mathrm{Ca}(2+)$-induced conformational change in the lectin domain of GMP-140. J Biol Chem 266:22313-22318, 1991.

Gerritsen M.E. and Cheli C.D. Arachidonic acid and prostaglandin endoperoxide metabolism in isolated rabbit and coronary microvessels and isolated and cultivated coronary microvessel endothelial cells. J Clin Invest 72:1658-1671, 1983.

Gerritsen M.E. Functional heterogeneity of vascular endothelial cells. Biochem Pharmacol 36:2701-2711. 1987. 
Gerritsen M.E. Physiological and pathophysiological roles of eicosanoids in the microcirculation. Cardiovasc Res 32:720-732, 1996.

Goodwin J.S. Are prostaglandins proinflammatory, antiinflammatory, both or neither? J Rheumatol Suppl 28:26-29, 1991.

Grant S.M. and Goa K.L. Iloprost. A review of its pharmacodynamic and pharmacokinetic properties, and therapeutic potential in peripheral vascular disease, myocardial ischaemia and extracorporeal circulation procedures. Drugs 43:889-924, 1992.

Griffioen A.W., Damen C.A., Blijham G.H. and Groenewegen G. Tumor angiogenesis is accompanied by a decreased inflammatory response of tumor-associated endothelium. Blood 88:667-673, 1996a.

Griffioen A.W., Damen C.A., Martinotti S., Blijham G.H. and Groenewegen G. Endothelial intercellular adhesion molecule-1 expression is suppressed in human malignancies: the role of angiogenic factors. Cancer Res 56:1111-1117, $1996 \mathrm{~b}$.

Griffioen A.W. Phenotype of the tumor vasculature; cell adhesion as a target for tumor therapy. the Cancer Journal 10:249-255, 1997.

Griffioen A.W., Tromp S.C. and Hillen H.F. Angiogenesis modulates the tumour immune response. Int J Exp Pathol 79:363-368, 1998.

Griffioen A.W., Damen C.A., Mayo K.H., Barendsz-Janson A.F., Martinotti S., Blijham G.H. and Groenewegen $\mathrm{G}$. Angiogenesis inhibitors overcome tumor induced endothelial cell anergy. Int J Cancer 80:315-319, 1999.

Gryglewski R.J., Szczeklik A. and Wandzilak M. The effect of six prostaglandins, prostacyclin and iloprost on generation of superoxide anions by human polymorphonuclear leukocytes stimulated by zymosan or formyl-methionyl-leucyl-phenylalanine. Biochem Pharmacol 36:4209-4213, 1987.

Guyton A.C. and Hall J.E. Textbook of medical physiology. (9 ed.) Philadelphia, U.S.A., W.B. Saunders Company, 1996

Hamburger S.A. and McEver R.P. GMP-140 mediates adhesion of stimulated platelets to neutrophils. Blood 75:550-554, 1990.

Hattori R., Hamilton K.K., Fugate R.D., McEver R.P. and Sims P.J. Stimulated secretion of endothelial von Willebrand factor is accompanied by rapid redistribution to the cell surface of the intracellular granule membrane protein GMP-140. J Biol Chem 264:7768-7771, 1989.

Haynes B.F. and Fauci A.S. The immune system. In: Wilson JD, Braunwald E, Isselbacher KJ, ed. Harrison's principles of internal medicine. New York, McGraw-Hill, Inc., 1991: 76-86.

Hellwig S.M., Damen C.A., van Adrichem N.P., Blijham G.H., Groenewegen G. and Griffioen A.W. Endothelial CD34 is suppressed in human malignancies: role of angiogenic factors. Cancer Lett 120:203-211, 1997.

Higgs G.A., Moncada S. and Vane J.R. Prostacyclin reduces the number of 'slow moving' leucocytes in hamster cheek pouch venules. J Physiol Lond 280:55P-56P, 1978.

Hohlfeld T., Strobach H. and Schrör K. Stimulation of endogenous prostacyclin protects the reperfused pig myocardium from ischemic injury. J Pharmacol Exp Ther 264:397-405, 1993.

House S.D. and Lipowsky H.H. Leukocyte-endothelium adhesion: microhemodynamics in mesentery of the cat. Microvasc Res 34:363-379, 1987.

Huang Z., Li S. and Korngold R. Immunoglobulin superfamily proteins: structure, mechanisms, and drug discovery. Biopolymers 43:367-382, 1997.

Imhof B.A. and Dunon D. Basic mechanism of leukocyte migration. Horm Metab Res 29:614621, 1997.

Intaglietta M. and Tompkins W.R. Microvascular measurements by video image shearing and splitting. Microvasc Res 5:309-312, 1973.

Isenberg W.M., McEver R.P., Shuman M.A. and Bainton D.F. Topographic distribution of a granule membrane protein (GMP-140) that is expressed on the platelet surface after activation: an immunogold-surface replica study. Blood Cells 12:191-204, 1986.

Jain R.K., Koenig G.C., Dellian M., Fukumura D., Munn L.L. and Melder R.J. Leukocyteendothelial adhesion and angiogenesis in tumors. Cancer Metastasis Rev 15:195-204, 1996. 
Janssen G.H.G.W., Tangelder G.J., oude Egbrink M.G.A. and Reneman R.S. Spontaneous leukocyte rolling in venules in untraumatized skin of conscious and anesthetized animals. Am J Physiol 267:H1199-H1204, 1994.

Janssen G.H.G.W., Tangelder G.J., oude Egbrink M.G.A. and Reneman R.S. Different effects of anesthetics on spontaneous leukocyte rolling in rat skin. Int J Microcirc Clin Exp 17:305313, 1997.

Janssen G.H.G.W. Leukocyte rolling in skin venules. Thesis. Technische Universiteit Eindhoven, 1999.

Jarcho S. Cohnheim on inflammation 1. Am J Cardiol 29:247-249, 1972.

Jones D.A., Abbassi O., Mclntire L.V., McEver R.P. and Smith C.W. P-selectin mediates neutrophil rolling on histamine-stimulated endothelial cells. Biophys J 65:1560-1569, 1993.

Jones D.A., Mclntire L.V., Smith C.W. and Picker L.J. A two-step adhesion cascade for T cell/endothelial cell interactions under flow conditions. J Clin Invest 94:2443-2450, 1994.

Jones G. and Hurley J.V. The effect of prostacyclin on the adhesion of leucocytes to injured vascular endothelium. J Pathol 142:51-59, 1984.

Jonsson M., Tzanela M., Kolbeck R.C. and McCormick J.R. Hemodynamic and metabolic effects of intravenous formyl-methionyl-leucyl-phenylalanine (FMLP) in rabbits. In Vivo 11:133-139, 1997.

Jung U. and Ley K. Regulation of E-selectin, P-selectin, and intercellular adhesion molecule 1 expression in mouse cremaster muscle vasculature. Microcirculation 4:311-319, 1997.

Kaley G., Hintze T.H., Panzenbeck M. and Messina E.J. Role of prostaglandins in microcirculatory function. Adv Prostaglandin Thromboxane Leukot Res 13:27-35, 1985.

Kanamori Y., Niwa M., Kohno K., Al Essa L.Y., Matsuno H., Kozawa O. and Uematsu T. Migration of neutrophils from blood to tissue: alteration of modulatory effects of prostanoid on superoxide generation in rabbits and humans. Life Sci 60:1407-1417, 1997.

Kanayama T., Kimura Y. and Mizogami S. A new prostacyclin analog, KP-10614, inhibits platelet-polymorphonuclear leukocyte interaction and limits experimental infarct size in rat heart. J Pharmacol Exp Ther 266:344-349, 1993.

Kansas G.S. Structure and function of L-selectin. Apmis 100:287-293, 1992.

Kanwar $S$. and Kubes $P$. Mast cells contribute to ischemia-reperfusion-induced granulocyte infiltration and intestinal dysfunction. Am J Physiol 267:G316-G321, 1994.

Kawashima H., Watanabe N., Hirose M., LiY.F., Hirose J. and Miyasaka M. Vascular and nonvascular ligands for L-selectin. Cell Adhes Commun 6:135-139, 1998.

Keelan E.T., Licence S.T., Peters A.M., Binns R.M. and Haskard D.O. Characterization of Eselectin expression in vivo with use of a radiolabeled monoclonal antibody. Am J Physiol 266: $\mathrm{H} 278-\mathrm{H} 290,1994$.

Khew-Goodall Y., Butcher C.M., Litwin M.S., Newlands S., Korpelainen E.I., Noack L.M., Berndt M.C., Lopez A.F., Gamble J.R. and Vadas M.A. Chronic expression of P-selectin on endothelial cells stimulated by the T-cell cytokine, interleukin-3. Blood 87:1432-1438, 1996.

Kirkpatrick C.J., Wagner M., Hermanns I., Klein C.L., Kohler H., Otto M., van Kooten T.G. and Bittinger F. Physiology and cell biology of the endothelium: a dynamic interface for cell communication. Int J Microcirc Clin Exp 17:231-240, 1997.

Kishimoto T.K., Jutila M.A., Berg E.L. and Butcher E.C. Neutrophil Mac-1 and MEL-14 adhesion proteins inversely regulated by chemotactic factors. Science 245:1238-1241, 1989.

Kishimoto T.K., Jutila M.A. and Butcher E.C. Identification of a human peripheral lymph node homing receptor: a rapidly down-regulated adhesion molecule. Proc Natl Acad Sci U S A 87:2244-2248, 1990.

Kitayama J., Nagawa H., Yasuhara H., Tsuno N., Kimura W., Shibata Y. and Muto T. Suppressive effect of basic fibroblast growth factor on transendothelial emigration of $\mathrm{CD} 4(+)$ T-lymphocyte. Cancer Res 54:4729-4733, 1994.

Kitsis E.A., Weissmann G. and Abramson S.B. The prostaglandin paradox: additive inhibition of neutrophil function by aspirin-like drugs and the prostaglandin E1 analog misoprostol. J Rheumatol 18:1461-1465, 1991. 
Kosaka H., Seyama A. Terada N. Yeneyama H. Hirota S, and Kitamura Y, Absence of mast cell involvement in leukocyte adhesion and emigration induced by inhibition of nitric oxide synthase. Lab invest $77.575 .580,1997$.

Kotovuon P., Tontb E., Pigott R., Shepherd M., Kiso M., Hasewaga A., Renkonen R., Nortamo D. Altieri D.C. and Gahmberg C. G. The vascular E-selectin binds to the leukocyte integring CD11/CD18. Gycobiology 3:131-136, 1993.

Kozma C., Mackin W., Cummins L.M. and Mauer R. The anatomy, physiology, and the biochemistry of the rabbit. In: Weisbroth SE, Flatt RE, Kraus AL, ed. The biology of the laboratory rabbit. New York: Academic Press, Inc., 1974: 50-72.

Kubes P. Polymorphonuclear leukocyte-endothelium interactions: a role for pro-inflammatory and ant-inflammatory molecules. Can J Physiol Phamacol 71:88-97, 1993.

Kubes P., Kanwar S., Niu X.F. and Gaboury J.P. Nitric oxide synthesis inhibition induces leukocyte adhesion via superoxide and mast cells. Faseb J 7:1293-1299, 1993.

Kubes $\mathrm{P}$. and Kanwar S. Histamine induces leukocyte rolling in post-capillary venules. A P. selectin-mediated event. J Immunol 152:3570-3577, 1994.

Kuijper P.H.M., Gallardo Torres H.I., van der Linden J.A.M., Lammers J.W.J., Sixma J.J., Koenderman L. and Zwaginga J.J. Platelet-dependent primary hemostasis promotes selectin- and integrin-mediated neutrophil adhesion to damaged endothelium under flow conditions. Blood 87:3271-3281, 1996.

Kuijpers T.W., Hakkert B.C., Hart M.H. and Roos D. Neutrophil migration across monolayers of cytokine-prestimulated endothelial cells: a role for platelet-activating factor and IL-8. J Cell Biol 117:565-572, 1992a.

Kuijpers T.W., Hoogerwerf M., van der Laan L.J., Nagel G., van der Schoot C.E., Grunert F and Roos D. CD66 nonspecific cross-reacting antigens are involved in neutrophil adherence to cytokine-activated endothelial cells. J Cell Biol 118:457-466, $1992 \mathrm{~b}$.

Kuijpers T.W., Van Lier R.A., Hamann D., de Boer M., Thung L.Y., Weening R.S., Verhoeven A.J. and Roos D. Leukocyte adhesion deficiency type 1 (LAD-1)/variant. A novel immunodeficiency syndrome characterized by dysfunctional beta2 integrins. J Clin Invest 100:1725-1733, 1997.

Kunkel E.J., Jung U., Bullard D.C., Norman K.E., Wolitzky B.A., Vestweber D., Beaudet A.L. and Ley K. Absence of trauma-induced leukocyte rolling in mice deficient in both P-selectin and intercellular adhesion molecule 1. J Exp Med 183:57-65, 1996.

Kunkel E.J. and Ley K. Distinct phenotype of E-selectin-deficient mice. E-selectin is required for slow leukocyte rolling in vivo. Circ Res 79:1196-1204, 1996.

Kuzu I., Bicknell R., Fletcher C.D. and Gatter K.C. Expression of adhesion molecules on the endothelium of normal tissue vessels and vascular tumors. Lab Invest 69:322-328, 1993.

Larsen G.R., Sako D., Ahem T.J., Shaffer M., Erban J., Sajer S.A., Gibson R.M., Wagner D.D. Furie B.C. and Furie B. P-selectin and E-selectin. Distinct but overlapping leukocyte ligand specificities. J Biol Chem 267:11104-11110, 1992.

Lasky L.A. Selectins: interpreters of cell-specific carbohydrate information during inflammation. Science 258:964-969, 1992.

Lawrence M.B., Mclntire L.V. and Eskin S.G. Effect of flow on polymorphonuclear leukocyte/endothelial cell adhesion. Blood 70:1284-1290, 1987.

Lawrence M.B. and Springer T.A. Neutrophils roll on E-selectin. J Immunol 151:6338-6346, 1993.

Leeuwenberg J.F., Smeets E.F., Neefjes J.J., Shaffer M.A., Cinek T., Jeunhomme T.M., Ahern T.J. and Buurman W.A. E-selectin and intercellular adhesion molecule-1 are released by activated human endothelial cells in vitro. Immunology 77:543-549, 1992.

Levy J.H. The human inflammatory response. J Cardiovasc Pharmacol 27 Suppl 1:S31-S37. 1996.

Lewinsohn D.M., Bargatze R.F. and Butcher E.C. Leukocyte-endothelial cell recognition: evidence of a common molecular mechanism shared by neutrophils, lymphocytes, and other leukocytes. J Immunol 138:4313-4321, 1987. 
Ley K. and Gaehtgens P. Endothelial, not hemodynamic, differences are responsible for preferential leukocyte rolling in rat mesenteric venules. Circ Res 69:1034-1041, 1991.

Ley K., Cerrito M. and Arfors K.E. Sulfated polysaccharides inhibit leukocyte rolling in rabbit mesentery venules. Am J Physiol 260: H1667-H1673, 1991a.

Ley K., Gaehtgens P., Fennie C., Singer M.S., Lasky L.A. and Rosen S.D. Lectin-like cell adhesion molecule 1 mediates leukocyte rolling in mesenteric venules in vivo. Blood 77:2553-2555, $1991 \mathrm{~b}$.

Ley K., Tedder T.F. and Kansas G.S. L-selectin can mediate leukocyte rolling in untreated mesenteric venules in vivo independent of $E$ - or P-selectin. Blood 82:1632-1638, 1993.

Ley $\mathrm{K}$. Histamine can induce leukocyte rolling in rat mesenteric venules. Am J Physiol 267: $\mathrm{H} 1017-\mathrm{H} 1023,1994$.

Ley K., Bullard D.C., Arbones M.L., Bosse R., Vestweber D., Tedder T.F. and Beaudet A.L. Sequential contribution of L- and P-selectin to leukocyte rolling in vivo. J Exp Med 181:669. $675,1995$.

Ley K., Allietta M., Bullard D.C. and Morgan S. Importance of E-selectin for firm leukocyte adhesion in vivo, Circ Res 83:287-294, 1998.

Lindbom L., Xie X., Raud J. and Hedqvist P. Chemoattractant-induced firm adhesion of leukocytes to vascular endothelium in vivo is critically dependent on initial leukocyte rolling. Acta Physiol Scand 146:415-421, 1992.

Linssen M.C., Engels W., Lemmens P.J., Heijnen V.V., Van Bilsen M., Reneman R.S. and van der Vusse G.J. Production of arachidonic acid metabolites in adult rat cardiac myocytes, endothelial cells, and fibroblast-like cells. Am J Physiol 264:H973-H982, 1993.

Lorant D.E., Topham M.K., Whatley R.E., McEver R.P., Mclntyre T.M., Prescott S.M. and Zimmerman G.A. Inflammatory roles of P-selectin. J Clin Invest 92:559-570, 1993.

MacGregor R.R., Safford M. and Shalit M. Effect of ethanol on functions required for the delivery of neutrophils to sites of inflammation. J Infect Dis 157:682-689, 1988.

Malek A.M. and Izumo S. Molecular aspects of signal transduction of shear stress in the endothelial cell. J Hypertens 12:989-999, 1994.

Mason I.J. The ins and outs of fibroblast growth factors. Cell 78:547-552, 1994

Masotti G., Poggesi L., Galanti G., Trotta F. and Serneri G.G.N. Prostacyclin production in man In: Lewis PJ, O'Grady J, ed. Clinical pharmacology of prostacyclin. New York, Raven Press. 1981: 9-20.

Mayadas T.N., Johnson R.C., Rayburn H., Hynes R.O. and Wagner D.D. Leukocyte rolling and extravasation are severely compromised in P selectin-deficient mice. Cell 74:541-554, 1993.

McEver R.P. Leukocyte interactions mediated by selectins. Thromb Haemost 66:80-87, 1991.

Melder R.J., Koenig G.C., Witwer B.P., Safabakhsh N., Munn L.L. and Jain R.K. During angiogenesis, vascular endothelial growth factor and basic fibroblast growth factor regulate natural killer cell adhesion to tumor endothelium. Nat Med 2:992-997, 1996.

Mendonca V.O., Vugman I. and Jamur M.C. Maturation of aduit rat peritoneal and mesenteric mast cells. A morphological and histofluorescence study. Cell Tissue Res 243:635-639, 1986.

Meng H., Tonnesen M.G., Marchese M.J., Clark R.A., Bahou W.F. and Gruber B.L. Mast cells are potent regulators of endothelial cell adhesion molecule ICAM-1 and VCAM-1 expression. J Cell Physiol 165:40-53, 1995.

Mengelers H.J., Maikoe T., Hooibrink B., Kuypers T.W., Kreukniet J., Lammers J.W. and Koenderman L. Down modulation of L-Selectin expression on eosinophils recovered from bronchoalveolar lavage fluid after allergen provocation. Clin Exp Allergy 23:196-204, 1993.

Menger M.D. and Lehr H.A. Scope and perspectives of intravital microscopy-bridge over from in vitro to in vivo. Immunol Today 14:519-522, 1993.

Mohan C.R., Ascer E., Marini C.P., Maughan R.E. and Gennaro M. Does iloprost mediate thromboxane activity and polymorphonuclear leukocyte sequestration in ischemic skeletal muscle? J Cardiovasc Surg Torino 33:613-618, 1992.

Montrucchio G., Lupia E., De Martino A., Silvestro L., Savu S.R., Cacace G., De Filippi P.G. 
Emanuelli G. and Camussi G. Plasmin promotes an endothelium-dependent adhesion of neutrophils. Involvement of platelet activating factor and P-selectin. Circulation 93:21522160, 1996.

Moore K.L., Patel K.D., Bruehl R.E., Li F., Johnson D.A., Lichenstein H.S., Cummings R.D. Bainton D.F. and McEver R.P. P-selectin glycoprotein ligand-1 mediates rolling of human neutrophils on P-selectin. J Cell Biol 128:661-671, 1995.

Moore K.L. Structure and function of P-selectin glycoprotein ligand-1. Leuk Lymphoma 29:1-15. 1998.

Morigi M., Zoja C., Figliuzzi M., Foppolo M., Micheletti G., Bontempelli M., Saronni M., Remuzzi G. and Remuzzi A. Fluid shear stress modulates surface expression of adhesion molecules by endothelial cells. Blood 85:1696-1703, 1995.

Müller B., Schmidtke M. and Witt W. Adherence of leucocytes to electrically damaged venules in vivo. Effects of iloprost, PGE1, indomethacin, forskolin, BW $755 \mathrm{C}$, sulotroban, hirudin, and thrombocytopenia. Eicosanoids 1:13-17, 1988.

Nash G.B., Morland C., Sheikh S., Buttrum S.M. and Lalor P. Adhesion between leucocytes and platelets: rheology, mechanisms and consequences. In: Messmer K, ed. Compromised perfusion. Basel, Karger, 1996: 98-113.

Natori S., Fujii Y., Kurosawa H., Nakano A. and Shimada H. Prostaglandin E1 protects against ischemia-reperfusion injury of the liver by inhibition of neutrophil adherence to endothelial cells. Transplantation 64:1514-1520, 1997.

Neeley S.P., Hamann K.J., White S.R., Baranowski S.L., Burch R.A. and Leff A.R. Selective regulation of expression of surface adhesion molecules Mac-1, L-selectin, and VLA-4 on human eosinophils and neutrophils. Am J Respir Cell Mol Biol 8:633-639, 1993.

Nolte D., Schmid P., Jager U., Botzlar A., Roesken F., Hecht R., Uhl E., Messmer K. and Vestweber $D$. Leukocyte rolling in venules of striated muscle and skin is mediated by $P$. selectin, not by L-selectin. Am J Physiol 267:H1637-H1642, 1994.

Norman K.E., Moore K.L., McEver R.P. and Ley K. Leukocyte rolling in vivo is mediated by P. selectin glycoprotein ligand-1. Blood 86:4417-4421, 1995.

Nourshargh S., Larkin S.W., Das A. and Williams T.J. Interleukin-1-induced leukocyte extravasation across rat mesenteric microvessels is mediated by platelet-activating factor. Blood 85:2553-2558, 1995.

Olofsson A.M., Arfors K.E., Ramezani L., Wolitzky B.A., Butcher E.C. and von Andrian U.H. Eselectin mediates leukocyte rolling in interleukin-1-treated rabbit mesentery venules. Blood 84:2749-2758, 1994.

oude EgbrinkM.G.A., Tangelder G.J., Slaaf D.W. and Reneman R.S. Thromboembolic reaction following wall puncture in arterioles and venules of the rabbit mesentery. Thromb Haemost 59:23-28, 1988

oude Egbrink M.G.A. Thromboembolic reaction following vessel wall injury in arterioles and venules. Thesis. Maastricht University, 1989.

oude Egbrink M.G.A., Tangelder G.J., Slaaf D.W. and Reneman R.S. Influence of plateletvessel wall interactions on leukocyte rolling in vivo. Circ Res 70:355-363, 1992.

oude Egbrink M.G.A., Tangelder G.J., Slaaf D.W. and Reneman R.S. Different roles of prostaglandins in thromboembolic processes in arterioles and venules in vivo. Thromb Haemost 70:826-833, 1993.

Pacher R., Stanek B., Hulsmann M. and Sinzinger H. Effect of prostaglandin E1 infusion in severe chronic heart failure. Prostaglandins 53:221-235, 1997.

Parmentier S., McGregor L., Catimel B., Leung L.L. and McGregor J.L. Inhibition of platelet functions by a monoclonal antibody (LYP20) directed against a granule membrane glycoprotein (GMP-140/PADGEM). Blood 77:1734-1739, 1991.

Patel K.D., Zimmerman G.A., Prescott S.M., McEver R.P. and McIntyre T.M. Oxygen radicals induce human endothelial cells to express GMP-140 and bind neutrophils. J Cell Biol 112:749-759, 1991.

Phillips M.L., Nudelman E., Gaeta F.C., Perez M., Singhal A.K., Hakomori S. and Paulson J.C. 
ELAM-1 mediates cell adhesion by recognition of a carbohydrate ligand, sialyl-Lex. Science 250:1130-1132, 1990.

Piali L., Fichtel A., Terpe H.J., Imhof B.A. and Gisler R.H. Endothelial vascular cell adhesion molecule 1 expression is suppressed by melanoma and carcinoma. J Exp Med 181:811-816, 1995.

Picker L.J., Warnock R.A., Burns A.R., Doerschuk C.M., Berg E.L. and Butcher E.C. The neutrophil selectin LECAM-1 presents carbohydrate ligands to the vascular selectins ELAM1 and GMP-140. Cell 66:921-933, 1991.

Pober J.S. and Cotran R.S. Cytokines and endothelial cell biology. Physiol Rev 70:427-451, 1990.

Pries A.R., Secomb T.W. and Gaehtgens P. Structure and hemodynamics of microvascular networks: heterogeneity and correlations. Am J Physiol 269:H1713-H1722, 1995.

Raud J., Dahlen S.E., Sydbom A., Lindbom L. and Hedqvist P. Enhancement of acute allergic inflammation by indomethacin is reversed by prostaglandin E2: apparent correlation with in vivo modulation of mediator release. Proc Natl Acad Sci U S A 85:2315-2319, 1988.

Raud J. Intravital microscopic studies on acute mast cell-dependent inflammation. Acta Physiol Scand Suppl 578:1-58, 1989.

Raud J., Dahlen S.E., Smedegard G. and Hedqvist P. An intravital microscopic model for mast cell-dependent inflammation in the hamster cheek pouch. Acta Physiol Scand 135:95-105, 1989a.

Raud J., Lindbom L., Dahlen S.E. and Hedqvist P. Periarteriolar localization of mast cells promotes oriented interstitial migration of leukocytes in the hamster cheek pouch. Am J Pathol 134:161-169, 1989b.

Reneman R.S., Woldhuis B., oude Egbrink M.G.A., Slaaf D.W. and Tangelder G.J. Concentration and velocity profiles of blood cells in the microcirculation. In: Hwang NHC, Turitto VT, Yen MRT, ed. Advances in cardiovascular engineering. New York/London, Plenum Press, 1992: 25-40.

Revtyak G.E., Johnson A.R. and Campbell W.B. Prostaglandin synthesis in bovine coronary endothelial cells: comparison with other commonly studied endothelial cells. Thromb Res 48:671-683, 1987.

Ringler D.H. and Dabich L. Hematology and clinical biochemistry. In: Baker HJ, Lindsey JR, Weisbroth SH, ed. The laboratory rat. New York, Academic Press, 1979: 105-121.

Ritter J.M., Barrow S.E., Blair I.A. and Dollery C.T. Release of prostacyclin in vivo and its role in man. Lancet 1:317-319, 1983.

Roberts W.G. and Palade G.E. Increased microvascular permeability and endothelial fenestration induced by vascular endothelial growth factor. J Cell Sci 108:2369-2379, 1995.

Roitt I.M. Roitt's essential immunology. (9 ed.) Milan, Italy, Blackwell Science, 1997

Rosen S.D. Robert Feulgen Lecture 1993. L-selectin and its biological ligands. Histochemistry 100:185-191, 1993.

Rugh R. The mouse. Its reproduction and development. Oxford, Oxford University Press, 1991

Saito H., Matsuo M., Naminohira S., Sakai T., Kitagawa H. and Hirata A.A. Effect of N-formyl-Lmethionyl-L-leucyl-L-phenylalanine and its analogues on blood pressure. J Pharmacobiodyn 6:141-144, 1983.

Scalia R., Booth G. and Lefer D.J. Vascular endothelial growth factor attenuates leukocyteendothelium interaction during acute endothelial dysfunction: essential role of endothelium derived nitric oxide. FASEB J 13:1039-1046, 1999.

Scheeren T. and Radermacher P. Prostacyclin (PGI2): new aspects of an old substance in the treatment of critically ill patients. Intensive Care Med 23:146-158, 1997.

Schmid-Schönbein G.W., Usami S., Skalak R. and Chien S. The interaction of leukocytes and erythrocytes in capillary and postcapillary vessels. Microvasc Res 19:45-70, 1980.

Schrör K. Prostaglandins, other eicosanoids and endothelial cells. Basic Res Cardiol 80:502$514,1985$.

Siegel B.S. Peace, love and healing. New York, Harper and Row Publishers, 1989 
Siegel S. and Castellan N.J. Nonparametric statistics for the behavioral sciences. ( 2 ed.) Singapore, McGraw-Hill, Inc., 1988

Simpson P.J., Mickelson J., Fantone J.C., Gallagher K.P. and Lucchesi B.R. Iloprost inhibits neutrophil function in vitro and in vivo and limits experimental infarct size in canine heart. Circ Res 60:666-673, 1987.

Simpson P.J., Fantone J.C., Mickelson J.K., Gallagher K.P. and Lucchesi B.R. Identification of a time window for therapy to reduce experimental canine myocardial injury: suppression of neutrophil activation during 72 hours of reperfusion. Circ Res 63:1070-1079, 1988a.

Simpson P.J., Mickelson J., Fantone J.C., Gallagher K.P. and Lucchesi B.R. Reduction of experimental canine myocardial infarct size with prostaglandin E1: inhibition of neutrophil migration and activation. J Pharmacol Exp Ther 244:619-624, $1988 \mathrm{~b}$.

Slaaf D.W., Rood J.P., Tangelder G.J., Jeurens T.J., Alewijnse R., Reneman R.S. and Arts T. A bidirectional optical (BDO) three-stage prism grating system for on-line measurement of red blood cells velocity in microvessels. Microvasc Res 22:110-122, 1981.

Slaaf D.W., Alewijnse R. and Wayland H. Use of telescopic imaging in intravital microscopy: a simple solution for conventional microscopes. Int J Microcirc Clin Exp 1:121-134, 1982.

Slaaf D.W., Arts T., Jeurens T.J.M., Tangelder G.J. and Reneman R.S. Electronic measurement of red blood cell velocity and volume flow in microvessels. In: Chayen J. and Bitensky L., ed. Investigative microtechniques in medicine and biology. New York Basel, Marcel Dekker, Inc., 1984: 327-364.

Slaaf D.W., Jongsma F.H.M., Tangelder G.J. and Reneman R.S. Characteristics of optical systems for intravital microscopy. In: Baker $\mathrm{CH}$, Nastuk WL, ed. Microcirculatory technology. Orlando, Academic Press, Inc., 1986: 211-228.

Slaaf D.W., Tangelder G.J. and Reneman R.S. Physics of the microcirculation. In: Strackee J, Westerhof N, ed. The physics of heart and circulation. Bristol, Institute of Physics Publishing, 1993: $383-416$.

Smith C.W. Endothelial adhesion molecules and their role in inflammation. Can J Physiol Pharmacol 71:76-87, 1993.

Spertini O., Luscinskas F.W., Kansas G.S., Munro J.M., Griffin J.D., Gimbrone M., Jr. and Tedder T.F. Leukocyte adhesion molecule-1 (LAM-1, L-selectin) interacts with an inducible endothelial cell ligand to support leukocyte adhesion. J Immunol 147:2565-2573, 1991.

Springer T.A. Traffic signals for lymphocyte recirculation and leukocyte emigration: the multistep paradigm. Cell 76:301-314, 1994.

Sprugel K.H., McPherson J.M., Clowes A.W. and Ross R. Effects of growth factors in vivo. I. Cell ingrowth into porous subcutaneous chambers. Am J Pathol 129:601-613, 1987.

Srinivasan B.D. and Kulkarni P.S. The role of arachidonic acid metabolites in the mediation of the polymorphonuclear leukocyte response following corneal injury. Invest Ophthalmol Vis Sci 19:1087-1093, 1980.

Staben $\mathrm{P}$. and Albring M. Treatment of patients with peripheral arterial occlusive disease Fontaine stage III and IV with intravenous iloprost: an open study in 900 patients. Prostaglandins Leukot Essent Fatty Acids 54:327-333, 1996.

Steeber D.A., Campbell M.A., Basit A., Ley K. and Tedder T.F. Optimal selectin-mediated rolling of leukocytes during inflammation in vivo requires intercellular adhesion molecule-1 expression. Proc Natl Acad Sci U S A 95:7562-7567, 1998.

Steegmaier M., Levinovitz A., Isenmann S., Borges E., Lenter M., Kocher H.P., Kleuser B. and Vestweber D. The E-selectin-ligand ESL-1 is a variant of a receptor for fibroblast growth factor. Nature 373:615-620, 1995.

Stenberg P.E., McEver R.P., Shuman M.A., Jacques Y.V. and Bainton D.F. A platelet alphagranule membrane protein (GMP-140) is expressed on the plasma membrane after activation. J Cell Biol 101:880-886, 1985.

Subramaniam M., Koedam J.A. and Wagner D.D. Divergent fates of P-and E-selectins after their expression on the plasma membrane. Mol Biol Cell 4:791-801, 1993.

Swerlick R.A. and Lawley T.J. Role of microvascular endothelial cells in inflammation. J Invest 
Dermatol 100:111S-115S, 1993.

Tangelder G.J., Slaaf D.W., Muijtjens A.M., Arts T., oude Egbrink M.G.A. and Reneman R.S. Velocity profiles of blood platelets and red blood cells flowing in arterioles of the rabbit mesentery. Circ Res 59:505-514, 1986.

Tangelder G.J., Slaaf D.W., Arts T. and Reneman R.S. Wall shear rate in arterioles in vivo: least estimates form platelet velocity profiles. Am J Physiol 254:H-1059-H1064, 1988.

Tangelder G.J. and Arfors K.E. Inhibition of leukocyte rolling in venules by protamine and sulfated polysaccharides. Blood 77:1565-1571, 1991.

Tangelder G.J., oude Egbrink M.G.A., Arfors K.-E., Slaaf D.W. and Reneman R.S. Interactions between marginated granulocytes, platelets and venular endothelium in vivo. In: Boccalon H, ed. Vascular Medicine. Amsterdam, Excerpta Medica, 1993: 353-357.

Tangelder G.J., Janssens C.J., Slaaf D.W., oude Egbrink M.G.A. and Reneman R.S. In vivo differentiation of leukocytes rolling in mesenteric postcapillary venules. Am J Physiol 268:H909-H915, 1995.

Taoka Y., Okajima K., Uchiba M., Murakami K., Harada N., Johno M., Naruo M., Okabe H. and Takatsuki K. Reduction of spinal cord injury by administration of iloprost, a stable prostacyclin analog. J Neurosurg 86:1007-1011, 1997.

Tas J. and Geenen L.H. Microspectrophotometric detection of heparin in mast cells and basophilic granulocytes stained metachromatically with Toluidine Blue $\mathrm{O}$. Histochem $\mathrm{J}$ 7:231-248, 1975 .

Tedder T.F., Steeber D.A., Chen A. and Engel P. The selectins: vascular adhesion molecules. Faseb J 9:866-873, 1995.

Thomson I.A., Egginton S., Hudlicka O. and Sims M.H. Iloprost reduces leukocyte adhesion in skeletal muscle venules following ischaemia in a rat model of femorodistal bypass. Eur $\mathrm{J}$ Vasc Surg 8:335-341, 1994.

Thorlacius H., Raud J., Rosengren-Beezley S., Forrest M.J., Hedqvist P. and Lindbom L. Mast cell activation induces $\mathrm{P}$-selectin-dependent leukocyte rolling and adhesion in postcapillary venules in vivo. Biochem Biophys Res Commun 203:1043-1049, 1994.

Thorlacius H., Lindbom L. and Raud J. Cytokine-induced leukocyte rolling in mouse cremaster muscle arterioles in P-selectin dependent. Am J Physiol 272:H1725-H1729, 1997.

Tromp S., Slaaf D., van Breda E., Tangelder G., Reneman R., Rouwet E. and oude Egbrink M. Are mast cells involved in regulation of leukocyte rolling in mesenteric venules of anesthetized rabbits? In: Messmer $K$, Kuebler WM, ed. Sixth world congress for microcirculation. Bologna, Monduzzi Editore, 1996: 649-653.

Tromp S.C., Tangelder G.J., Slaaf D.W., Reneman R.S., van Velzen S., Engels W., van Breda E. and oude Egbrink M.G. The role of mast cells and histamine in leukocyte-endothelium interactions in four rat strains. Pflügers Arch 436:255-261, 1998.

U.K. S.L.I.S.G. Treatment of limb threatening ischaemia with intravenous iloprost: a randomised double-blind placebo controlled study. Eur J Vasc Surg 5:511-516, 1991.

Underwood J.C.E. Lymphoreticular infiltration in human tumors: prognostic and biological implications: a review. Br J Cancer 30:538-548, 1974.

Van Arman C.G. White cells in inflammation. Springfield, Charles C. Thomas, 1974

van Haaster C.M., Derhaag J.G., Engels W., Lemmens P.J., Gijsen A.P., Hornstra G., van der Vusse G.J. and Duijvestijn A.M. Mast cell-mediated induction of ICAM-1, VCAM-1 and Eselectin in endothelial cells in vitro: constitutive release of inducing mediators but no effect of degranulation. Pflugers Arch 435:137-144, 1997.

van Zutphen L.F.M., Baumans V. and Beynen A.C. Proefdieren en dierproeven. Utrecht, Wetenschappelijke uitgeverij Bunge, 1995

Vestweber D. and Blanks J.E. Mechanisms that regulate the function of the selectins and their ligands. Physiol Rev 79:181-213, 1999.

von Andrian U.H., Chambers J.D., McEvoy L.M., Bargatze R.F., Arfors K.E. and Butcher E.C. Two-step model of leukocyte-endothelial cell interaction in inflammation: distinct roles for LECAM-1 and the leukocyte beta 2 integrins in vivo. Proc Natl Acad Sci U S A 88:7538- 
7542, 1991.

von Andrian U.H., Hansell P., Chambers J.D., Berger E.M., Torres-Filho I., Butcher E.C. and Arfors K.E. L-selectin function is required for beta 2-integrin-mediated neutrophil adhesion at physiological shear rates in vivo. Am J Physiol 263:H1034-H1044, 1992.

von Andrian U.H. and Arfors K.E. Neutrophil-endothelial cell interactions in vivo; a chain of events characterized by distinct molecular mechanisms. Agents Actions Suppl 41:153-164, 1993.

von Andrian U.H., Berger E.M., Ramezani L., Chambers J.D., Ochs H.D., Harlan J.M., Paulson J.C., Etzioni A. and Arfors K.E. In vivo behavior of neutrophils from two patients with distinct inherited leukocyte adhesion deficiency syndromes. J Clin Invest 91:2893-2897, 1993a. von Andrian U.H., Chambers J.D., Berg E.L., Michie S.A., Brown D.A., Karolak D., Ramezani L., Berger E.M., Arfors K.E. and Butcher E.C. L-selectin mediates neutrophil rolling in inflamed venules through sialyl LewisX-dependent and -independent recognition pathways. Blood 82:182-191, 1993b.

von Andrian U.H., Hasslen S.R., Nelson R.D., Erlandsen S.L. and Butcher E.C. A central role for microvillous receptor presentation in leukocyte adhesion under flow. Cell 82:989-999, 1995.

Wershil B.K., Wang Z.S., Gordon J.R. and Galli S.J. Recruitment of neutrophils during IgEdependent cutaneous late phase reactions in the mouse is mast cell-dependent. Partial inhibition of the reaction with antiserum against tumor necrosis factor-alpha. J Clin Invest 87:446-453, 1991.

Wigley F.M., Wise R.A., Seibold J.R., McCloskey D.A., Kujala G., Medsger T.A., Jr., Steen V.D., Varga J., Jimenez S., Mayes M. and et al. Intravenous iloprost infusion in patients with Raynaud phenomenon secondary to systemic sclerosis. A multicenter, placebo-controlled, double-blind study. Ann Intern Med 120:199-206, 1994.

Winkler M., Kemp B., Hauptmann S. and Rath W. Parturition: steroids, prostaglandin E2, and expression of adhesion molecules by endothelial cells. Obstet Gynecol 89:398-402, 1997. Wu N.Z., Klitzman B., Dodge R. and Dewhirst M.W. Diminished leukocyte-endothelium interaction in tumor microvessels. Cancer Res 52:4265-4268, 1992.

Wu N.Z., Ross B.A., Gulledge C., Klitzman B., Dodge R. and Dewhirst M.W. Differences in leucocyte-endothelium interactions between normal and adenocarcinoma bearing tissues in response to radiation. $\mathrm{Br} \mathrm{J}$ Cancer 69:883-889, 1994.

Yamaki K., Lindbom L., Thorlacius H., Hedquist P. and Raud J. An approach for studies of mediator-induced leukocyte rolling in the undisturbed microcirculation of the rat mesentery. Br J Pharmacol 123:381-389, 1998.

Yeo E.L., Sheppard J.A. and Feuerstein I.A. Role of P-selectin and leukocyte activation in polymorphonuclear cell adhesion to surface adherent activated platelets under physiologic shear conditions (an injury vessel wall model). Blood 83:2498-2507, 1994.

Young P.E., Baumhueter S. and Lasky L.A. The sialomucin CD34 is expressed on hematopoietic cells and blood vessels during murine development. Blood 85:96-105, 1995.

Zigmond S.H. Mechanisms of sensing chemical gradients by polymorphonuclear leukocytes. Nature 249:450-452, 1974.

Zimmerman B.J., Paulson J.C., Arrhenius T.S., Gaeta F.C. and Granger D.N. Thrombin receptor peptide-mediated leukocyte rolling in rat mesenteric venules: roles of P-selectin and sialyl Lewis X. Am J Physiol 267:H1049-H1053, 1994. 



\section{SUMMARY}

Leukocytes play a pivotal role in host defence reactions. To this purpose, they have to travel via the blood to the area in the body where they are needed and, subsequently, they have to leave the circulation. The process of leukocyteendothelium interactions that precedes this extravasation is a multistep cascade; the rolling of leukocytes along the vessel wall and the adhesion of leukocytes to the endothelial cells are the first steps. It was the aim of the present study to investigate ways in which these leukocyte-endothelium interactions can be modulated in vivo, more specifically how they can be induced or inhibited. Induction of leukocyteendothelium interactions is desired when the defence reaction of the body against invading microorganisms or substances is inadequate. Inhibition of leukocyte rolling and/or adhesion may be beneficial when leukocyte infiltration into tissue has to be prevented, for instance in diseases in which leukocytes are pathogenic.

Methods of local administration are used to study the effects of substances that are scarce and/or expensive, or which might have detrimental or toxic effects when administered systemically. To this purpose, substances can be either infused into the local circulation or topically superfused on the tissue of observation. We determined which of these two routes of administration is preferable in studies focussing on leukocyte rolling in rabbit mesenteric venules (chapter 4). A commonly used vehicle, saline, was either infused through a small side-branch of a feeding artery of the mesenteric segment under observation, or superfused on the segment, and the effects of both ways of administration on leukocyte rolling was studied. To our surprise, we observed that local infusion of saline decreased the level of leukocyte rolling and increased leukocyte rolling velocity, whereas superfusion of saline had no such effects. Therefore, in the studies presented in this thesis local superfusion of substances on the mesentery was the preferred route of administration.

In the experimental studies described in the chapters 5 and 6 the role of mast cells in the induction of leukocyte rolling in mesenteric venules was investigated. Mast cells are packed with granules that contain mediators like histamine, which are rapidly released upon cell activation and degranulation. In addition, mast cell release a variety of other, newly synthesized and biologically active mediators upon activation. The cells are present throughout the body, especially in loose connective tissue and skin. Mast cell degranulation and the consequent release of histamine has been suggested to induce leukocyte rolling in mesenteric venules of SpragueDawley rats. We investigated whether this applies to rabbits and four different rat strains. In rabbits, the influences of mast cell stabilization by cromoglycate, of mast cell degranulation by compound $48 / 80$, and of histamine receptor blockade on leukocyte rolling were studied: no effects were found. These findings were not surprising in view of our histological data: hardly any mast cells are present in the rabbit mesentery (chapter 5). Although mast cells are abundaritly present in the mesentery of Brown Norway, Lewis, Sprague-Dawley, and Wistar rats, the mast cell stabilizer cromoglycate did not affect leukocyte rolling in any of these strains, either. 
After mast cell stabilization, topical administration of histamine increased leukocyte rolling in Sprague-Dawley rats only, while leukocyte adhesion was only increased in Brown Norway rats (chapter 6 ). We conclude, therefore, that the role of mast cells in the induction of leukocyte-endothelium interactions, if any, is strain and species dependent.

Reduction of leukocyte rolling has been observed downstream of a thromboembolic reaction in rabbit mesenteric venules. The mechanism behind this reduction is at present unknown. Chapter 7 describes the possible involvement of selectins, i.e., adhesion molecules involved in leukocyte rolling, in this phenomenon. Thus far, 3 selectins have been identified: L-selectins on leukocytes, and P- and Eselectin on endothelial cells. A thromboembolic reaction was induced in rabbit mesenteric venules in the presence of a monoclonal antibody against each of the 3 selectins and the level of leukocyte rolling downstream of the thrombus was compared to that upstream. We showed that P-selectin appears to be the only selectin involved in leukocyte rolling in the first hour after exteriorization of the mesentery (the early time phase), while $\mathrm{E}$ - and L-selectin mediate leukocyte rolling about two hours after exteriorization (the late time phase). In the early time phase the thromboembolism induced reduction in leukocyte rolling downstream of a thrombus appears to be partly due to inhibition of P-selectin and partly due to diminished involvement in leukocyte rolling of adhesion molecules other than selectins. In the later phase the reduction results from inhibition of other adhesion molecules than the selectins studied, although partial downregulation of E- and Lselectin cannot be excluded (chapter 7).

Prostaglandins (PGs) or their chemical analogues have been shown to be beneficial in several vascular diseases. They are thought to exert their influence through several effects: vasodilation, reduction of platelet aggregation and inhibition of leukocyte functions. The reported effects of prostaglandins on leukocyte functions, however, are variable and most of the studies were performed in vitro. In chapter 8 we investigated the in vivo effects of $P G E_{1}, P G E_{2}, P G I_{2}$, and a combination of these prostaglandins on leukocyte rolling and FMLP induced leukocyte adhesion in rabbit mesenteric venules. Neither $\mathrm{PGE}_{1}, \mathrm{PGE}_{2}$, or $\mathrm{PGI}_{2}$, nor the combination of the three reduced leukocyte rolling or FMLP induced leukocyte adhesion. Instead of an inhibiting effect on leukocyte-endothelium interactions, $\mathrm{PGE}_{2}$ appeared to have a stimulating effect on FMLP induced leukocyte adhesion. Overall, these findings suggest that leukocyte related beneficial effects of prostaglandins in various vascular diseases are due to other effects than inhibition of leukocyte rolling and adhesion.

It has been demonstrated previously that in tumor endothelial cells the expression of adhesion molecules, involved in leukocyte-endothelium interactions, is suppressed. In addition, the upregulation of endothelial adhesion molecules in response to inflammatory cytokines in tumors appears to be markedly hampered. Exposure of endothelial cells to tumor derived angiogenic factors may be responsible for these phenomena. Since this knowledge is primarily based on studies performed in vitro we developed a model to selectively study the effects of 
single tumor derived factors on leukocyte-endothelium interactions in vivo (chapter 9). Slow releasing pellets containing either basic fibroblast growth factor (bFGF). vascular endothelial cell growth factor (VEGF), or vehicle were placed in the scrotum of mice. After 3 days, an intrascrotal injection of the cytokine interleukin-1 $\beta$ was given 4 hours before vessels of the cremaster muscle were observed with intravital microscopy. Exposure to either bFGF or VEGF resulted in markedly decreased levels of cytokines leukocyte adhesion. Suppression of leukocyte rolling was not observed. Hence, in vivo angiogenic factors significantly reduce leukocyte adhesion, the final step preceding leukocyte infiltration. This observation may explain how tumors escape from immune surveillance, and may be helpful in the development of new anti-cancer modalities.

In conclusion, the present thesis shows that leukocyte-endothelium interactions can be modulated in vivo by several cells and substances. Knowledge of these modulating effects is of importance for the development of new anti- and proinflammatory drugs. 



\section{SAMENVATTING}

\section{Leukocyt-vaatwand interacties}

Witte bloedcellen (leukocyten) spelen een belangrijke rol bij de afweerreactie van ons lichaam tegen bijvoorbeeld bacterièn, virussen, of tumorcellen. Om deze organismen of cellen te attaqueren moeten de leukocyten zich via het bloed verplaatsen naar de gebieden waar ze nodig zijn. Vervolgens moeten ze de bloedbaan verlaten (diapedese) om in het omliggende weefsel hun taken uit te kunnen oefenen. Aan het proces van diapedese gaat een aantal stappen vooraf. Allereerst komen er bij een ontstekingsreactie stoffen vrij die de binnenste laag vaatwandcellen (endotheelcellen) kunnen stimuleren tot de expressie van bindingseiwitten, de zogenaamde adhesie-molekulen. Als leukocyten in aanraking komen met die adhesie-molekulen kunnen er zwakke bindingen onstaan. Omdat de leukocyten echter door de bloedstroom ook een voorwaartse kracht ervaren, gaan ze rollen: de cellen bewegen zich al rollend voort langs de vaatwand met een snelheid die beduidend lager is dan de snelheid van de bloedstroom zelf. Op deze wijze verblijven de leukocyten gedurende relatief lange tijd in het ontstekingsgebied; zij kunnen daardoor zelf ook geactiveerd worden door stoffen die in dit gebied zijn vrijgekomen. Geactiveerde leukocyten gaan zich vervolgens stevig hechten aan de endotheelcellen en bewegen zich niet meer voort; dit wordt adhesie genoemd. $\mathrm{Na}$ de adhesie volgt de diapedese: de leukocyten bewegen zich door de vaatwand heen en verplaatsen zich in het omliggende weefsel naar de plek met de hoogste concentratie van onstekingsfactoren, waar ze hun uiteindelijke taak gaan vervullen.

De adhesie-molekulen die het rollen van leukocyten mediëren, zijn de selectines. We onderscheiden drie selectines: L-, P-en E-selectine. L-selectine is aanwezig op de Leukocyten, maar kan worden losgelaten als de cellen geactiveerd worden. $\mathrm{P}$ selectine zit normaliter in bloedPlaatjes en endotheelcellen opgeslagen in granules. $\mathrm{Na}$ activatie van een cel kunnen deze granules binnen minuten fuseren met de celwand, waardoor het P-selectine op het buitenoppervlak van de cel tot expressie komt. E-selectine wordt aangemaakt door geactiveerde Endotheeicellen. De maximale expressie van E-selectine wordt pas 2 tot 6 uur na activatie bereikt.

De adhesie-molekulen die een rol spelen bij de adhesie van leukocyten aan de vaatwand zijn enerzijds integrines op de leukocyten en anderzijds leden van de immunoglobuline superfamilie op het endotheel. Alhoewel de integrines (zoals LFA1 en Mac-1) op ongeactiveerde leukocyten aanwezig zijn, neemt hun bindingscapaciteit sterk toe na activatie van de cel. De integrines kunnen dan met hoge affiniteit binden aan leden van de immunoglobuline superfamilie, bijvoorbeeld ICAM-1 en ICAM-2, op het endotheel. De concentratie van deze adhesie-molekulen neemt sterk toe na activatie van het endotheel.

Leukocyt-vaatwand interacties vinden voornamelijk plaats in de afvoerende, kleine bloedvaten (venulen). Het rollen en de adhesie van leukocyten in venulen kan geobserveerd worden in levende organismen (in vivo) door middel van een intravitaal microscoop. In dit proefschrift worden studies beschreven, waarin leukocyt-vaatwand interacties worden bestudeerd in het darmvlies (mesenterium) 
van verdoofde konijnen en ratten en in de balzakspier (cremasterspier) van verdoofde muizen. Deze weefsels zijn dun en doorzichtig en zijn daardoor zeer geschikt om processen, die zich afspelen in bloedvaten, microscopisch te bestuderen.

\section{Doel van het proefschrift}

Het doel van dit proefschrift was te bestuderen hoe leukocyt-vaatwand interacties in vivo gemoduleerd kunnen worden, en meer specifiek hoe ze kunnen worden geïnduceerd of geïnhibeerd. Inductie van leukocyt-vaatwand interacties is namelijk gewenst als de afweerreactie van het lichaam niet adequaat verloopt. Inhibitie van het rollen of de adhesie van leukocyten is gewenst als de infiltratie van leukocyten in weefsel voorkomen moet worden, bijvoorbeeld in ziektes waarbij leukocyten een belangrijke pathogenetische rol spelen.

\section{Methoden van toediening stoffen}

Als de effecten bestudeerd worden van stoffen die duur of zeldzaam zijn, of die schadelijk zijn als ze in het hele lichaam terecht komen, worden die stoffen vaak lokaal toegediend. Hiertoe kunnen dergelijke stoffen geïnfundeerd worden in bloedvaten in of in de buurt van het geobserveerde weefsel of kunnen ze direct op het weefsel gedruppeld worden (superfusie). Wij hebben bestudeerd welke van deze twee lokale toedieningsmethodes te prefereren is tijdens studies naar leukocytvaatwand interacties (hoofdstuk 4). Een vaak gebruikt oplosmiddel, fysiologisch zout, werd óf geïnfundeerd door een kleine zijtak van een arterie die het te observeren weefsel van bloed voorzag, óf op het weefsel gesuperfundeerd; de effecten van beide toedieningsvormen op het rollen van leukocyten werd bestudeerd. Tot onze verrassing vonden we dat het aantal rollende leukocyten verminderde na infusie van fysiologisch zout en dat de snelheid van de rollende leukocyten toenam, terwijl superfusie van fysiologisch zout geen effect had. Vanwege deze resultaten hebben we besloten om in de rest van de studies in dit proefschrift stoffen lokaal toe te dienen via superfusie op het te bestuderen weefsel.

\section{Mestcellen}

In de experimentele studies die beschreven zijn in de hoofdstukken 5 en 6 is de rol van mestcellen in de inductie van het rollen van leukocyten in venulen van het mesenterium bestudeerd. Mestcellen zitten vol met granules die verschillende mediatoren bevatten, zoals bijvoorbeeld histamine. Activatie van mestcellen kan leiden tot het vrijkomen van de inhoud van de granules (degranulatie). Mestcellen zijn in het hele lichaam aanwezig, vooral in losmazig bindweefsel en in de huid. Er is gesuggereerd dat de degranulatie van mestcellen, en met name het daardoor vrijkomende histamine, het rollen van leukocyten in mesenteriale venulen stimuleert in Sprague-Dawley ratten. We hebben onderzocht of dit ook zo werkt in konijnen en vier verschillende rattenstammen. In konijnen werden de effecten van mestcel stabilisatie door cromoglycaat, van mestcel degranulatie door compound $48 / 80$, en van histamine receptor blokkade bestudeerd: het rollen van de leukocyten werd niet 
beînvloed. Deze bevindingen waren echter niet verwonderlijk in het licht van de histologische data: er zijn nauwelijks mestcellen aanwezig in het mesenterium van konijnen (hoofdstuk 5). Hoewel er wel veel mestcellen aanwezig zijn in het mesenterium van Brown Norway, Lewis, Sprague-Dawley en Wistar ratten, werd het rollen van leukocyten in deze dieren ook niet beïnvloed door de mestcel stabilisator cromoglycaat. Daarop volgende superfusie van het weefsel met histamine stimuleerde het rollen van leukocyten alleen maar in Sprague-Dawley ratten, terwijl de adhesie van leukocyten alleen in Brown Norway ratten werd gestimuleerd (hoofdstuk 6). Deze resultaten betekenen dat, als het al aanwezig is, de rol van mestcellen in het induceren van leukocyt-vaatwand interacties afhankelijk is van de diersoort en de stam die bestudeerd worden.

\section{Thrombo-embolische reactie}

Een interactie tussen geactiveerde bloedplaatjes en een beschadigde vaatwand, een zogenaamde thrombo-embolische reactie, blijkt van invloed te zijn op leukocytvaatwand interacties: stroomafwaarts van een venulaire thrombus is er sprake van een reductie in het rollen van leukocyten. In hoofdstuk 7 wordt de mogelijke betrokkenheid van de verschillende selectines, adhesie molekulen betrokken bij het rollen van leukocyten (zie boven), bij dit fenomeen bestudeerd. In venulen van het mesenterium van het konijn werd een thrombo-embolische reactie geïnduceerd door de vaatwand aan te prikken met een glazen micropipet met een tipdiameter van circa $6 \mu \mathrm{m}$. Op de plek van de beschadiging werd snel een prop van bloedplaatjes gevormd (thrombus), die vervolgens meestal langzaam doorgroeide aan stroomafwaartse zijde; van tijd tot tijd brak zo'n nieuw gevormd stuk van de thrombus af (embolisatie). Deze thrombo-embolische reactie werd geïnduceerd in aanwezigheid van monoclonale antilichamen tegen elk van de drie selectines. Vervolgens werd het aantal rollende leukocyten stroomafwaarts van de thrombus vergeleken met het aantal stroomopwaarts. Uit ons onderzoek bleek dat P-selectine de enige selectine is die betrokken is bij het rollen van leukocyten in het eerste uur van observatie (vroege fase), terwijl E- en L-selectine het rollen van leukocyten mediëren in het tweede uur (late fase). De daling in het rollen van leukocyten, stroomafwaarts van de thrombus, bleek in de vroege fase deels het gevolg te zijn van een verminderde beschikbaarheid en/of functie van P-selectine en deels van een verminderde betrokkenheid van adhesie-molekulen die niet tot de selectine familie behoren. In de late fase bleek de daling voornamelijk voort te komen uit inhibitie van andere adhesie molekulen dan P-, E- en L-selectin, alhoewel een gedeeltelijke remming van $\mathrm{E}$ - en $\mathrm{L}$-selectine niet uitgesloten kan worden.

\section{Prostaglandines}

Prostaglandines (PGs) zijn metabolieten van arachidonzuur, een stof die na activatie van een cel kan worden vrijgemaakt uit de celmembraan. In de (micro)circulatie kunnen een groot aantal cellen prostaglandines produceren na stimulatie: vaatwandcellen (endotheelcellen, spiercellen), bloedcellen (leukocyten en bloedplaatjes) en cellen in het weefsel rondom de vaten (mestcellen). $\mathrm{Er}$ is 
aangetoond dat prostaglandines en hun chemische analogen een gunstig effect hebben in verschillende vaatziekten. Dit positieve effect zou het gevolg kunnen zijn van verschillende activiteiten: inductie van vaatverwijding, remming van aggregatie van bloedplaatjes en remming van de functies van leukocyten. De beschreven effecten van prostaglandines op de functies van leukocyten zijn echter niet eenduidig en de meeste studies op dit gebied zijn in vitro uitgevoerd. In hoofdstuk 8 hebben we de in vivo effecten van 3 verschillende prostaglandines $\left(\mathrm{PGE}_{1}, \mathrm{PGE}_{2}\right.$, en $\mathrm{PGI}_{2}$ ) afzonderlijk en van een combinatie van deze prostaglandines op leukocytvaatwand interacties bestudeerd in venulen van het mesenterium van het konijn; daarbij lag de nadruk op het rollen van leukocyten en op de door het eiwit FMLP geïnduceerde adhesie van leukocyten. $P \mathrm{PE}_{1}, \mathrm{PGE}_{2}, \mathrm{PGI}_{2}$, noch de combinatie van de drie verminderde het rollen of de FMLP geïnduceerde adhesie van leukocyten. $\mathrm{PGE}_{2}$ bleek zelfs een stimulerend effect op FMLP geïnduceerde leukocyt adhesie te hebben. Deze bevindingen suggereren dat leukocyt gerelateerde, gunstige effecten van prostaglandines in verschillende vaatziekten het gevolg zijn van andere effecten dan het remmen van leukocyt rollen en adhesie.

\section{Angiogene factoren}

Endotheelcellen in bloedvaten in tumoren hebben een lager expressie niveau van adhesie-molekulen, die een rol spelen bij leukocyt-vaatwand interacties, dan niettumor gerelateerde endotheelcellen. Bovendien blijkt de stijging in expressie van endotheliale adhesie-molekulen als reactie op ontstekingsfactoren verminderd te zijn in tumoren. Dit fenomeen zou het gevolg kunnen zijn van blootstelling van deze endotheelcellen aan groeifactoren geproduceerd door tumorcellen. Van groeifactoren die de bloedvat nieuwgroei stimuleren, de zogenaamde angiogene factoren, is inderdaad aangetoond dat ze in vitro de expressie van adhesie molekulen remmen. Wij hebben een model ontwikkeld om de effecten van uit tumoren afkomstige, angiogene factoren op leukocyt-vaatwand interacties te bestuderen in vivo (hoofdstuk 9). Pellets die heel langzaam stoffen uitscheiden werden gevuld met basic fibroblast growth factor (bFGF), vascular endothelial cell growth factor (VEGF), of met het oplosmiddel. Vervolgens werden deze pellets geplaatst in de balzak van muizen. Drie dagen later werd in het scrotum het cytokine interleukine-1 $\beta$ geïnjecteerd, ongeveer 4 uur voordat de vaten van de cremasterspier onder de intravitaal microscoop geobserveerd werden. Blootstelling van het weefsel aan bFGF of VEGF resulteerde in een sterk gedaald niveau van cytokinegeïnduceerde leukocyt adhesie. Het rollen van leukocyten was niet verminderd. Samenvattend tonen deze resultaten aan dat angiogene factoren in vivo een remmend effect hebben op de adhesie van leukocyten, de laatste stap die vooraf gaat aan de infiltratie van leukocyten in het omliggende weefsel. Deze bevinding kan verklaren hoe tumoren als het ware ontsnappen aan het immuunsysteem. Tevens geeft deze bevinding een goed aanknopingspunt voor de ontwikkeling van nieuwe anti-kanker therapieën. 


\section{Conclusie}

Concluderend toont dit proefschrift duidelijk aan dat leukocyt-vaatwand interacties in vivo gemoduleerd kunnen worden door verschillende cellen en substanties. We hebben aangetoond dat een thrombo-embolische reactie het rollen van leukocyten remt door een verminderde beschikbaarheid van P-selectine en/of andere adhesiemolekulen. Daarnaast hebben we laten zien dat angiogene factoren in vivo de adhesie van leukocyten remmen, waarschijnlijk ook door verminderde beschikbaarheid of expressie van adhesie molekulen. Daarentegen hebben mestcellen en prostaglandines, waarvan in de literatuur wordt verondersteld dat ze leukocyt-vaatwand interacties kunnen moduleren, geen effect op het rollen of de adhesie van leukocyten in vivo, in venulen van het mesenterium. Kennis van modulerende effecten van factoren op leukocyt-vaatwand interacties is van belang voor de ontwikkeling van nieuwe ontstekingsremmende of -stimulerende medicijnen. 



\section{LIST OF ABBREVIATIONS}

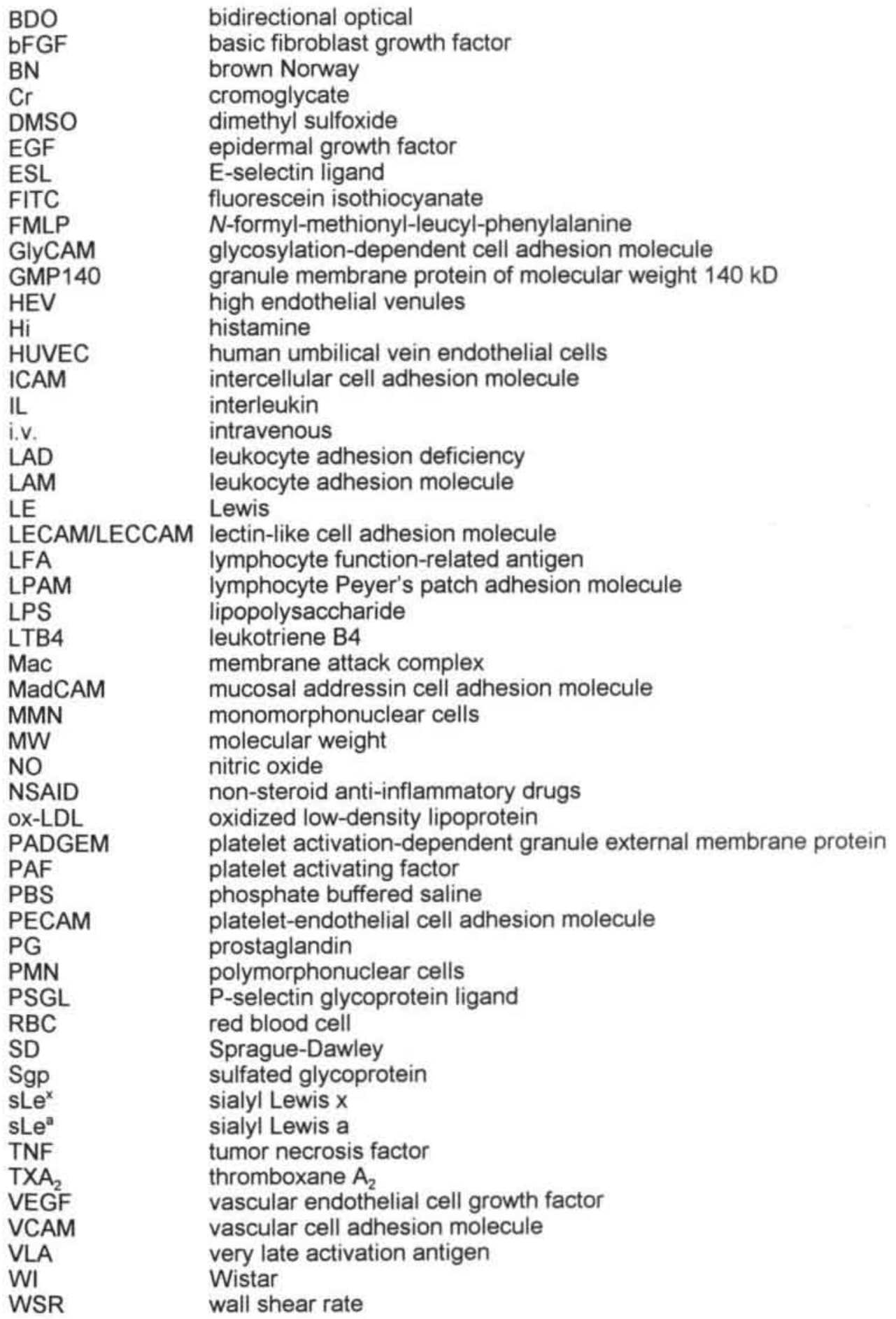





\section{PUBLICATIONS}

\section{Papers:}

M.J.H.A. Kruip, S.C. Tromp, M Verweij and H. Petri. First-time pregnancy, an apparent motive for referral to a Zimbabwean district hospital. Tropical Doctor (1994) 24: 138-139

S.C. Tromp, G.J. Tangelder, D.W. Slaaf, R.S. Reneman, S. van Velzen, W. Engels and M.G.A. oude Egbrink. Mast cells and histamine are not involved in leukocyteendothelium interactions in four rat strains. Pflügers Arch. (1998) 436:255-261

A.W. Griffioen, S.C. Tromp and H.F. Hillen. Angiogenesis modulates the tumour immune response. Int. J. Exp. Pathol. (1998) 79:363-368

S.C. Tromp, G.J. Tangelder, D.W. Slaaf, R.S. Reneman, S van Velzen and M.G.A. oude Egbrink. The influence of prostaglandins on leukocyte-endtohelium interactions in rabbit mesenteric venules. Prostaglandins other lipid mediat. (1999). In press.

M. Egmont-Petersen, U. Schreiner, S.C. Tromp, T. Lehmann, D.W. Slaaf and T. Arts. Detection of leukocytes in contact with the vessel wall from in vivo microscope recordings using a neural network. IEEE Trans. Biomed. Eng. (1999). In press.

\section{Book section:}

S.C. Tromp, D.W. Slaaf, E. van Breda, G.J. Tangelder, R.S. Reneman, E.V. Rouwet and M.G.A. oude Egbrink. Are mast cells involved in regulation of leukocyte rolling in mesenteric venules of anesthetized rabbits? In: 6th World Congress for Microcirculation, ed. K. Messmer and W.M. Kübler, Moduzzi Editore, Bologna (1996) p. 649-653

\section{Abstracts:}

S.C. Tromp, M.G.A. oude Egbrink, R.S. Reneman and G.J. Tangelder. Influence of the stable prostacyclin analogue lloprost on leukocyte rolling in vivo. Pflügers Arch. (1993) 424: R9

S.C. Tromp, M.G.A. oude Egbrink, D.W. Slaaf, R.S. Reneman and G.J. Tangelder Effect of a stable prostacyclin analogue on leukocyte rolling in venules in vivo. Int. J. Microcirc. Clin. Exp. (1993) 13: 49

S.C. Tromp, M.G.A. oude Egbrink, D.W. Slaaf, R.S. Reneman and G.J. Tangelder. The stable prostacyclin analogue lloprost influences number and velocity of rolling leukocytes in mesenteric venules of anesthetized rabbits. Int. J. Microcirc. Clin. Exp. (1994) 14(suppl 1): 60 
S.C. Tromp, M.G.A. oude Egbrink, W. Engels, D.W. Slaaf, R.S. Reneman and G.J. Tangelder. Effect of histamine superfusion following mast cell stabilization on leukocyte rolling in mesenteric venules of three different strains of rats. Pflügers Arch. (1995) 430 (4) [suppl] R95

S.C. Tromp, M.G.A. oude Egbrink, D.W. Slaaf, R.S. Reneman and G.J. Tangelder. lloprost superfusion does not influence leukocyte rolling in rabbit mesenteric venules. Int. J. Microcirc. Clin. Exp. (1995) 15: 107

S.C. Tromp, M.G.A. oude Egbrink, D.W. Slaaf, R.S. Reneman and G.J. Tangelder. Effects of lloprost on leukocyte rolling in rabbit mesenteric venules depend on concentration and way of administration. Int. J. Microcirc. Clin. Exp. (1996) 16: 51

S.C. Tromp, D.W. Slaaf, E. van Breda, G.J. Tangelder, R.S. Reneman and M.G.A. oude Egbrink. Are mast cells involved in regulation of leukocyte rolling in mesenteric venules of anesthetized rabbits? Int. J. Microcirc. Clin. Exp. (1996) 16(suppl 1): 211

S.C. Tromp, G.J. Tangelder, D.W. Slaaf, R.S. Reneman, S. van Velzen, W. Engels and M.G.A. oude Egbrink. Mast cells and leukocyte rolling in four different rat strains. Microcirculation (1997) 4: 130

S.C. Tromp, G.J. Tangeider, D.W. Slaaf, R.S. Reneman, J.S. van Velzen, W. Engels and M.G.A. oude Egbrink. Mast cells and leukocyte rolling in rats and rabbits. Pflügers Arch. (1997) 434: R41

S.C. Tromp, G.J. Tangelder, D.W. Slaaf, R.S. Reneman, J.S. van Velzen, W. Engels and M.G.A. The influence of the prostaglandins E1, E2, and 12 on leukocyte rolling in rabbit mesenteric venules. J. Vasc. Res. (1998) 35:200

S.C. Tromp, D.W. Slaaf, G.J. Tangelder, R.S. Reneman, J.S. van Velzen and M.G.A oude Egbrink. Effects of prostaglandins E1, E2, and I2 on leukocyteendothelium interactions in rabbit mesenteric venules. FASEB J. (1998) 12:A804

S.C. Tromp, M.G.A. oude Egbrink, J.S. van Velzen, D.W. Slaaf, G.J. Tangelder, R.S. Reneman and A.W. Griffioen. Angiogenic factors reduce leukocyte adhesion in mouse cremaster muscle. J. Vasc. Res. (1999) 36:257

S.C. Tromp, M.G.A. oude Egbrink, J.S. van Velzen, D.W. Slaaf, G.J. Tangelder, R.S. Reneman and A.W. Griffioen. Angiogenisis impairs leukocyte adhesion in mouse cremaster muscle. Endothelium (1999) in press 


\section{CURRICULUM VITAE}

Selma Corine Tromp werd geboren op 6 april 1971 te Daarle, gemeente Hellendoorn. $\mathrm{Na}$ het behalen van het VWO diploma aan het Guido de Bres scholengemeenschap te Rotterdam in 1989, begon zij met de studie geneeskunde aan de Rijksuniversiteit Limburg (tegenwoordig: Universiteit Maastricht). Tijdens de doctoraalfase was zij 2 jaar als student-assistent werkzaam op het laboratorium voor microcirculatie van de vakgroep Fysiologie. Voor een verslag van een wetenschapsstage verricht in dit laboratorium kreeg zij een eervolle vermelding van de Faculteit der Geneeskunde. In het derde studiejaar werden drie maanden doorgebracht in een ziekenhuis in Zimbabwe. Het doctoraal diploma werd met genoegen behaald in september 1993 , waarna een aanstelling werd verkregen als AIO bij het Cardiovascular Research Institute Maastricht (CARIM), vakgroep Fysiologie. In december 1997 ontving zij de Jonge Fysiologen aanmoedigingsprijs van de Nederlandse Vereniging voor Fysiologie. Selma combineerde het werk als AIO met de $2 \mathrm{e}$ fase van de studie geneeskunde (coschappen) en behaalde het arts-examen in april 1999. In juli 1999 kwam zij in dienst van het Academisch Ziekenhuis Maastricht als arts-assistent in opleiding tot neuroloog.

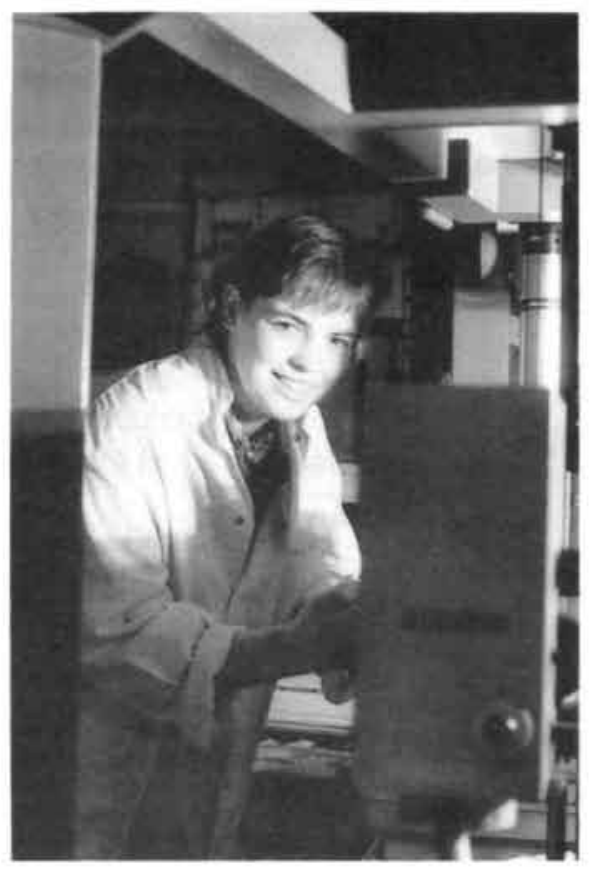

Foto: Franco Gori 



\section{DANKWOORD}

.... when I'm old and wise...people ask me if I knew you

I'll smile and say you were a friend of mine...

Het schrijven van een proefschrift is een heel karwei, maar gelukkig heb ik die klus niet in m'n eentje hoeven klaren. $\mathrm{Er}$ is daarom een aantal mensen dat ik wil bedanken voor de praktische, inhoudelijke en morele steun bij het onderzoek, dat tot het voorliggende proefschrift heeft geleid. Om mijn reputatie een beetje hoog te houden, heb ik een aantal van die bedankjes maar in dichtvorm gegoten.

\section{Rob Reneman}

De man van het overzicht, hooggeleerd

Met vaart als hij iets corrigeert

En wil je een praatje

Dan maakt hij een gaatje

En, Rob, dat wordt ontzettend gewaardeerd

Geert-Jan Tangelder

Een hoogleraar fysiologie uit Amsterdam

Ziet overal het interessante van

Als je het even niet

Meer zo zitten ziet

Bespreek dan je data gewoon met Geert-Jan

\section{Dick Slaaf}

Het lab is ook een fysicus rijk

Recht voor z'n raap, geen gezeik

Zeer zorgvuldig

Niet altijd geduldig

En het ergste is: hij heeft altijd gelijk

\section{Mirjam oude Egbrink}

Bij Mirjam kon ik altijd terecht

En was het schrijven een gevecht

Dan gaf ik het haar

Zij was vaak snel klaar

En had precies de vinger op de zere plek gelegd

\section{Sabrina van Velzen}

Het meisje met de rode haren

Kan ieder dierenklusje klaren

$\mathrm{Er}$ is aan haar

Slechts één bezwaar

Haar mond komt nooit eens tot bedaren 
Naast deze belangrijke mensen zijn er een heleboel anderen geweest die ervoor gezorgd hebben dat ik een leuke tijd heb gehad op het lab. Martijn en ik begonnen tegelijk aan onze AlO-Co-constructie, en hebben zodoende veel cursussen, congressen en dergelijke tegelijkertijd gevolgd. Hij was getuige van één van mijn eerste 'verre' autotochten (helemaal naar Papendal), maar laten we het daar maar niet meer over hebben. Sterkte met de afronding van jouw boekje! Met hem en Berry en Frans heb ik jarenlang met veel plezier de werkkamer gedeeld. Vooral als ik aan het schrijven was, was het prettig een ingewikkelde zinsconstructie met iemand te kunnen delen.

Op het lab was het een komen en gaan van techneuten, AIO's en studenten. In mijn beginperiode op het lab was het vooral Rinus die door maar even ergens naar te wijzen, tegenwerkende apparaten weer op dreef hielp. Eric heeft me regelmatig geholpen door me te laten brainstormen, en Arjan wist me helemaal mee te slepen in zijn enthousiasme over angiogenese. Bij Christine heb ik af en toe mijn hart gelucht, en andersom (het gaat jou ook lukken!). Ellen en Roland (de beukocyten en trompocyten houden we erin) waren er om de boel af en toe op stelten te zetten. Kristel heeft me met name in het laatste jaar veel werk uit handen genomen en ze vond het nog leuk ook. Wat ben ik jaloers geweest op jouw bezoek aan Boston. Miriam en Marijke zorgden er in de laatste maanden voor dat de werkkamer opeens een meidenkamer was geworden, met zeer productieve pieken tussen de belangrijke tennistoernooien en spelletjes in. En dan waren er natuurlijk nog al die mensen van de vakgroep Fysiologie en aanverwanten, die regelmatig even vroegen hoe het ermee was. Bedankt allemaal!

Mijn ouders wil ik bedanken voor hun onvoorwaardelijke steun. Ik kan me wel wat leukers bedenken dan een dochter die een uithoek van Nederland kiest om te gaan studeren en er dan ook nog blijt hangen. Jullie weten, dat ik zelf ook regelmatig even langs zou willen wippen voor een praatje. Gelukkig is de telefoon geduldig. Die heb ik met name ter hand genomen in diepe momenten en tijdens juichstemmingen. Fijn dat jullie er voor me zijn.

De rest van mijn familie en vrienden hebben me regelmatig moe, bleek of hyper aangetroffen. Of dat ooit zal veranderen....? 
Als paranimfen wilde ik graag twee lieve mensen naast me. Een ontzettend lieve meid is Marieke. Tijdens onze vele wandelingen heb je heel wat lief en leed over het onderzoek aangehoord. Het heeft je er gelukkig niet van weerhouden nu ook zelf met een onderzoek bezig te zijn. Ik hoop nog heel wat kilometers met je af te leggen en heel veel koppen thee met je te drinken.

En onbetwist is Woody mijn grote lief. Ons leventje is de laatste tijd wel in een stevige stroomversnelling terecht gekomen. Het spreekwoord huisje, boompje, beestje' zou voor ons herschreven moeten worden in 'huisje, bootje, boekjes'. Ik hoop dat de rust nu een beetje terug gaat keren.

Om nog even bij de termen van het onderzoek te blijven: je kwam als het ware mijn leven binnen rollen en bent gelukkig overgegaan tot adhesie. Laat alsjeblieft maar niet meer los. 


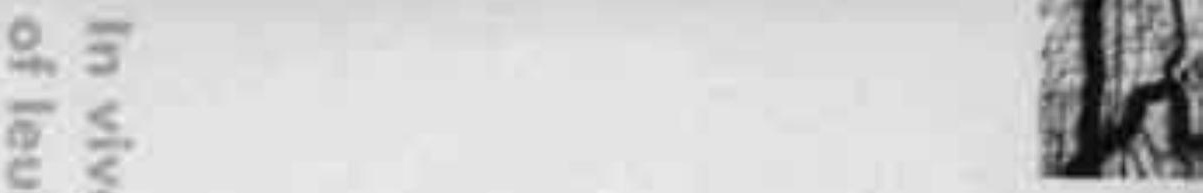

